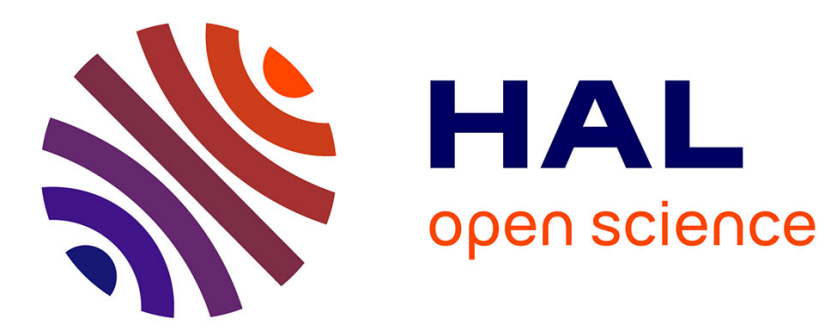

\title{
Prise en charge de la dyspnée chez les patients atteints de BPCO, étude en vraie vie en région Lorraine
}

\author{
Hortense Carette-de Franssu
}

\section{To cite this version:}

Hortense Carette-de Franssu. Prise en charge de la dyspnée chez les patients atteints de BPCO, étude en vraie vie en région Lorraine. Sciences du Vivant [q-bio]. 2017. hal-01945653

\section{HAL Id: hal-01945653 \\ https://hal.univ-lorraine.fr/hal-01945653}

Submitted on 5 Dec 2018

HAL is a multi-disciplinary open access archive for the deposit and dissemination of scientific research documents, whether they are published or not. The documents may come from teaching and research institutions in France or abroad, or from public or private research centers.
L'archive ouverte pluridisciplinaire HAL, est destinée au dépôt et à la diffusion de documents scientifiques de niveau recherche, publiés ou non, émanant des établissements d'enseignement et de recherche français ou étrangers, des laboratoires publics ou privés. 


\section{(1) UNIVERSITÉ}

\section{AVERTISSEMENT}

Ce document est le fruit d'un long travail approuvé par le jury de soutenance et mis à disposition de l'ensemble de la communauté universitaire élargie.

II est soumis à la propriété intellectuelle de l'auteur. Ceci implique une obligation de citation et de référencement lors de l'utilisation de ce document.

D'autre part, toute contrefaçon, plagiat, reproduction illicite encourt une poursuite pénale.

Contact : ddoc-thesesexercice-contact@univ-lorraine.fr

\section{LIENS}

Code de la Propriété Intellectuelle. articles L 122. 4

Code de la Propriété Intellectuelle. articles L 335.2- L 335.10

http://www.cfcopies.com/V2/leg/leg droi.php

http://www.culture.gouv.fr/culture/infos-pratiques/droits/protection.htm 


\section{THÈSE \\ pour obtenir le grade de \\ DOCTEUR EN MÉDECINE}

Présentée et soutenue publiquement

dans le cadre du troisième cycle de Médecine Spécialisée

par

Hortense CARETTE - de FRANSSU

le 2 Octobre 2017

Prise en charge de la dyspnée chez les patients atteints de BPCO, étude en vraie vie en région Lorraine.

Membres du jury :

Président :

M. F.CHABOT

Professeur

Juges :

M. A. CHAOUAT

Professeur

M. B. CHENUEL

Professeur

Mme J.PERRIN

Docteur en Médecine 


\section{(1)}

\section{Président de l'Université de Lorraine : Professeur Pierre MUTZENHARDT}

\section{Doyen de la Faculté de Médecine : Professeur Marc BRAUN}

\section{Vice-doyens}

Pr Karine ANGIOI-DUPREZ, Vice-Doyen

Pr Marc DEBOUVERIE, Vice-Doyen

\begin{abstract}
Assesseurs :
Premier cycle :

Pr Guillaume GAUCHOTTE

Deuxième cycle :

Pr Marie-Reine LOSSER

Troisième cycle :

Pr Marc DEBOUVERIE
\end{abstract}

Innovations pédagogiques : Pr Bruno CHENUEL

Formation à la recherche : Dr Nelly AGRINIER

Affaires juridiques et Relations extérieures : Dr Frédérique CLAUDOT

Vie Facultaire et SIDES : Pr Laure JOLY

Relations Grande Région : Pr Thomas FUCHS-BUDER

\title{
Chargés de mission
}

Bureau de docimologie : Dr Guillaume VOGIN

Commission de prospective facultaire : Pr Pierre-Edouard BOLLAERT

Orthophonie : Pr Cécile PARIETTI-WINKLER

PACES : Dr Mathias POUSSEL

Plan Campus : Pr Bruno LEHEUP

International : Pr Jacques HUBERT

カニーニーニーニーニ

DOYENS HONORAIRES

Professeur Jean-Bernard DUREUX - Professeur Jacques ROLAND - Professeur Patrick NETTER - Professeur Henry COUDANE

\section{PROFESSEURS HONORAIRES}

Etienne ALIOT - Jean-Marie ANDRE - Alain AUBREGE - Gérard BARROCHE Alain BERTRAND - Pierre BEY Marc-André BIGARD - Patrick BOISSEL - Pierre BORDIGONI - Jacques BORRELLY - Michel BOULANGE Jean-Louis BOUTROY - Serge BRIANÇON - Jean-Claude BURDIN - Claude BURLET - Daniel BURNEL Claude CHARDOT Jean-François CHASSAGNE - François CHERRIER Jean-Pierre CRANCE - Gérard DEBRY Emile de LAVERGNE - Jean-Pierre DESCHAMPS - Jean DUHEILLE - Jean-Bernard DUREUX - Gilbert FAURE Gérard FIEVE Bernard FOLIGUET Jean FLOQUET - Robert FRISCH - Alain GAUCHER - Pierre GAUCHER Professeur Jean-Luc GEORGE - Alain GERARD Hubert GERARD - Jean-Marie GILGENKRANTZ - Simone GILGENKRANTZ - Gilles GROSDIDIER - Oliéro GUERCI - Philippe HARTEMANN Gérard HUBERT - Claude HURIET Christian JANOT - Michèle KESSLER - François KOHLER - Jacques LACOSTE - Henri LAMBERT Pierre LANDES - Marie-Claire LAXENAIRE - Michel LAXENAIRE - Alain LE FAOU Jacques LECLERE - Pierre LEDERLIN - Bernard LEGRAS - Jean-Pierre MALLIÉ - Philippe MANGIN - Jean-Claude MARCHAL - Yves MARTINET Pierre MATHIEU - Michel MERLE - Pierre MONIN Pierre NABET - Patrick NETTER - Jean-Pierre NICOLAS - Pierre PAYSANT Francis PENIN - Gilbert PERCEBOIS Claude PERRIN - Luc PICARD - François PLENAT - Jean-Marie POLU - Jacques POUREL - Jean PREVOT - Francis RAPHAEL - Antoine RASPILLER Denis REGENT - Michel RENARD - Jacques ROLAND - Daniel SCHMITT - Michel SCHMITT - Michel SCHWEITZER - Daniel SIBERTIN-BLANC - Claude SIMON Danièle SOMMELET - Jean-François STOLTZ Michel STRICKER - Gilbert THIBAUT - Gérard VAILLANT - Paul VERT - Hervé VESPIGNANI - Colette VIDAILHET - Michel VIDAILHET - Jean-Pierre VILLEMOT - Michel WEBER 


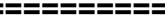

\section{PROFESSEURS ÉMÉRITES}

Professeur Etienne ALIOT - Professeur Gérard BARROCHE - Professeur Serge BRIANÇON - Professeur JeanPierre CRANCE Professeur Gilbert FAURE - Professeur Bernard FOLIGUET - Professeur Alain GERARD Professeur Gilles GROSDIDIER

Professeur Philippe HARTEMANN - Professeur François KOHLER - Professeur Alain LE FAOU - Professeur Jacques LECLERE

Professeur Yves MARTINET - Professeur Patrick NETTER - Professeur Jean-Pierre NICOLAS - Professeur Luc PICARD - Professeur François PLENAT - Professeur Jean-François STOLTZ

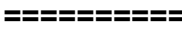

\section{PROFESSEURS DES UNIVERSITÉS - PRATICIENS HOSPITALIERS}

(Disciplines du Conseil National des Universités)

$42^{\text {ème }}$ Section : MORPHOLOGIE ET MORPHOGENĖSE

$1^{\text {ère }}$ sous-section : (Anatomie)

Professeur Marc BRAUN - Professeure Manuela PEREZ

$2^{\text {eme }}$ sous-section : (Histologie, embryologie et cytogénétique)

Professeur Christo CHRISTOV

$3^{\text {eme }}$ sous-section : (Anatomie et cytologie pathologiques)

Professeur Jean-Michel VIGNAUD - Professeur Guillaume GAUCHOTTE

$43^{\text {ème }}$ Section : BIOPHYSIQUE ET IMAGERIE MÉDICALE

$1^{\text {ère }}$ sous-section : (Biophysique et médecine nucléaire)

Professeur Gilles KARCHER - Professeur Pierre-Yves MARIE - Professeur Pierre OLIVIER

$2^{\mathrm{e} m e}$ sous-section : (Radiologie et imagerie médicale)

Professeur René ANXIONNAT - Professeur Alain BLUM - Professeur Serge BRACARD - Professeur Michel CLAUDON Professeure Valérie CROISÉ-LAURENT - Professeur Jacques FELBLINGER - Professeur Pedro GONDIM TEIXEIRA

\author{
$44^{\text {ème }}$ Section : BIOCHIMIE, BIOLOGIE CELLULAIRE ET MOLÉCULAIRE, PHYSIOLOGIE ET \\ NUTRITION \\ $1^{\text {ère }}$ sous-section : (Biochimie et biologie moléculaire) \\ Professeur Jean-Louis GUEANT - Professeur Bernard NAMOUR - Professeur Jean-Luc OLIVIER \\ $2^{\text {ème }}$ sous-section : (Physiologie) \\ Professeur Christian BEYAERT - Professeur Bruno CHENUEL - Professeur François MARCHAL \\ $4^{\text {ème }}$ sous-section : (Nutrition) \\ Professeur Didier QUILLIOT - Professeure Rosa-Maria RODRIGUEZ-GUEANT - Professeur Olivier ZIEGLER
}

\author{
45 $5^{\text {ème }}$ Section : MICROBIOLOGIE, MALADIES TRANSMISSIBLES ET HYGIÈNE \\ $1^{\text {ère }}$ sous-section : (Bactériologie - virologie; hygiène hospitalière) \\ Professeur Alain LOZNIEWSKI - Professeure Evelyne SCHVOERER \\ $2^{\mathrm{e} m e}$ sous-section : (Parasitologie et Mycologie) \\ Professeure Marie MACHOUART \\ $3^{\text {ème }}$ sous-section : (Maladies infectieuses; maladies tropicales) \\ Professeur Thierry MAY - Professeure Céline PULCINI - Professeur Christian RABAUD
}

$46^{\text {ème }}$ Section : SANTÉ PUBLIQUE, ENVIRONNEMENT ET SOCIÉTÉ

$1^{\text {ère }}$ sous-section : (Épidémiologie, économie de la santé et prévention)

Professeur Francis GUILLEMIN - Professeur Denis ZMIROU-NAVIER

$3^{\text {ème }}$ sous-section : (Médecine légale et droit de la santé)

Professeur Henry COUDANE

$4^{\mathrm{e} m e}$ sous-section : (Biostatistiques, informatique médicale et technologies de communication)

Professeure Eliane ALBUISSON - Professeur Nicolas JAY

$47^{\text {ème }}$ Section : CANCÉROLOGIE, GÉNÉTIQUE, HÉMATOLOGIE, IMMUNOLOGIE

$1^{\text {ère }}$ sous-section : (Hématologie; transfusion)

Professeur Pierre FEUGIER

$2^{\mathrm{e}}{ }^{\mathrm{eme}}$ sous-section : (Cancérologie; radiothérapie)

Professeur Thierry CONROY - Professeur François GUILLEMIN - Professeur Didier PEIFFERT - Professeur Frédéric MARCHAL

$3^{\text {ème }}$ sous-section : (Immunologie)

Professeur Marcelo DE CARVALHO-BITTENCOURT - Professeure Marie-Thérèse RUBIO

$4^{\text {ème }}$ sous-section : (Génétique)

Professeur Philippe JONVEAUX - Professeur Bruno LEHEUP 
48 $8^{\text {ème }}$ Section : ANESTHÉSIOLOGIE, RÉANIMATION, MÉDECINE D'URGENCE, PHARMACOLOGIE ET THÉRAPEUTIQUE

$1^{\text {ère }}$ sous-section : (Anesthésiologie-réanimation)

Professeur Gérard AUDIBERT - Professeur Hervé BOUAZIZ - Professeur Thomas FUCHS-BUDER

Professeure Marie-Reine LOSSER - Professeur Claude MEISTELMAN

$2^{\text {ème }}$ sous-section : (Réanimation)

Professeur Pierre-Édouard BOLLAERT - Professeur Sébastien GIBOT - Professeur Bruno LÉVY

$3^{\text {ème }}$ sous-section : (Pharmacologie fondamentale; pharmacologie clinique ; addictologie)

Professeur Pierre GILLET - Professeur Jean-Yves JOUZEAU

$4^{\text {ème }}$ sous-section : (Thérapeutique ; addictologie)

Professeur François PAILLE - Professeur Patrick ROSSIGNOL - Professeur Faiez ZANNAD

$49^{\text {ème }}$ Section : PATHOLOGIE NERVEUSE ET MUSCULAIRE, PATHOLOGIE MENTALE, HANDICAP ET RÉÉDUCATION

$1^{\text {ère }}$ sous-section : (Neurologie)

Professeur Marc DEBOUVERIE - Professeur Louis MAILLARD - Professeur Luc TAILLANDIER - Professeure Louise TYVAERT

$2^{\text {ème }}$ sous-section : (Neurochirurgie)

Professeur Jean AUQUE - Professeur Thierry CIVIT - Professeure Sophie COLNAT-COULBOIS - Professeur Olivier KLEIN

$3^{\text {ème }}$ sous-section : (Psychiatrie d'adultes ; addictologie)

Professeur Jean-Pierre KAHN - Professeur Raymund SCHWAN

$4^{\text {ème }}$ sous-section : (Pédopsychiatrie ; addictologie)

Professeur Bernard KABUTH

$5^{\text {ème }}$ sous-section : (Médecine physique et de réadaptation)

Professeur Jean PAYSANT

$50^{\text {ème }}$ Section : PATHOLOGIE OSTÉO-ARTICULAIRE, DERMATOLOGIE ET CHIRURGIE PLASTIQUE

$1^{\text {ère }}$ sous-section : (Rhumatologie)

Professeure Isabelle CHARY-VALCKENAERE - Professeur Damien LOEUILLE

$2^{\mathrm{e} \text { me }}$ sous-section : (Chirurgie orthopédique et traumatologique)

Professeur Laurent GALOIS - Professeur Didier MAINARD - Professeur Daniel MOLE - Professeur François SIRVEAUX

$3^{\text {eme }}$ sous-section : (Dermato-vénéréologie)

Professeur Jean-Luc SCHMUTZ

$4^{\mathrm{e} m e}$ sous-section : (Chirurgie plastique, reconstructrice et esthétique ; brûlologie)

Professeur François DAP - Professeur Gilles DAUTEL - Professeur Etienne SIMON

$51^{\text {ème }}$ Section : PATHOLOGIE CARDIO-RESPIRATOIRE ET VASCULAIRE

$1^{\text {ère }}$ sous-section : (Pneumologie ; addictologie)

Professeur Jean-François CHABOT - Professeur Ari CHAOUAT

$2^{\text {ème }}$ sous-section : (Cardiologie)

Professeur Edoardo CAMENZIND - Professeur Christian de CHILLOU DE CHURET - Professeur Yves JUILLIERE

Professeur Nicolas SADOUL

$3^{\text {eme }}$ sous-section : (Chirurgie thoracique et cardiovasculaire)

Professeur Thierry FOLLIGUET - Professeur Juan-Pablo MAUREIRA

$4^{\mathrm{e} m e}$ sous-section : (Chirurgie vasculaire; médecine vasculaire)

Professeur Sergueï MALIKOV - Professeur Denis WAHL - Professeur Stéphane ZUILY

$52^{\text {ème }}$ Section : MALADIES DES APPAREILS DIGESTIF ET URINAIRE

$1^{\text {ère }}$ sous-section : (Gastroentérologie ; hépatologie ; addictologie)

Professeur Jean-Pierre BRONOWICKI - Professeur Laurent PEYRIN-BIROULET

$3^{\text {ème }}$ sous-section : (Néphrologie)

Professeur Luc FRIMAT - Professeure Dominique HESTIN

$4{ }^{\text {ème }}$ sous-section : (Urologie)

Professeur Pascal ESCHWEGE - Professeur Jacques HUBERT

$53^{\text {ème }}$ Section : MÉdECINE INTERNE, GÉRIATRIE, CHIRURGIE GÉNÉRALE ET MÉDECINE GÉNÉRALE

$1^{\text {ère }}$ sous-section : (Médecine interne ; gériatrie et biologie du vieillissement ; addictologie)

Professeur Athanase BENETOS - Professeur Jean-Dominique DE KORWIN - Professeure Gisèle KANNY

Professeure Christine PERRET-GUILLAUME - Professeur Roland JAUSSAUD - Professeure Laure JOLY

$2^{\mathrm{e} e \mathrm{e} e}$ sous-section : (Chirurgie générale)

Professeur Ahmet AYAV - Professeur Laurent BRESLER - Professeur Laurent BRUNAUD 
$3^{\text {ème }}$ sous-section : (Médecine générale)

Professeur Jean-Marc BOIVIN - Professeur Paolo DI PATRIZIO

$54^{\text {ème }}$ Section : DÉVELOPPEMENT ET PATHOLOGIE DE L'ENFANT, GYNÉCOLOGIE$1^{\text {ère }}$ sous-section : (Pédiatrie) OBSTÉTRIQUE, ENDOCRINOLOGIE ET REPRODUCTION

Professeur Pascal CHASTAGNER - Professeur François FEILLET - Professeur Jean-Michel HASCOET

Professeur Emmanuel RAFFO - Professeur Cyril SCHWEITZER

$2^{\text {ème }}$ sous-section : (Chirurgie infantile)

Professeur Pierre JOURNEAU - Professeur Jean-Louis LEMELLE

$3^{\text {ème }}$ sous-section : (Gynécologie-obstétrique ; gynécologie médicale)

Professeur Philippe JUDLIN - Professeur Olivier MOREL

$4^{\text {ème }}$ sous-section : (Endocrinologie, diabète et maladies métaboliques; gynécologie médicale)

Professeur Bruno GUERCI - Professeur Marc KLEIN - Professeur Georges WERYHA

$55^{\text {ème }}$ Section : PATHOLOGIE DE LA TÊTE ET DU COU

$1^{\text {ere }}$ sous-section : (Oto-rhino-laryngologie)

Professeur Roger JANKOWSKI - Professeure Cécile PARIETTI-WINKLER

$2^{\mathrm{e} \text { eme }}$ sous-section : (Ophtalmologie)

Professeure Karine ANGIOI - Professeur Jean-Paul BERROD

$3^{\mathrm{e} \text { èe }}$ sous-section : (Chirurgie maxillo-faciale et stomatologie)

Professeure Muriel BRIX

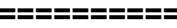

PROFESSEURS DES UNIVERSITÉS

$61^{{ }^{\text {ème }}}$ Section : GÉNIE INFORMATIQUE, AUTOMATIQUE ET TRAITEMENT DU SIGNAL

Professeur Walter BLONDEL

$64^{\text {ème }}$ Section : BIOCHIMIE ET BIOLOGIE MOLÉCULAIRE

Professeure Sandrine BOSCHI-MULLER - Professeur Pascal REBOUL

$65^{\text {ème }}$ Section : BIOLOGIE CELLULAIRE

Professeure Céline HUSELSTEIN

$==$ =ニ=ニ=

PROFESSEUR ASSOCIÉ DE MÉDECINE GÉNÉRALE

Professeur associé Sophie SIEGRIST

$=====+=$

MAÎTRES DE CONFÉRENCES DES UNIVERSITÉS - PRATICIENS HOSPITALIERS

$42^{\mathrm{ème}}$ Section : MORPHOLOGIE ET MORPHOGENÈSE

$1^{\text {ère }}$ sous-section : (Anatomie)

Docteur Bruno GRIGNON

$2^{\mathrm{e} \text { èe }}$ sous-section : (Histologie, embryologie et cytogénétique)

Docteure Chantal KOHLER

$43^{\text {ème }}$ Section : BIOPHYSIQUE ET IMAGERIE MÉDICALE

$1^{\text {ère }}$ sous-section : (Biophysique et médecine nucléaire)

Docteur Antoine VERGER (stagiaire)

$2^{\mathrm{e} m e}$ sous-section : (Radiologie et imagerie médicale)

Docteur Damien MANDRY

$44^{\text {ème }}$ Section : BIOCHIMIE, BIOLOGIE CELLULAIRE ET MOLÉCULAIRE, PHYSIOLOGIE ET NUTRITION

$1^{\text {ère }}$ sous-section : (Biochimie et biologie moléculaire)

Docteure Shyue-Fang BATTAGLIA - Docteure Sophie FREMONT - Docteure Isabelle AIMONE-GASTIN

Docteure Catherine MALAPLATE-ARMAND - Docteur Marc MERTEN - Docteur Abderrahim OUSSALAH

$2^{\text {ème }}$ sous-section : (Physiologie)

Docteure Silvia DEMOULIN-ALEXIKOVA - Docteur Mathias POUSSEL - Docteur Jacques JONAS (stagiaire)

$3^{\text {ème }}$ sous-section : (Biologie Cellulaire)

Docteure Véronique DECOT-MAILLERET 
45 $5^{\text {ème }}$ Section : MICROBIOLOGIE, MALADIES TRANSMISSIBLES ET HYGIÈNE

$1^{\text {ère }}$ sous-section : (Bactériologie - Virologie ; hygiène hospitalière)

Docteure Corentine ALAUZET - Docteure Hélène JEULIN - Docteure Véronique VENARD

$2^{\text {eme }}$ sous-section : (Parasitologie et mycologie)

Docteure Anne DEBOURGOGNE

$46^{\text {ème }}$ Section : SANTÉ PUBLIQUE, ENVIRONNEMENT ET SOCIÉTÉ

$1^{\text {ère }}$ sous-section : (Epidémiologie, économie de la santé et prévention)

Docteure Nelly AGRINIER - Docteur Cédric BAUMANN - Docteure Frédérique CLAUDOT - Docteur Alexis HAUTEMANIËRE

$2^{\text {ème }}$ sous-section (Médecine et Santé au Travail)

Docteure Isabelle THAON

$3^{\text {ème }}$ sous-section (Médecine légale et droit de la santé)

Docteur Laurent MARTRILLE

$47^{\text {ème }}$ Section : CANCÉROLOGIE, GÉNÉTIQUE, HÉMATOLOGIE, IMMUNOLOGIE

$1^{\text {èr }}$ sous-section : (Hématologie ; transfusion)

Docteure Aurore PERROT - Docteur Julien BROSEUS

$2^{\mathrm{e} m e}$ sous-section : (Cancérologie ; radiothérapie)

Docteure Lina BOLOTINE - Docteur Guillaume VOGIN

$4^{\text {ème }}$ sous-section : (Génétique)

Docteure Céline BONNET

$48^{\text {ème }}$ Section : ANESTHÉSIOLOGIE, RÉANIMATION, MÉDECINE D'URGENCE, PHARMACOLOGIE ET THÉRAPEUTIQUE

$2^{\text {ème }}$ sous-section : (Réanimation; Médecine d'urgence)

Docteur Antoine KIMMOUN

$3^{\text {ème }}$ sous-section : (Pharmacologie fondamentale ; pharmacologie clinique ; addictologie)

Docteur Nicolas GAMBIER - Docteure Françoise LAPICQUE - Docteur Julien SCALA-BERTOLA

$4^{\text {eme }}$ sous-section : (Thérapeutique ; Médecine d'urgence ; addictologie)

Docteur Nicolas GIRERD

$50^{\text {ème }}$ Section: PATHOLOGIE OSTÉO-ARTICULAIRE, DERMATOLOGIE ET CHIRURGIE PLASTIQUE

$1^{\text {ere }}$ sous-section : (Rhumatologie)

Docteure Anne-Christine RAT

$3^{\text {ème }}$ sous-section : (Dermato-vénéréologie)

Docteure Anne-Claire BURSZTEJN

$4^{\text {ème }}$ sous-section : (Chirurgie plastique, reconstructrice et esthétique ; brûlologie)

Docteure Laetitia GOFFINET-PLEUTRET

$51^{\text {ème }}$ Section : PATHOLOGIE CARDIO-RESPIRATOIRE ET VASCULAIRE

$3^{\text {eme }}$ sous-section : (Chirurgie thoracique et cardio-vasculaire)

Docteur Fabrice VANHUYSE

$52^{\text {ème }}$ Section : MALADIES DES APPAREILS DIGESTIF ET URINAIRE

$1^{\text {ère }}$ sous-section : (Gastroentérologie ; hépatologie ; addictologie)

Docteur Jean-Baptiste CHEVAUX - Docteur Anthony LOPEZ (stagiaire)

$53^{\text {ème }}$ Section : MÉDECINE INTERNE, GÉRIATRIE, CHIRURGIE GÉNÉRALE ET MÉDECINE GÉNÉRALE

$2^{\text {ème }}$ sous-section : (Chirurgie générale)

Docteur Cyril PERRENOT (stagiaire)

$3^{\text {ème }}$ sous-section : (Médecine générale)

Docteure Elisabeth STEYER

$54^{\text {ème }}$ Section : DEVELOPPEMENT ET PATHOLOGIE DE L'ENFANT, GYNECOLOGIEOBSTETRIQUE, ENDOCRINOLOGIE ET REPRODUCTION

$5^{\text {ème }}$ sous-section : (Biologie et médecine du développement et de la reproduction; gynécologie médicale)

Docteure Isabelle KOSCINSKI

$55^{\text {ème }}$ Section : PATHOLOGIE DE LA TÊTE ET DU COU

$1^{\text {ere }}$ sous-section : (Oto-Rhino-Laryngologie)

Docteur Patrice GALLET 


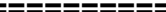

\section{MAITTRES DE CONFÉRENCES}

\section{$5^{\text {ème }}$ Section : SCIENCES ÉCONOMIQUES}

Monsieur Vincent LHUILLIER

$7^{\text {ème }}$ Section : SCIENCES DU LANGAGE : LINGUISTIQUE ET PHONETIQUE GENERALES

Madame Christine DA SILVA-GENEST

$19^{\text {ème }}$ Section : SOCIOLOGIE, DÉMOGRAPHIE

Madame Joëlle KIVITS

$64^{\text {ème }}$ Section : BIOCHIMIE ET BIOLOGIE MOLÉCULAIRE

Madame Marie-Claire LANHERS - Monsieur Nick RAMALANJAONA

\section{$65^{\text {ème }}$ Section : BIOLOGIE CELLULAIRE}

Madame Nathalie AUCHET - Madame Natalia DE ISLA-MARTINEZ - Monsieur Jean-Louis GELLY - Madame Ketsia HESS Monsieur Hervé MEMBRE - Monsieur Christophe NEMOS

$66^{\text {ème }}$ Section : PHYSIOLOGIE

Monsieur Nguyen TRAN

\section{MAÎTRES DE CONFÉRENCES ASSOCIÉS DE MÉDECINE GÉNÉRALE}

Docteur Pascal BOUCHE - Docteur Olivier BOUCHY - Docteur Arnaud MASSON - Docteur Cédric BERBE

Docteur Jean-Michel MARTY

\section{DOCTEURS HONORIS CAUSA}

Professeur Charles A. BERRY (1982)

Centre de Médecine Préventive, Houston (U.S.A)

Professeur Pierre-Marie GALETTI (1982)

Brown University, Providence (U.S.A)

Professeure Mildred T. STAHLMAN (1982)

Vanderbilt University, Nashville (U.S.A)

Professeur Théodore H. SCHIEBLER (1989)

Institut d'Anatomie de Würtzburg (R.F.A)

Université de Pennsylvanie (U.S.A)

Professeur Mashaki KASHIWARA (1996)

Research Institute for Mathematical Sciences de Kyoto (JAPON)
Professeure Maria DELIVORIA- Professeur Brian BURCHELL (2007) PAPADOPOULOS (1996) Professeur Ralph GRÄSBECK (1996) Université d'Helsinki (FINLANDE) Professeur Duong Quang TRUNG (1997)

Université d'Hô Chi Minh-Ville (VIÊTNAM)

Professeur Daniel G. BICHET (2001) Université de Montréal (Canada)

Professeur Marc LEVENSTON (2005) Institute of Technology, Atlanta (USA)
Université de Dundee (Royaume-Uni)

Professeur Yunfeng ZHOU (2009)

Université de Wuhan (CHINE)

Professeur David ALPERS (2011)

Université de Washington (U.S.A)

Professeur Martin EXNER (2012)

Université de Bonn (ALLEMAGNE) 


\section{REMERCIEMENTS}

À NOTRE MAITRE, PRÉSIDENT DE JURY ET DIRECTEUR DE THÈSE,

Monsieur le Professeur François Chabot,

Professeur de Pneumologie

Vous nous faites l'honneur de présider ce jury de thèse. Nous vous remercions vivement pour votre aide précieuse et indispensable à la réalisation de ce travail.

Nous vous remercions de nous avoir offert une formation pneumologique et humaine de grande qualité. Nous nous engageons à faire honneur à votre enseignement rigoureux au cours des années à venir.

Soyez assuré de notre reconnaissance, de notre admiration et de notre profond respect. 


\section{À NOTRE MAITRE ET JUGE}

Monsieur le Professeur Ari Chaouat,

Professeur de Pneumologie

Vous nous faites l'honneur de juger cette thèse.

Vous avez encadré nos premiers pas d'interne au sein du département de Pneumologie avec bienveillance et nous vous remercions particulièrement pour votre patience, vos attentions et votre disponibilité.

Soyez assuré de notre admiration et de notre profond respect. 


\section{À NOTRE MAITRE ET JUGE}

Monsieur le Professeur Bruno Chenuel,

Professeur de Physiologie

Nous vous remercions de l'honneur que vous nous faites en acceptant de juger ce travail.

Nous sommes ravies de bénéficier ce jour de votre expertise.

Veuillez trouver ici le témoignage de notre profond respect et de notre sincère reconnaissance. 


\section{À NOTRE JUGE}

Madame le Docteur Julie Perrin,

Docteur en Pneumologie

Tu nous fais l'honneur de juger cette thèse.

Ce projet est aussi le tien, je te remercie de tout cœur pour ton implication, ta disponibilité, ta confiance et ton aide si précieuse à la réalisation de ce travail.

Tu as grandement participé à ma formation pneumologique et j'apprends toujours beaucoup à tes côtés. Je te remercie pour ta pertinence, ta rigueur et ta sérénité.

Sois assurée de ma profonde reconnaissance et de mon admiration. 
Au Docteur Maéva Zysman, pneumologue, sans qui ce projet n'aurait sans doute pu aboutir tel qu'il est.

Je te remercie de tout cœur pour ton aide si précieuse à la réalisation de ce travail, pour ton investissement à chaque étape de notre projet, pour ta disponibilité à toute épreuve et pour ton regard si pertinent. Je me sais très chanceuse d'avoir bénéficié de tes conseils au cours de cet internat, tant sur le plan clinique que méthodologique. Je profite des ces quelques lignes pour te témoigner ma reconnaissance et ma profonde admiration.

A Monsieur le Professeur Nicolas Roche, Professeur de Pneumologie.

Nous vous remercions vivement de nous avoir donné accès aux données de la cohorte iBPCO nécessaires à la réalisation de ce travail. Nous vous remercions également pour votre confiance et vos précieux conseils. Soyez assuré de notre profond respect et de notre sincère reconnaissance.

A Madame le Docteur Capucine Morélot-Panzini, Docteur en Pneumologie.

Vous nous avez permis d'aller au bout de notre projet grâce à votre questionnaire concernant la dyspnée et nous vous en remercions infiniment. Dans le cadre de cette étude et à l'aide de vos travaux si complets, nous avons pu découvrir et affiner nos connaissances concernant ce concept si complexe et passionnant qu'est la dyspnée. Soyez assuré de notre profond respect et de notre sincère reconnaissance. 
A Madame le Docteur Anne Guillaumot, pneumologue du département de pneumologie du CHU de Nancy et Présidente du Collège Lorrain des Pathologies Thoraciques (CLPT). Nous vous remercions pour votre aide précieuse à la diffusion des questionnaires et pour vos encouragements.

A Brigitte Risse et aux médecins qui ont participé aux inclusions dans la cohorte iBPCO.

Au médecins de Nancy, Verdun, Epinal et Metz qui ont activement participé à ma formation, particulièrement au Professeur Peiffert et aux docteurs Emmanuel Gomez, Ayman Kheir, Julie Chamard, Jean-Christophe Faivre, Anne-Sophie Baumann, Madame Beckendorf, Céline Gavoille, Lionnel Geoffroy, Cyril Abdeddaim, Monsieur Allam, Bernard Champy, Abdessamad Zahdour, Gina Constantin, Chahinaze Ramdame, Madame Arboit, Magalie Mercy, Stéphane Raymond, Pierre Cervantes, Pascale Martin et Benoit Godbert.

Au Professeur Vincent Jounieaux du CHU d'Amiens et à toute son équipe, pour leur accueil et leur confiance, et particulièrement aux docteurs Claire Andréjak, Claire Poulet, Géraldine François et Jean-Baptiste Obstler,

Aux équipes paramédicales et aux kinésithérapeutes.

A tous mes co-internes de pneumologie de Nancy, devenus chefs pour certains, particulièrement à mes très chers Romain et Arnaud, à Floriane, Julien, Xavier, Laure-Aléa, Arnold et Margot.

Aux belles rencontres de l'ICL et particulièrement à Mathilde C, Jean-Baptiste, Laurène, Mathilde M, Jean-François, Mathilde C2C, Nicolas, Simon et Natacha.

Aux barbus de Verdun avec qui toute cette aventure a commencé.

A mes co-internes picards. 
A mes très chers parents. Je vous remercie de tout mon cœur pour votre patience, votre amour et vos encouragements quotidiens.

A Eloi, mon « petit » frère. J'ai confiance en toi.

A mes très chers grands parents, Bonpapa et Bonne-Maman Leleu, Bomamie et Bonpapa Carette. Un immense Merci pour votre amour et votre bienveillance à notre égard.

A Tonton et Nounou, pour m'avoir donné le goût de l'effort. Merci pour votre amour.

A mes beaux-parents. Je vous remercie pour votre accueil dans votre si belle et grande famille.

A mes beaux-frères et belles-sœurs, qui sont comme des frères et sœurs.

A mes neveux et nièces, particulièrement à mes filleuls Aldric et Diane.

A mes très chers amis et témoins, Raphaëlle, Marin, Louise et Charles.

Aux begees lillois, particulièrement à Marie et Valentin, Marion, Estelle et Augustin, Thomas, Inès, Grégoire et Camille, Charlotte, Augustin Chef, Vincent et Camille.

Aux lorrains du Nord.

A nos plus belles rencontres de Lorraine,

Jean et Mathilde, votre amitié compte beaucoup pour nous,

Père François Weber, quelle joie de t'avoir rencontré,

A nouveau, les barbus de Verdun, particulièrement à Fanny et Dorian, Perrine et David, Clotilde, Céline, Aymeric et Lucie, Manu et Madlyne, Marine et Thibault. Julie et Jérémie, Samantha, Samuel et Valentine. 
A mon mari, Hubert.

Merci pour ton amour et ton dévouement sans faille à notre famille. Je te demande pardon pour ces longues périodes où je n'ai pas su me rendre disponible et te remercie de ton mon cœur pour ta patience à toute épreuve. Cette soutenance marque la fin d'une belle aventure lorraine et laisse place à une nouvelle étape de notre vie, ensemble, enfin.

Ce travail est aussi le tien, et je profite de ces quelques lignes pour te dire comme je t'aime.

A mon fils, Joseph.

Ton arrivée dans notre vie fait notre plus grand bonheur. Merci mon petit garçon pour ta gentillesse et ton beau sourire sur ton si doux visage. Nous t'aimons tellement. 


\section{SERMENT}

«Au moment d'être admise à exercer la médecine, je promets et je jure d'être fidèle aux lois de l'honneur et de la probité. Mon premier souci sera de rétablir, de préserver ou de promouvoir la santé dans tous ses éléments, physiques et mentaux, individuels et sociaux. Je respecterai toutes les personnes, leur autonomie et leur volonté, sans aucune discrimination selon leur état ou leurs convictions. J'interviendrai pour les protéger si elles sont affaiblies, vulnérables ou menacées dans leur intégrité ou leur dignité. Même sous la contrainte, je ne ferai pas usage de mes connaissances contre les lois de l'humanité. J'informerai les patients des décisions envisagées, de leurs raisons et de leurs conséquences. Je ne tromperai jamais leur confiance et n'exploiterai pas le pouvoir hérité des circonstances pour forcer les consciences. Je donnerai mes soins à l'indigent et à quiconque me les demandera. Je ne me laisserai pas influencer par la soif du gain ou la recherche de la gloire.

Admise dans l'intimité des personnes, je tairai les secrets qui me sont confiés. Reçue à l'intérieur des maisons, je respecterai les secrets des foyers et ma conduite ne servira pas à corrompre les mœurs. Je ferai tout pour soulager les souffrances. Je ne prolongerai pas abusivement les agonies. Je ne provoquerai jamais la mort délibérément.

Je préserverai l'indépendance nécessaire à l'accomplissement de ma mission. Je n'entreprendrai rien qui dépasse mes compétences. Je les entretiendrai et les perfectionnerai pour assurer au mieux les services qui me seront demandés.

J'apporterai mon aide à mes confrères ainsi qu'à leurs familles dans l'adversité.

Que les hommes et mes confrères m'accordent leur estime si je suis fidèle à mes promesses ; que je sois déshonorée et méprisée si j'y manque ». 


\section{TABLE DES MATIERES}

ABRÉVIATIONS 20

I. INTRODUCTION

I. 1 BRONCHOPNEUMOPATHIE CHRONIQUE OBSTRUCTIVE 22

I. 1.1 DEFINITION DE LA BRONCHOPNEUMOPATHIE CHRONIQUE OBSTRUCTIVE (BPCO) 22

I. 1.2 FACTEURS DE RISQUE, COMORBIDITES ET FACTEURS PRONOSTIQUES DE LA BPCO .23

I. 1.3 PREVALENCE DE LA BPCO .25

I. 1.4 PHYSIOPATHOLOGIE DE LA BPCO .26

I. 1.5 PRISE EN CHARGE THERAPEUTIQUE DE LA BPCO 27

I. 2 DYSPNEE 31

I. 2.1 DEFINITION DYSPNEE 31

I. 2.2 PHYSIOPATHOLOGIE DE LA DYSPNEE 33

I. 2.3 OUTILS DISPONIBLES AFIN D'EVALUER LA DYSPNEE 34

I. 2.4 LA DYSPNEE DANS LE CADRE DE LA BPCO 36

I. 2.5 PRISE EN CHARGE THERAPEUTIQUE DE LA DYSPNEE 38

II. ETUDE 40

II. 1 INTRODUCTION 40

II. 2 METHODES 42

II. 2. 1 PREMIERE PARTIE : étude prospective descriptive de cohorte 42

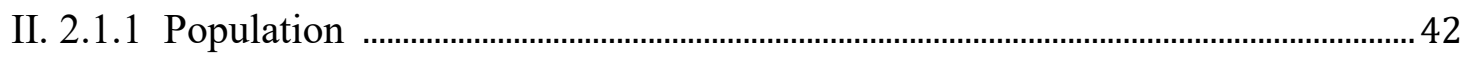

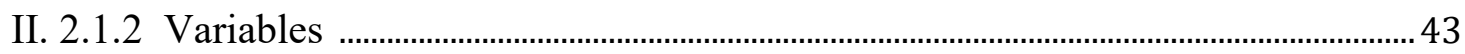

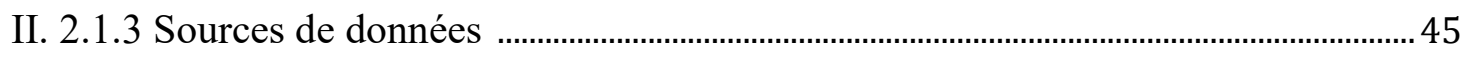


II. 2.1.4 Analyse statistique

II. 2.2 DEUXIEME PARTIE : étude déclarative multicentrique ...............................................46

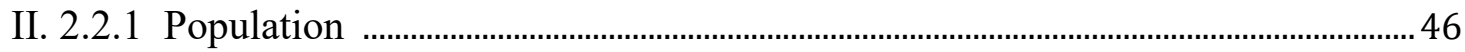

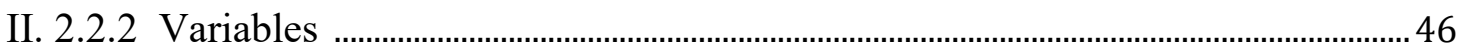

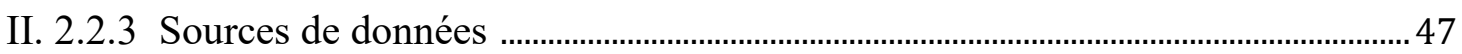

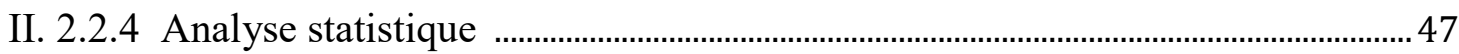

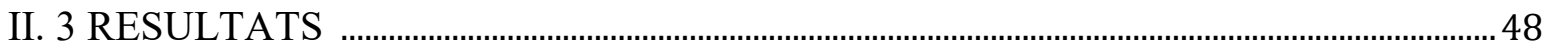

II. 3.1 RESULTATS PREMIERE PARTIE ………………………………............................... 48

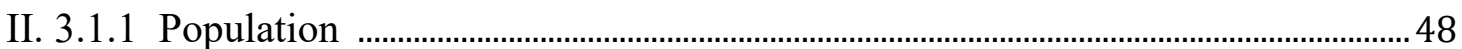

II. 3.1.2 Liens entre le stade de dyspnée (selon l'échelle mMRC) et les différents paramètres cliniques, gazométriques et spirométriques ......................................................... 49

II. 3.1.3 Etude des patients dyspnéiques sévères et dyspnéiques réfractaires ................54

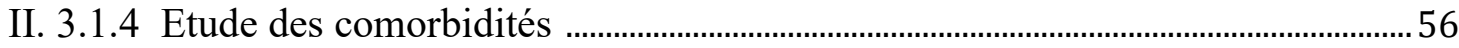

II. 3.1.5 Etude des autres symptômes ……………………………………………………....57

II. 3.1.6 Symptômes anxio-dépressifs (SAD) et prise en charge le cas échéant ............58

II. 3.1.7 Prise en charge thérapeutique de la BPCO et de la dyspnée .................................59

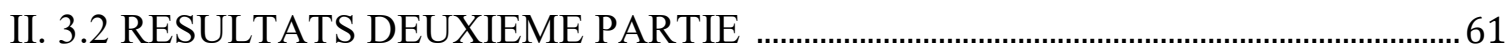

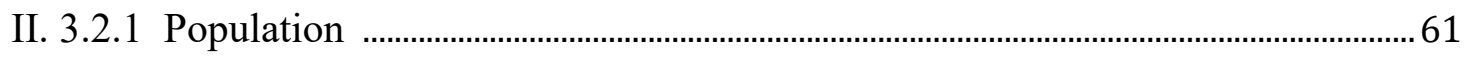

II. 3.2.2 Interventions non pharmacologiques à visée eupnéisante chez les patients atteints de BPCO : réponses au questionnaire

II. 3.2.3 Traitement pharmacologique symptomatique de la dyspnée chez les patients atteints de BPCO : réponses au questionnaire

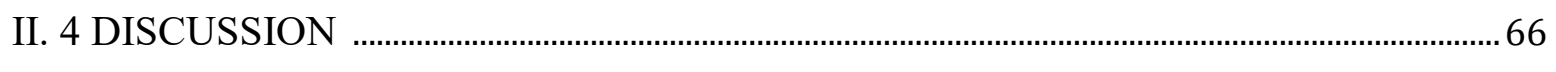

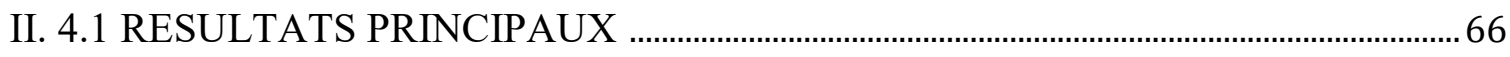

II. 4.1.1 Evaluation de la dyspnée ........................................................................................6

II. 4.1.2 Dyspnée et comorbidités .......................................................................................6 68

II. 4.1.3 BPCO, dyspnée et symptômes anxio-dépressif (SAD) ............................................69

II. 4.1.4 Recours aux interventions non pharmacologiques ..................................................... 70

II. 4.1.5 Traitement pharmacologique étiologique de la BPCO par bronchodilatateurs des patients atteints de dyspnée sévère et réfractaire ................................................................ 71

II. 4.1.6 Recours aux morphiniques à visée eupnéisante ......................................................... 71

II. 4.2 FORCES ET LIMITES …………………...................................................................... 74 


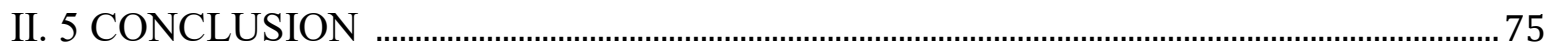

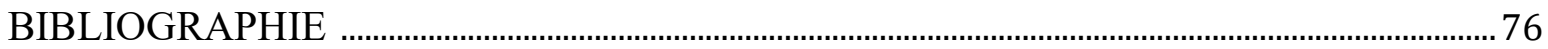

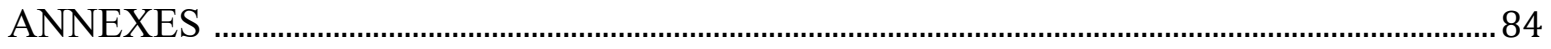




\section{ABRÉVIATIONS}

ADL : Activities of Daily Living

AMM : Autorisation de Mise sur le Marché

AVC : Accident Vasculaire Cérébral

BODE (index) : Body mass index, airflow Obstruction, Dyspnea, Exercise

BPCO : Bronchopneumopathie Chronique Obstructive

BZD : Benzodiazépines

CRF : Capacité Résiduelle Fonctionnelle

CSI : Corticostéroïdes Inhalés

CVF : Capacité Vitale Forcée

DEP : Débit Expiratoire de Pointe

EFR : Explorations Fonctionnelles Respiratoires

EVA : Echelle Visuelle Analogique

GOLD (comité) : Global Initiative for Chronic Obstructive Lung Disease

HAD : Hospital Anxiety and Depression

HTA : HyperTension Artérielle

HTP : HyperTension Pulmonaire

iBPCO : Initiatives BPCO

LABA : Long-Acting $\beta 2$ Agonist

LAMA : Long-Acting Muscarinic acetylcholine receptor Antagonist

IMC : Indice de Masse Corporelle

MDP : Multidimensionnal Dyspnea Profile

mMRC : échelle de dyspnée du Medical Research Council modifiée

NYHA : New York Heart Association 
OLD : Oxygénothérapie Longue Durée

OMS : Organisation Mondiale de la Santé

PA : Paquets-Années

$\mathrm{PaO} 2$ : Pression partielle en oxygène

PAP : Pression Artérielle Pulmonaire

PNN : Polynucléaires neutrophiles

RR : Réhabilitation Respiratoire

SABA : Short-Acting $\beta 2$ Agonist

SAD : Symptômes Anxio-Dépressifs

SAHOS : Syndrome d'Apnées Hypopnées Obstructives du Sommeil

SAMA : Short-Acting Muscarinic acetylcholine receptor Antagonist

SGRQ : Saint George’s respiratory Questionnaire

SPLF : Société de Pneumologie de la Langue Française

TVO : Trouble Ventilatoire Obstructif

VEMS : Volume Expiratoire Maximal à la première Seconde

VRI : Volume de Réserve Inspiratoire

VSRQ : Visual Simplified Respiratory Questionnaire

VT : Volume Courant 


\section{INTRODUCTION}

\section{1 BRONCHOPNEUMOPATHIE CHRONIQUE OBSTRUCTIVE}

\section{1.1 DEFINITION DE LA BRONCHOPNEUMOPATHIE CHRONIQUE OBSTRUCTIVE}

(BPCO):

La BPCO est une maladie respiratoire chronique caractérisée par des symptômes respiratoires et un trouble ventilatoire obstructifs persistants, dus à des anomalies alvéolaires ou bronchiques habituellement secondaires à l'exposition à des particules nocives ou des gaz (tabagisme, expositions professionnelles et domestiques) $(1,2)$.

Le diagnostic de BPCO est suspecté en cas de symptomatologie de bronchite chronique. La définition de la bronchite chronique est clinique, avec la présence d'une toux et d'expectorations chroniques, au moins 3 mois par an et depuis au moins deux années consécutives, sans autre cause identifiée. Ces symptômes doivent faire rechercher une BPCO par spirométrie.

En effet le diagnostic de trouble ventilatoire obstructif (TVO) est fondé sur la mesure spirométrique du volume expiratoire maximal à la première seconde (VEMS) et de la capacité vitale forcée (CVF). Le TVO de la BPCO est défini par un rapport VEMS/CVF $<70 \%$ après administration d'un bronchodilatateur (1).

La dyspnée est un maître symptôme de la BPCO, elle peut être évaluée à l'aide d'échelles validées comme la modified Medical Research Council (mMRC) dyspnea scale (3). 


\section{$\underline{\mathrm{LA} \mathrm{BPCO} \text { : }}$}

Les facteurs de risques de BPCO sont en premier lieu le tabac, puis les facteurs génétiques, l'exposition professionnelle, la pollution atmosphérique et domestique, l'existence d'un asthme. La tuberculose ainsi que l'alimentation pourraient également avoir un impact sur le développement de la BPCO (4).

La BPCO a longtemps été considérée comme une maladie respiratoire isolée. Cependant les patients atteints de BPCO présentent souvent d'autres pathologies chroniques. Charlson et al. (5) rapportent que la BPCO est associée à une ou plusieurs comorbidités dans environ $65 \%$ des cas. Les patients ayant plus de deux comorbidités ne représentent que $26 \%$ de la population, mais sont à l'origine de plus de $50 \%$ des dépenses de soin. Ces comorbidités ont un impact important sur les symptômes, le pronostic vital et les coûts de traitement. Une prise en charge thérapeutique intégrant les différentes pathologies présentes chez un même patient pourrait améliorer l'histoire naturelle de la BPCO (6). Les relations entre les comorbidités cardiovasculaires et la BPCO sont complexes. Ces pathologies recouvrent des facteurs de risque communs tels que le vieillissement, le tabagisme et la composante génétique. Cependant, les travaux de Sin et al. (7) suggèrent qu'un VEMS abaissé est un facteur de risque de mortalité cardiovasculaire à part entière, indépendant de l'âge, du sexe, et du tabagisme. L'inflammation systémique de bas grade au cours de la BPCO favoriserait également la survenue de maladies cardiovasculaires (8). Le syndrome anxio-dépressif est une comorbidité fréquente de la BPCO (9). Le score Hospital Anxiety and Depression (HAD), validé dans la BPCO, permet de détecter les symptômes d'anxiété et de dépression. Il consiste en 14 items conduisant à 2 scores différents concernant la dépression et l'anxiété. Les résultats varient de 0 à 21 , un score supérieur ou égal à 11 indique un état clinique significatif de dépression ou d'anxiété (10). 
La sévérité de la BPCO peut notamment être appréciée par la sévérité de l'obstruction bronchique : la classification GOLD (Global Initiative for Chronic Obstructive Lung Disease) en 2009 établit 4 stades dont le niveau de sévérité dépend du VEMS post bronchodilatateur. L'altération du VEMS chez les patients BPCO est bien corrélée à la mortalité. La valeur prédite est définie par des abaques, prenant en compte l'âge, le sexe, la taille et l'origine ethnique.

Le VEMS n'est pas le seul marqueur de la sévérité et du pronostic de la BPCO. Les symptômes, leur intensité, notamment la dyspnée, et les comorbidités sont pris en compte dans la prise en charge de la BPCO (11).

Figure I.1. Classification GOLD de la BPCO, stade de sévérité selon la spirométrie.

\begin{tabular}{|c|c|c|}
\hline \multicolumn{3}{|c|}{ Classification de ta BPCO en stades de sévérité } \\
\hline Stade 1: léger & VEMS/CVF $<70 \%$ & VEMS $\geq 80 \%$ valeur prédite \\
\hline Stade II : modéré & & $50 \%$ s VEMS $<80 \%$ valeur prédite \\
\hline Stade III : sévère & & $30 \% \leq$ VEMS $<50 \%$ valeur prédite \\
\hline \multirow[t]{2}{*}{ Stade IV: très sévere } & & VEMS $<30 \%$ valeur prédite \\
\hline & & $\begin{array}{l}\text { ou VEMS < } 50 \% \text { valeur prédite avec } \\
\text { insuffisance respiratoire chronique grave }\end{array}$ \\
\hline
\end{tabular}

Parmi les facteurs pronostiques de la BPCO (12) on retient la fonction respiratoire avec le VEMS (13), la sévérité et la fréquence des exacerbations (14), la dyspnée (15), les comorbidités cardiovasculaires, neuro-psychiatriques et la dénutrition (16-18). Aucun facteur pronostique pris isolément ne permet de déterminer le pronostic pour un individu donné. Le score composite BODE (valeur de 0 à 10) apparaît comme le meilleur facteur pronostique de la survie (19). Il prend en compte l'indice de masse corporelle (Body mass index), le VEMS post-bronchodilatateur exprimé en pourcentage des valeurs prédites (airflow Obstruction), le score de dyspnée mesuré par l'échelle mMRC (Dyspnea) et la distance exprimée en mètres, parcourue lors d'une épreuve de marche de 6 minutes (Exercise) (19). 
Figure I.2. Index BODE.

\begin{tabular}{|c|c|c|c|c|c|}
\hline & & 0 & 1 & 2 & 3 \\
\hline B & $\operatorname{IMC}\left(\mathrm{Kg} / \mathrm{m}^{2}\right)$ & $<21$ & $\geq 21$ & & \\
\hline 0 & VEMS ( $\%$ de la valeur prédite) & 265 & $51-64$ & $36-50$ & $\leq 35$ \\
\hline D & MMRC $(0.4)$ & $0-1$ & 2 & 3 & 4 \\
\hline E & $\begin{array}{l}\text { Distance parcourue lors du test } \\
\text { de marche de } 6 \text { minutes }(\mathrm{m})\end{array}$ & $\geq 350$ & $250 \cdot 349$ & $150-249$ & $\leq 149$ \\
\hline
\end{tabular}

Concernant les exacerbations de BPCO, il n'existe pas de définition consensuelle. Celles-ci sont définies par une majoration des symptômes respiratoires au-delà des variations quotidiennes d'une durée de plus de $48 \mathrm{~h}$ et justifiant une modification de traitement. Les critères utilisés pour définir une exacerbation sont la dyspnée, la toux et les expectorations avec une majoration en purulence et en volume (20).

Les infections respiratoires virales, bactériennes, ou mixtes, représentent la cause la plus fréquemment identifiée d'exacerbation (21).

\section{1.3 PREVALENCE DE LA BPCO :}

Concernant la BPCO en France selon une étude parue en 2010 (22), on retrouve une prévalence de la BPCO de 7,5\%, une incidence qui tend à se stabiliser chez l'homme et augmenter chez la femme, 200000 adultes âgés de 25 ans ou plus pris en charge en affection de longue durée pour une insuffisance respiratoire chronique grave ou une BPCO sévère, 93 000 adultes traités par oxygénothérapie de longue durée, un taux brut de mortalité de 41/100 000 chez les hommes et 17/100 000 chez les femmes âgées de 45 ans et plus.

La BPCO est une des principales causes de morbidité et de mortalité dans le monde entier et entraîne un fardeau économique et social croissant. D'ici 2020, l'Organisation mondiale de la 
santé (OMS) estime que la BPCO constitue la cinquième cause de handicap majeur (23) et pourrait représenter, en 2030, la quatrième cause de décès dans le monde (24).

\section{1.4 PHYSIOPATHOLOGIE DE LA BPCO :}

La BPCO est la conséquence de l'inhalation chronique d'agents irritants, le tabac en premier lieu, provoquant une réponse inflammatoire anormale et chronique des voies aériennes. Cette réponse inflammatoire chronique affecte les bronches, provoque des lésions anatomiques et fonctionnelles du poumon et perturbe les mécanismes de défense. Il en résulte une atteinte des petites voies aériennes associant inflammation et remodelage bronchique responsables d'une obstruction bronchique intrinsèque. De plus, la maladie du parenchyme pulmonaire, appelée emphysème, est responsable d'une diminution du calibre bronchique par une perte des attaches alvéolaires des bronches, ainsi que d'une diminution de la pression de rétraction élastique pulmonaire. L'ensemble de ces anomalies induit une réduction importante des débits expiratoires.

Concernant l'inflammation pulmonaire on constate dans le cadre de la BPCO une augmentation des polynucléaires neutrophiles (PNN), des macrophages, des lymphocytes T ainsi qu'un déséquilibre des lymphocytes CD4+/CD8+ cytotoxiques dans tout l'arbre bronchique et le parenchyme pulmonaire. Il existe une corrélation entre le nombre de PNN, de macrophages et de lymphocytes $\mathrm{T}$ et le déclin de la fonction respiratoire $(2,25,26)$. Il existe une inflammation systémique chronique chez les patients atteints de BPCO même à l'état stable. Par rapport à des sujets sains, les patients atteints de BPCO ont des taux plus élevés de fibrinogène plasmatique, protéine $\mathrm{C}$ réactive ultrasensible, leucocytes et TNF- alpha (27). L'inflammation systémique dans le cadre de la BPCO est associée aux manifestations générales de la maladie telles que l'amaigrissement, l'ostéoporose, les dysfonctions musculaires et squelettiques ainsi que les pathologies cardiovasculaires (26). 


\section{1.5 PRISE EN CHARGE THERAPEUTIQUE DE LA BPCO :}

. Sevrage tabagique :

L'arrêt du tabagisme est la principale mesure susceptible de ralentir la progression de l'obstruction bronchique et de retarder l'apparition de l'insuffisance respiratoire. L'arrêt du tabac diminue la mortalité globale mais également la mortalité par cancer bronchopulmonaire et pathologie cardiovasculaire (28-30).

Les stratégies d'aide au sevrage sont similaires à celles utilisées en population générale, à commencer par le conseil minimal (31).

. Réhabilitation respiratoire (12,32):

La réhabilitation respiratoire $(\mathrm{RR})$ est un ensemble de soins personnalisés, dispensés au patient atteint d'une maladie respiratoire chronique, par une équipe transdisciplinaire. Elle a pour objectif de réduire les symptômes, d'optimiser les conditions physiques et psychosociales et de diminuer les coûts de santé. Est candidat à la RR tout malade atteint de BPCO présentant, malgré un traitement médicamenteux optimal, une dyspnée entraînant une diminution des activités physiques quotidiennes. Le programme de soins proposé par la RR comprend plusieurs composantes : entrainement à l'exercice, éducation thérapeutique, prise en charge psychologique, sevrage tabagique, suivi nutritionnel et prise en charge sociale. Ce programme peut être réalisé en hospitalisation, en ambulatoire ou au domicile. Une méta-analyse (33) a recensé 6 essais randomisés contrôlés regroupant 140 patients avec RR et 90 patients dans le groupe contrôle. La RR améliore la dyspnée, la capacité à l'exercice et la qualité de vie. Elle permet la diminution de la fréquence des réadmissions et de la mortalité au décours d'une hospitalisation. 
. Vaccination grippale et anti-pneumococcique (12):

La vaccination grippale annuelle est recommandée chez les patients qui présentent une BPCO. Il est recommandé de vacciner ces patients par le vaccin polyosidique pneumococcique.

. Traitement pharmacologique étiologique et symptomatique :

Les recommandations de la SPLF (12) concernant le traitement de la BPCO sont présentées dans la Figure I.3.

Figure I.3. Traitement de la BPCO en fonction du stade de sévérité spirométrique SPLF 2009.

\begin{tabular}{|c|c|c|c|}
\hline 1: Léger & II : Modéré & III : Sévère & IV: Très sévère \\
\hline \multicolumn{4}{|c|}{ VEMS $/ C V<0,7$} \\
\hline $\begin{array}{l}\text { VEMS } \geq 80 \% \\
\text { de la théorique }\end{array}$ & $\begin{array}{l}50 \% \leq \text { VEMS }<80 \% \\
\text { de la théorique }\end{array}$ & $\begin{array}{l}30 \% \leq \text { VEMS }<50 \% \\
\text { de la théorique }\end{array}$ & $\begin{array}{l}\text { VEMS < } 30 \% \\
\text { de la théorique } \\
\text { ou VEMS }<50 \% \\
\text { de la valeur théorique } \\
\text { avec insuffisance } \\
\text { respiratoire chrorique }\end{array}$ \\
\hline \multicolumn{4}{|c|}{\begin{tabular}{l|l} 
& $\begin{array}{l}\text { Un ou plusieurs bronchodilatateurs de longue durée d'action } \\
\text { Réhabilitation }\end{array}$
\end{tabular}} \\
\hline & & $\begin{array}{l}\text { Glucocorticostéroì } \\
\text { d'association fixes } \\
\text { (veMs } \times \operatorname{cox} \text { pour solmet' }\end{array}$ & $\begin{array}{l}\text { s inhatés sous forme } \\
\text { exacerbations répétées } \\
\text { muticasone) }\end{array}$ \\
\hline 5 & $a^{\prime \prime}$ & & $\begin{array}{l}\text { Oxygénothérapie } \\
\text { longue durée } \\
\text { si insuffisance } \\
\text { respiratoire chronique } \\
\text { Traitements } \\
\text { chirurgicaux }\end{array}$ \\
\hline
\end{tabular}

Sous le terme de bronchodilatateurs sont regroupés deux classes de médicaments. D’une part, les bêta-2 agonistes, de courte (salbutamol, terbutaline) ou longue durée d'action (salméterol, formoterol) et d'autre part les anticholinergiques de courte (ipratropium) ou longue durée 
d'action (tiotropium). Ces bronchodilatateurs augmentent le VEMS ou améliorent d'autres paramètres spirométriques, en diminuant le tonus musculaire lisse. Il n'existe pas de relation entre l'augmentation des débits expiratoires (VEMS, DEP) et l'amélioration de la symptomatologie. Cette dernière est plus vraisemblablement liée à une réduction de la distension thoracique, corrélée à la réduction de la dyspnée au repos et à l'amélioration de la tolérance à l'exercice. Ainsi, le bénéfice clinique rapporté par le malade prime sur l'éventuelle amélioration des débits expiratoires.

L'efficacité du traitement par bronchodilatateurs inhalés, associés ou non à des cortico des inhalés, peut être observée sur la dyspnée et le débit de pointe dans les 15 jours qui suivent le début du traitement (12).

Concernant la dyspnée dans le cadre de la BPCO, il faut souligner qu'une seule étude avait ce critère pour objectif principal et a démontré un bénéfice statistiquement significatif en faveur de l'association indacatérol/glycopyrronium, cliniquement pertinent versus placebo mais non versus tiotropium (34).

La SPLF $(35,36)$ a récemment proposé une alternative aux recommandations internationales 2011-2016 du comité GOLD concernant la prise en charge de la BPCO. En effet, les symptômes tels que la dyspnée seraient davantage pris en compte et guideraient la prise en charge pharmacologique de la BPCO. 
Figure I.4. Proposition d'algorithme de prise en charge thérapeutique dans la BPCO (36).

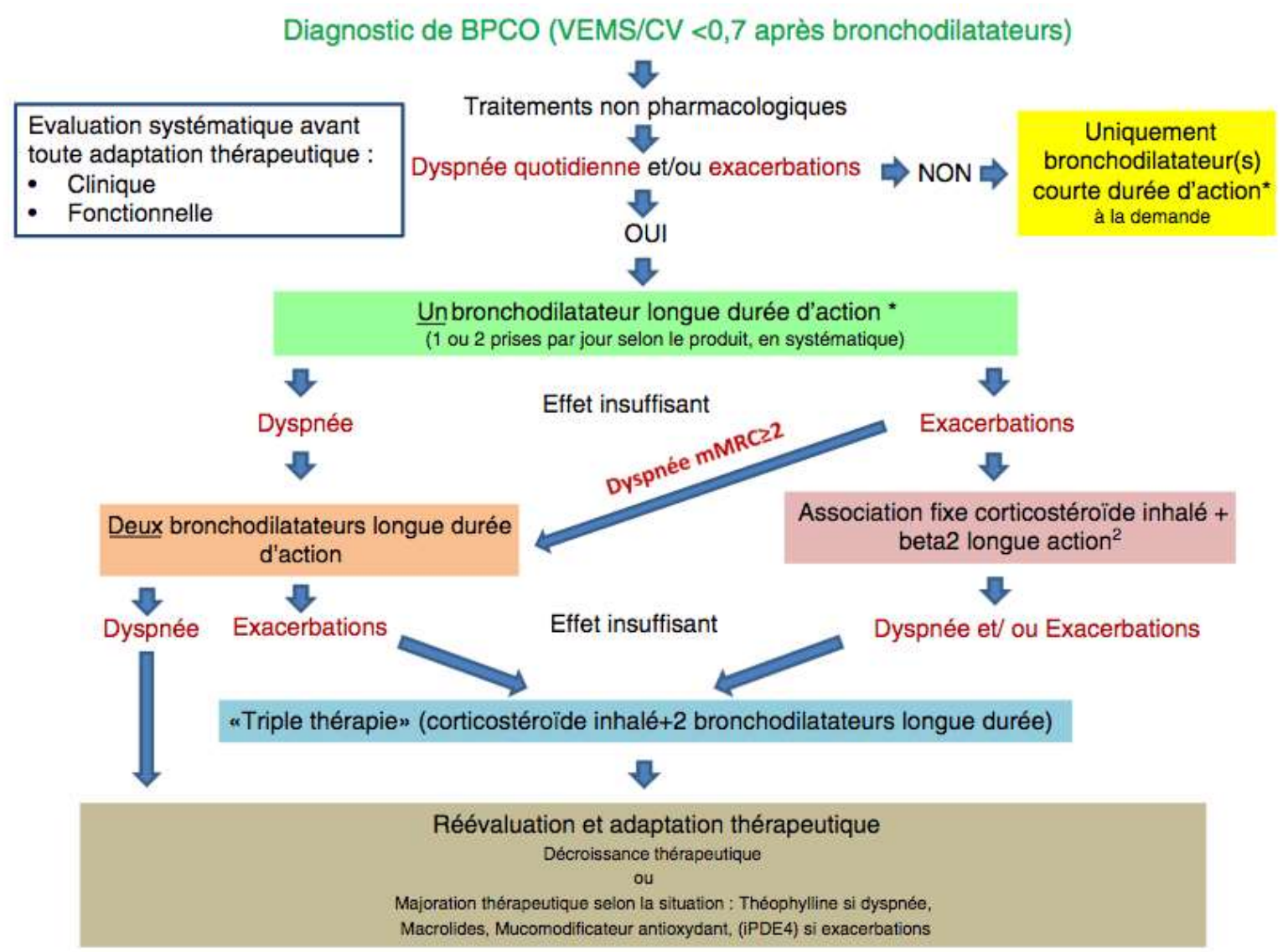

. Oxygénothérapie $(37,38)$ :

Les indications de l'oxygénothérapie longue durée (OLD) sont strictement codifiées chez les patients atteints de BPCO au stade d'insuffisance respiratoire. La prescription d'OLD est justifiée lorsque deux mesures des gaz du sang au repos, en état stable, sous traitement médical optimal et à au moins quinze jours d'intervalle montrent une $\mathrm{PaO} 2 \leq 55 \mathrm{mmHg}$. Lorsque la $\mathrm{PaO} 2$ est entre 56 et $59 \mathrm{mmHg}$, l'OLD est indiquée s'il existe l'un ou l'autre des éléments suivants : une hypertension pulmonaire (PAP moyenne $>25 \mathrm{mmHg}$ ), des signes cliniques de cœur pulmonaire chronique, une désaturation artérielle en oxygène pendant le sommeil sans relation avec un syndrome d'apnées du sommeil ( $\mathrm{SpO} 2<90 \%$ plus de $30 \%$ du temps d'enregistrement), une polyglobulie (Hématocrite $>55 \%$ ). 


\section{2 DYSPNEE}

\section{2.1 DEFINITION DYSPNEE (39):}

L'actuelle définition consensuelle de la dyspnée est la suivante : « La dyspnée est une expérience subjective d'inconfort respiratoire faite de sensations qualitativement distinctes et d'intensité variable », " Le terme dyspnée décrit un ensemble de sensations respiratoires, survenant à l'exercice ou au repos, dont l'affect est désagréable, et qui peuvent s'accompagner de modifications ventilatoires et neurovégétatives. L'évaluation de son intensité est subjective. Elle entraîne des réponses physiologiques et comportementales susceptibles d'altérer le mode et la qualité de vie des patients. » (40). On retrouve quatre notions fondamentales dans cette définition. Concernant la subjectivité : le discours du patient suffit à établir la présence d'une dyspnée. La deuxième est celle de « multimodalité » : il existe différentes sensations dyspnéiques (soif d'air, suffocation, effort respiratoire excessif, oppression thoracique...) comme il existe différentes sensations douloureuses ; elles procèdent généralement de mécanismes physiopathologiques distincts $(41)$.

La troisième notion est celle de « multidimensionnalité » : on ne parle de dyspnée que si la dimension sensorielle, appelée percept, est associée à un affect négatif, l'intensité de ces dimensions peut varier indépendamment (exemple de l'analogie musicale : le volume sonore peut varier indépendamment du plaisir d'écoute, il n'est pas nécessaire que ce volume soit fort pour qu'une musique que l'on n'apprécie pas soit ressentie comme désagréable). En l'absence d'affect négatif, on ne parle pas de dyspnée mais de «sensations respiratoires » 
La dernière notion fondamentale est celle de "diffusivité », en effet la dyspnée a un impact non seulement respiratoire, mais également neurovégétatif, émotionnel et comportemental (42).

La dyspnée est une source de souffrance, tant physique, du fait de son retentissement (limitation des capacités d'exercice), que psychologique (anxiété, dépression, désinvestissement), qui altère la qualité de vie et qui nécessite d'être soulagée indépendamment de sa cause, à titre de symptôme.

Figure I.5. Modèle psychophysiologique de la dyspnée (39).

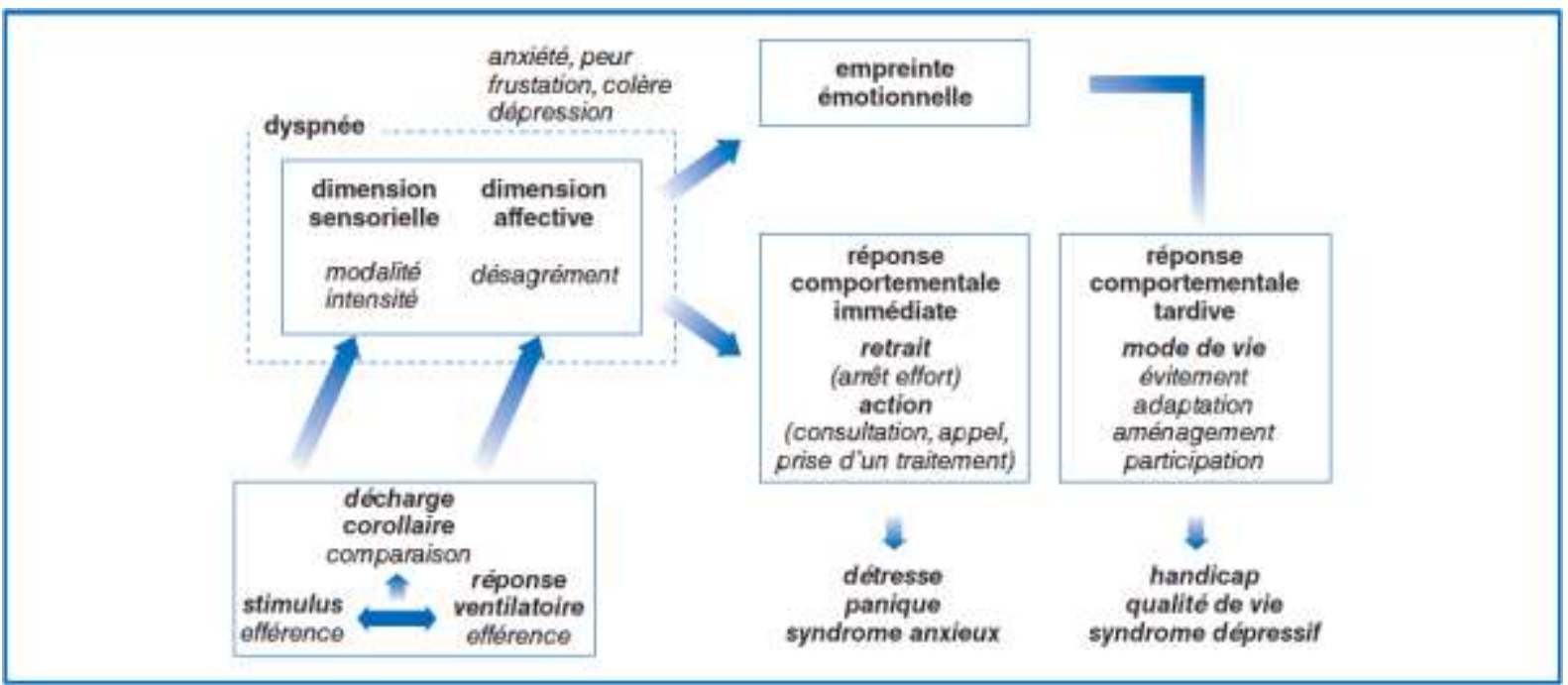

La dyspnée est extrêmement fréquente, y compris lorsque l'on considère des populations hors circuit respiratoire (43). Sa fréquence augmente avec l'âge.

La dyspnée réfractaire se caractérise par une dyspnée persistante malgré un traitement optimal de la pathologie sous-jacente, pour une durée de plus de 3 mois, avec une intensité importante (survenant au repos, ou au moindre effort) (44).

La dyspnée résiduelle se caractérise par une dyspnée persistante malgré un traitement optimal de la pathologie sous-jacente, depuis plus de 3 mois donc chronique, qui se révèle invalidante mais sans notion d'intensité comme pour la dyspnée réfractaire. 


\section{2.2 PHYSIOPATHOLOGIE DE LA DYSPNEE (39):}

Il semble que les voies afférentes impliquées dans la dyspnée soient multiples et différentes selon le stimulus en cause (45).

Les afférences mises en cause dans la dyspnée véhiculent les signaux provenant de récepteurs intra-pulmonaires et bronchiques médiés par le nerf vague (45), de mécanorécepteurs des voies aériennes supérieures et de la paroi thoracique ainsi que de chémorécepteurs centraux et périphériques.

Les informations, transmises au cortex par les afférences respiratoires, sont ensuite interprétées sur le plan affectivo-cognitif. On distingue plusieurs zones d'intégration centrale de la dyspnée : l'insula antérieure droite, le vermis cérébelleux, le cortex cingulaire et l'amygdale $(46,47)$.

Parallèlement à ces événements neurosensoriels, la dyspnée implique des réponses comportementales immédiates et à long terme, influencées par sa prise en charge (48). 
Figure I.6. Modèle physiopathologique de la dyspnée (39).

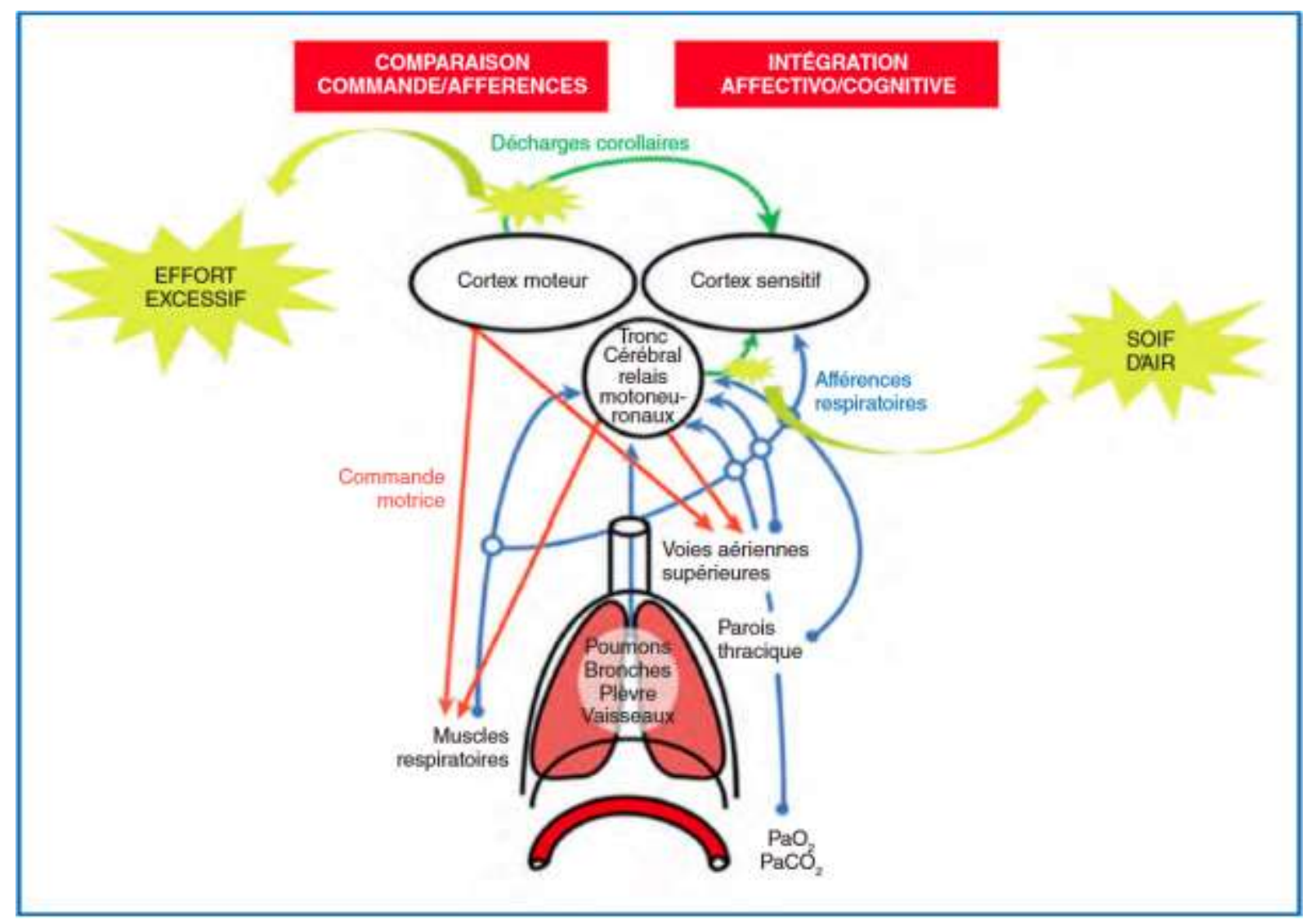

\section{2.3 OUTILS DISPONIBLES AFIN D'EVALUER LA DYSPNEE (40)}

L'échelle visuelle analogique (EVA) est l'échelle de référence qui mesure l'inconfort respiratoire, 0 correspondant à l'absence totale d'inconfort et 10 à une sensation insupportable. L'échelle de Borg guide le patient au moyen d'une catégorie sémantique. D'autres échelles évaluent le retentissement de la dyspnée sur les activités de la vie quotidienne des patients ainsi que sur leur qualité de vie au cours de certaines pathologies spécifiques. C'est le cas de la modified Medical Research Council (mMRC) dyspnea scale dans le cadre de la BPCO (3) et de la New York Heart Association (NYHA) pour les patients souffrant d'insuffisance cardiaque. 
Figure I.7. Echelle de dyspnée mMRC.

\section{Echelle de dyspnée modified Medical Research Council (mMRC) dyspnea scale :}

Stade 0 : Dyspnée pour des efforts intenses ;

Stade 1 : Dyspnée lors de la marche rapide ou en pente ;

Stade 2 : Dyspnée à la marche sur terrain plat en suivant quelqu'un de son âge ;

Stade 3 : Dyspnée obligeant à s'arrêter pour reprendre son souffle après quelques minutes ou une centaine de mètres sur terrain plat ;

Stade 4 : Dyspnée au moindre effort.

Ces échelles et scores ne prennent en compte qu'une seule dimension de la dyspnée. Ces dernières années, de nouveaux questionnaires comme le Multidimensionnal Dyspnea Profile (MDP) et le Dyspnea 12 ont été développés dans l'objectif de prendre en compte l'aspect multidimensionnel de la dyspnée.

Le score Dyspnea 12 a été élaboré à partir de patients atteints d'insuffisance respiratoire chronique, les auteurs ont retenu douze items, les premiers représentent la composante sensorielle de la dyspnée et les derniers représentent la composante affective, gradée de 0 (absent) à 3 (sévère). Ce score, notamment validé dans la BPCO, est bien corrélé au stade mMRC et à l'échelle de Borg (49). Le Multidimensionnal Dyspnea Profile (MDP) est constitué d'échelles graduées de 0 à 10 qui quantifient les différentes composantes de la dyspnée. Il distingue ainsi la composante affective immédiate soit l'inconfort respiratoire, la composante sensorielle c'est à dire la nature de la perception («effort excessif»,, «soif d'air», «constriction thoracique», «effort mental») et enfin la réponse émotionnelle secondaire (anxiété, peur) (48). 


\section{2.4 LA DYSPNEE DANS LE CADRE DE LA BPCO :}

La dyspnée est le symptôme le plus fréquemment rapporté par les patients souffrant de BPCO de stade avancé (50). De nombreux patients atteints de BPCO sévère subissent une réduction de leur qualité de vie secondaire à la dyspnée (51) . D'après une enquête téléphonique en Europe et en Amérique du Nord en 2000 (52,53), 20\% des patients ressentent une dyspnée de repos, $24 \%$ souffrent d'une dyspnée à la parole, soit un quart d'entre eux présenteraient une dyspnée réfractaire dans l'hypothèse d'un traitement optimal. Trente pour cent des patients se plaignent d'une dyspnée pour des activités quotidiennes, 70\% rapportent une dyspnée pour un effort peu important tel que la montée de quelques marches.

Cette dyspnée existe quel que soit le stade GOLD. Seuls $18 \%$ des patients ne rapportent aucune dyspnée (mMRC 0), la dyspnée réfractaire est fréquente dans la BPCO, touchant jusqu'à 30\% des patients, y compris lorsque l'obstruction bronchique est peu importante (53). Cependant, la fréquence de cette dyspnée réfractaire augmente à mesure que le stade GOLD augmente.

L'obstruction bronchique qui caractérise la BPCO est à l'origine d'une limitation de débit expiratoire. En conséquence, lorsque le temps expiratoire diminue, la quantité d'air inspiré précédemment ne peut être totalement évacuée. À chaque cycle respiratoire, davantage d'air s'accumule dans le poumon et une distension pulmonaire apparaît progressivement. Cette distension se constitue plus ou moins progressivement selon la fréquence respiratoire et l'importance de la limitation de débit expiratoire. Il existe deux types de distension : statique (augmente le volume de relaxation du fait de la réduction des forces de rétraction élastique du poumon), et dynamique (écarte la CRF du volume de relaxation) (54). Ce faisant, elle crédite l'acte respiratoire de deux contraintes successives qui rendent l'inspiration difficile voire intolérable pour les patients les plus sévères. En effet, l'augmentation du volume pulmonaire 
liée à la limitation des débits expiratoires et à l'inflation dynamique impose une augmentation de travail inspiratoire que les muscles respiratoires ne peuvent satisfaire dans ce contexte de BPCO. C'est ce déséquilibre entre l'effecteur que sont les muscles et la commande qui provoque la dyspnée d'effort ou travail respiratoire excessif.

Les traitements médicamenteux de la BPCO visent essentiellement à diminuer l'obstruction bronchique et ainsi sa conséquence fonctionnelle, la distension pulmonaire (55). Les bronchodilatateurs, en particulier les $\beta 2$ mimétiques, réduisent la distension dynamique et augmentent la capacité inspiratoire. De ce fait on constate, sous traitement bronchodilatateur versus placebo, une augmentation du volume courant (VT), associé à une diminution de la vitesse de réduction de la capacité inspiratoire. Ceci a pour effet une diminution de la sensation de dyspnée à temps d'exercice égal (le point d'inflexion reste toujours à 0,51 pour le volume de réserve inspiratoire (VRI), mais est atteint plus tardivement sous bronchodilatateur). La sensation de dyspnée est directement liée aux modifications du VT, la dyspnée est donc inversement corrélée à l'augmentation du VT sous bronchodilatateurs, à l'augmentation de la capacité inspiratoire dynamique et à l'augmentation de la capacité inspiratoire de repos (56).

Par ailleurs, l'hypoxémie et l'hypercapnie induites par la modification des rapports ventilation-perfusion dans la BPCO entraînent toutes deux une augmentation de l'intensité de la commande respiratoire centrale. C'est l'inadéquation entre l'augmentation de cette commande respiratoire et la réponse ventilatoire insuffisante du fait de l'effecteur défectueux qui induit la dyspnée de type « soif d'air » $(57,58)$.

Les comorbidités de la BPCO peuvent elles aussi participer à l'aggravation de la dyspnée (6). En effet les pathologies cardiovasculaires, l'obésité et les symptômes anxio-dépressifs sont pourvoyeurs de dyspnée. 


\section{2.5 PRISE EN CHARGE THERAPEUTIQUE DE LA DYSPNEE:}

Le caractère multidimensionnel de la dyspnée impose une prise en charge pluridisciplinaire, associant un traitement étiologique et symptomatique ainsi qu'une prise en charge psychologique.

. Prise en charge étiologique de la dyspnée dans le cadre de la BPCO, approche non pharmacologique et pharmacologique :

Les traitements spécifiques types RR et bronchodilatateurs ont été décrits ci-dessus dans le chapitre consacré à la BPCO.

Le traitement de l'obstruction bronchique et de la distension pulmonaire par des bronchodilatateurs spécifiques sont déterminants pour la prise en charge de la dyspnée, mais est insuffisant voire inopérant à long terme sur les incapacités si aucune prise en charge spécifique n'accompagne cette prescription (52). Les traitements recommandés de la dyspnée dans le cadre de la BPCO sont la RR, la prise en charge des comorbidités et un traitement bronchodilatateur optimal.

. Prise en charge symptomatique de la dyspnée, approche non pharmacologique et pharmacologiques :

- Morphiniques et anxiolytiques :

Les opioïdes systémiques constituent actuellement le seul traitement symptomatique de la dyspnée, hors AMM, dont l'efficacité a été démontrée sur un petit nombre de patients sélectionnés $(59,60)$.

Les morphiniques agissent à différents niveaux. L'action des opio des sur la commande ventilatoire centrale est bien connue mais c'est une action peu importante et ne suffit pas à elle seule à expliquer l'effet bénéfique des opio des dans ce contexte. Les opio des agissent 
donc, comme pour la douleur, sur les voies de transmission centrale de la dyspnée, mais également sur le système limbique dont l'insula et l'amygdale porteuses de récepteurs mu opioïdes. $(59,61)$

Les anxiolytiques ne sont pas reconnus comme traitement de la dyspnée. Chez certains patients ayant une composante affective importante de la dyspnée, les anxiolytiques peuvent être efficaces. Toutefois l'utilisation de benzodiazépines (BZD) et d'opio des à fortes doses dans le cadre d'une insuffisance respiratoire chronique est associée à une augmentation de la mortalité, particulièrement en cas d'utilisation concomitante (62).

- Les autres voies thérapeutiques :

Via une action sur les afférences respiratoires, de nouvelles voies thérapeutiques peuvent être envisagée.

L'hypnose médicale est une voie thérapeutique intéressante. Elle permet de réduire les composantes affectives et sensorielles de la dyspnée en agissant sur le cortex limbique et particulièrement sur le cortex cingulaire (63).

La stimulation des récepteurs à l'étirement via le furosémide (diurétique de l'anse) en nébulisation mime une inflation pulmonaire et trompe le cerveau sur le volume pulmonaire généré par le système respiratoire. Néanmoins, son bénéfice demeure hétérogène et non prédictible $(64,65)$. La stimulation des récepteurs des voies aériennes supérieures améliore la sensation de dyspnée, ce qui peut expliquer l'efficacité de l'air sec ou de l'oxygène médical à un débit suffisant chez les patients non hypoxémiques souffrant de dyspnée réfractaire (66). La stimulation du nerf trijumeau par un jet d'air froid sur le visage réalise un gaiting et ainsi bloque les afférences respiratoires médian la sensation de dyspnée (67). L’impact de la stimulation olfactive par le L-menthol sur la dyspnée a récemment fait l'objet d'une étude (68). 


\section{ETUDE}

\section{1 INTRODUCTION}

\section{Contexte}

La dyspnée est une des principales doléances des patients atteints de BPCO. Elle est définie de façon consensuelle comme « une expérience subjective d'inconfort respiratoire faite de sensations qualitativement distinctes et d'intensité variable ». La dyspnée réfractaire se caractérise par une dyspnée persistant malgré un traitement optimal de la pathologie sousjacente, durant plus de 3 mois, avec une intensité importante. La dyspnée est source de souffrance, tant physique du fait de son retentissement, que psychologique, elle altère la qualité de vie et nécessite d'être soulagée indépendamment de sa cause, à titre de symptôme (40).

La bronchopneumopathie chronique obstructive (BPCO) est une maladie respiratoire chronique définie par une obstruction non complètement réversible et permanente des voies aériennes, secondaire à une exposition toxique (tabagisme, expositions professionnelles et domestiques). La dyspnée est le symptôme le plus fréquemment rapporté par les patients atteints de BPCO de stade avancé (50). Elle est responsable de handicap et représente un facteur pronostique de la maladie (12). L'évaluation et la prise en charge étiologique et symptomatique de la dyspnée font partie intégrante de la prise en charge globale du patient atteint de BPCO.

\section{Objectifs}

L'objectif principal de cette étude est la description du symptôme de dyspnée et de sa prise en charge chez les patients atteints de BPCO en Lorraine. 
Les objectifs secondaires sont les suivants :

Etudier les caractéristiques de la dyspnée, en fonction de sa sévérité, chez les patients atteints de BPCO à l'état stable.

Déterminer l'impact des comorbidités sur la dyspnée dans le cadre de la BPCO.

Déterminer le lien entre dyspnée et symptômes anxio-dépressifs (SAD) dans le cadre de la BPCO et leur prise en charge le cas échéant. 


\section{2 METHODES}

Notre étude est réalisée en deux parties :

\section{2. 1 PREMIERE PARTIE : étude prospective descriptive de cohorte}

La première partie est une étude prospective descriptive de cohorte. INITIATIVES BPCO (iBPCO) est une cohorte de patients atteints de BPCO, diagnostiqués par spirométrie (rapport VEMS/CVF post-bronchodilatateur $<70 \%$ ), à l'état stable, au sein de 17 départements de pneumologie d'hôpitaux universitaires situés dans toute la France, dont le CHRU de Nancy. Seules les caractéristiques démographiques et la spirométrie sont obligatoires pour inclure un patient. L'étude a été approuvée par le Comité d'éthique de Versailles en France (numéro: 04479), et tous les sujets ont fourni un consentement éclairé écrit.

\section{2.1.1 Population :}

La population étudiée concerne les 123 premiers patients atteints de BPCO, tous stades confondus, à l'état stable, inclus dans la cohorte iBPCO (annexe 1), du 01/06/2015 au 25/02/2016 au sein du département de pneumologie du CHRU de Nancy.

Les critères d'inclusion sont ceux de la cohorte iBPCO et concernent les patients atteints de BPCO, tous stades confondus (examen clinique et spirométrie confirmant le diagnostic), à l'état stable.

Les critères de non inclusion sont ceux de la cohorte iBPCO, soit un état clinique non stable (exacerbation). Il n’y a pas de restriction concernant les comorbidités. 


\section{2.1.2 Variables :}

Les variables recueillies étaient les suivantes :

- liées aux patients : l'âge, le sexe, l'Indice de Masse Corporelle (IMC), l'intoxication tabagique et le nombre de paquets-années (PA), les comorbidités respiratoires, métaboliques, cardiovasculaires, une hypertension pulmonaire (HTP) et la notion de douleurs chroniques.

Les symptômes anxio-dépressifs sont évalués par le score Hospital Anxiety and Depression (HAD).

- $\quad$ liées aux scores de qualité de vie : Activities of Daily Living (ADL), Visual Simplified Respiratory Questionnaire (VSRQ ) et Saint George's respiratory Questionnaire (SGRQ).

- liées à la BPCO : la dyspnée selon l'échelle mMRC, le nombre d'exacerbations l'année précédant l'inclusion, la présence de symptômes quotidiens comme la toux et les expectorations, le stade GOLD, les résultats spirométriques (CVF, VEMS) et gazométriques $(\mathrm{pH}, \mathrm{PaO} 2, \mathrm{PaCO} 2)$ de moins de quatre mois.

- liées aux traitements non pharmacologiques et pharmacologiques de la BPCO : réhabilitation respiratoire (RR), bronchodilatateurs inhalés (LAMA, LABA, SAMA, SABA), corticostéroïdes inhalés (CSI), et l'oxygénothérapie (O2) en cas d'insuffisance respiratoire chronique.

- liées aux autres traitements : antalgiques de palier 2 ou 3, anxiolytiques type benzodiazépines (BZD), antidépresseurs, traitements à visée cardiovasculaire. 
Dans notre étude, les variables suivantes sont définies comme suit :

- L'obésité est définie par un IMC supérieur à $30 \mathrm{~kg} \cdot \mathrm{m}^{-2}$ et la maigreur par un IMC inférieur à $18 \mathrm{~kg} \cdot \mathrm{m}^{-2}$

- L'HTP est définie par une pression artérielle pulmonaire moyenne supérieure à $25 \mathrm{mmHg}$ au cathétérisme cardiaque droit.

- Le score HAD est utilisé pour détecter les symptômes anxieux et dépressifs des patients. Ce questionnaire, validé dans la BPCO, consiste en 14 items conduisant à 2 scores différents concernant la dépression et l'anxiété. Les résultats vont de 0 à 21 , un score supérieur ou égal à 11 indique un état clinique significatif de dépression ou d'anxiété (10).

- La dyspnée sévère est définie par un stade mMRC supérieur ou égal à 3 , qui correspond à un retentissement clinique majeur de la dyspnée dans la vie quotidienne.

Parmi les patients dyspnéiques sévères (mMRC 3 et 4), une dyspnée réfractaire est retenue si la dyspnée sévère persiste malgré un traitement optimal de la pathologie sous-jacente, durant plus de 3 mois, avec une intensité importante (c'est-à-dire mMRC 3 stable, mMRC 4 stable, mMRC 3 augmentée à 4).

- Le phénotype « exacerbateur fréquent » est défini par un nombre d'exacerbation de BPCO supérieur ou égal à 2 par an.

- La prise en charge non pharmacologique de la dyspnée dans le cadre de la BPCO est d'une part l'activité physique et d'autre part la réhabilitation respiratoire (RR).

- La prise en charge pharmacologique étiologique des patients atteints de BPCO qui ont une dyspnée sévère et réfractaire était considérée comme optimale si elle est conforme aux recommandations GOLD 2015 (2) et de la SPLF 2009 (12), c'est à dire le recours à un doublet de bronchodilatateurs (association LABA-LAMA) et ajout d'un CSI au doublet de bronchodilatateurs en cas de phénotype exacerbateur fréquent. 


\section{2.1.3 Sources de données :}

Le recueil des données a été réalisé via la cohorte iBPCO, complétés par les données du dossier médical accessible au CHU de Nancy (recueil exhaustif des comorbidités et des traitements retrouvés dans les courriers médicaux).

\section{2.1.4 Analyse statistique :}

Les variables qualitatives sont décrites en effectifs et pourcentages. La normalité de la distribution des paramètres quantitatifs a été étudiée par le test du Shapiro-Wilk. En fonction de la normalité de la distribution, ces paramètres ont été décrits par la moyenne et l'écart-type ou la médiane, l'étendue ou l'intervalle inter-quartile.

Les comparaisons entre deux groupes ont été réalisées avec des tests non paramétriques, test de Chi 2 ou Fischer pour les données qualitatives. Les coefficients de corrélation utilisés sont les coefficients de Spearman ou de Pearson, selon l'effectif.

Une valeur de $\mathrm{p} \leq 0,05$ est considérée comme statistiquement significative. L'analyse des résultats a été effectuée à l'aide du logiciel GraphpadPrism 6.00 (GraphpadPrismSoftware Inc., San Diego,CA) ou SAS ${ }^{8} 9.2$ statistical software. 


\section{2.2 DEUXIEME PARTIE : étude déclarative multicentrique}

La deuxième partie de l'étude est une évaluation des pratiques professionnelles concernant la prise en charge de la dyspnée chez les patients atteints de BPCO. Il s'agit d'une étude déclarative multicentrique via un questionnaire réalisé par le groupe dyspnée de la SPLF sous la direction du Dr Morélot-Panzini et adapté aux patients BPCO. Ce questionnaire a été envoyé aux Docteurs en médecine de Lorraine, spécialistes en pneumologie, quel que soit leur type d'exercice (CHU, CHR, établissement privé, libéral, activité mixte).

\section{2.2.1 Population :}

La population étudiée concerne les pneumologues de Lorraine.

\section{2.2.2 Variables :}

Description du questionnaire : (annexe 2)

- Un premier volet de 6 items concernant les données démographiques (caractéristiques personnelles et professionnelles des médecins répondants), dont un item permettant d'identifier les répondants, afin d'éviter les réponses en doublon et de relancer sélectivement les non - répondants. Les données du questionnaire étaient ensuite exploitées de manière anonyme.

- Un deuxième volet de 10 items, dont 8 concernaient la prise en charge symptomatique non pharmacologique et pharmacologique de la dyspnée des patients atteints de BPCO dans différents contextes (ambulatoire, hospitalier, fin de vie), 2 items interrogeaient les médecins quant à leur réticence à prescrire des morphiniques ou des benzodiazépines à visée eupnéisante dans le cadre de la BPCO. 
La notion de «médecins inclueurs » concerne les pneumologues qui ont participé à l'étude iBPCO en incluant leurs patients dans cette cohorte. Les «médecins non-inclueurs» représentent les autres pneumologues.

\section{2.2.3 Sources de données :}

Le questionnaire a été adressé par courriel via Google Forms ${ }^{\circledR}$ aux pneumologues de Lorraine à l'aide de l'annuaire du Collège Lorrain de Pathologie Thoracique (CLPT), du 27 février au 27 mars 2017.

\section{2.2.4 Analyse statistique :}

L'analyse des réponses au questionnaire est exclusivement descriptive. L'effectif des différents groupes étudiés ne nous permet pas d'avoir recours à des tests statistiques. Les variables qualitatives sont décrites en effectifs et pourcentages. 


\section{3 RESULTATS}

\section{3.1 RESULTATS PREMIERE PARTIE :}

\section{3.1.1 Population}

Le diagramme de flux des patients inclus dans l'étude est présenté en Figure II.1.

Entre le 01/06/2015 et le 25/02/2016, 123 patients étaient éligibles à la cohorte iBPCO,

3 patients ont refusé de remplir le questionnaire, soit un total de 120 patients inclus.

Figure II.1. Diagramme de flux

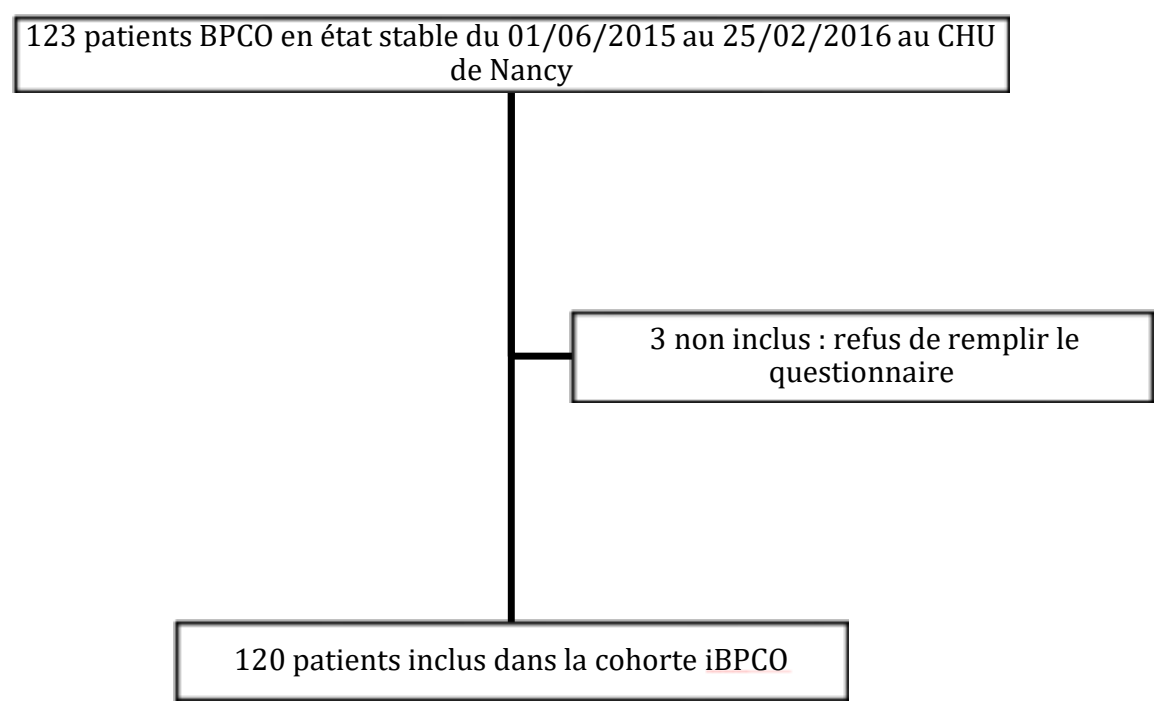

Les caractéristiques des patients inclus dans la cohorte iBPCO sont présentées en Tableau 1. 
Tableau 1. Caractéristiques des 120 patients inclus dans la cohorte iBPCO

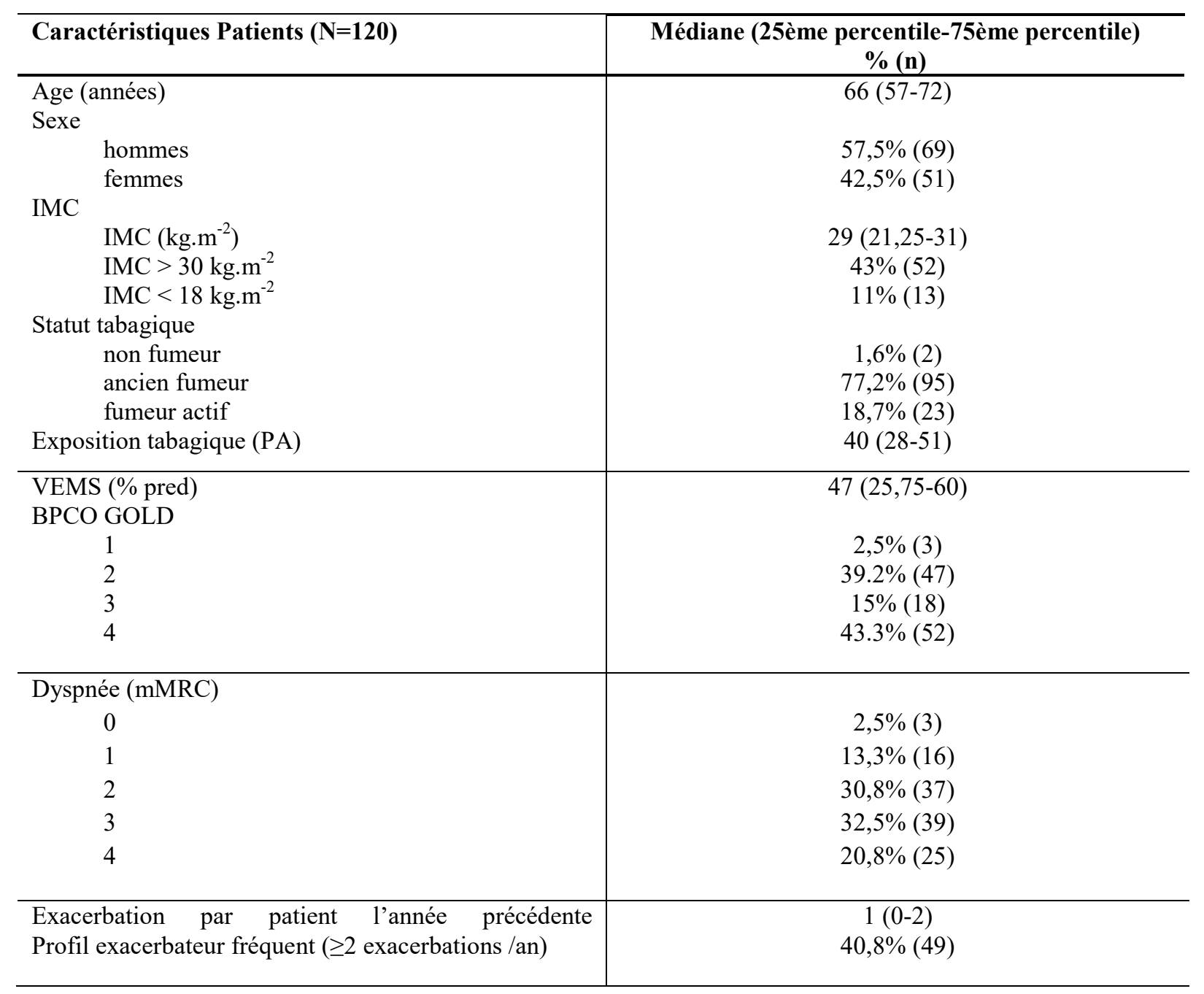

\section{3.1.2 Liens entre le stade de dyspnée (selon l'échelle mMRC) et les différents}

paramètres cliniques, gazométriques et spirométriques.

- Stade de dyspnée en fonction des paramètres cliniques :

Il n'existe pas de corrélation entre l'IMC et le stade mMRC $(r=0,1, p=0,24)$. On ne retrouve pas non plus de corrélation entre le statut tabagique et le stade de dyspnée évalué par l'échelle $\operatorname{mMRC}(\mathrm{r}=-0,06, \mathrm{p}=0,49)$. 
Le score ADL et le score VSRQ médian pour les 120 patients est respectivement de 22 (11$32)$ et $43(29-60)$.

Il existe une corrélation entre le stade de dyspnée et le questionnaire de qualité de vie ADL $(\mathrm{r}=0,64 \mathrm{p}<0,0001)$, et une corrélation négative entre la dyspnée évaluée par l'échelle mMRC et le score VSRQ ( $\mathrm{r}=-0,34 \mathrm{p}=0,0002)$ ) (Figure II.2).

Figure II.2. Dyspnée et échelles de qualité de vie (ADL et VSRQ)
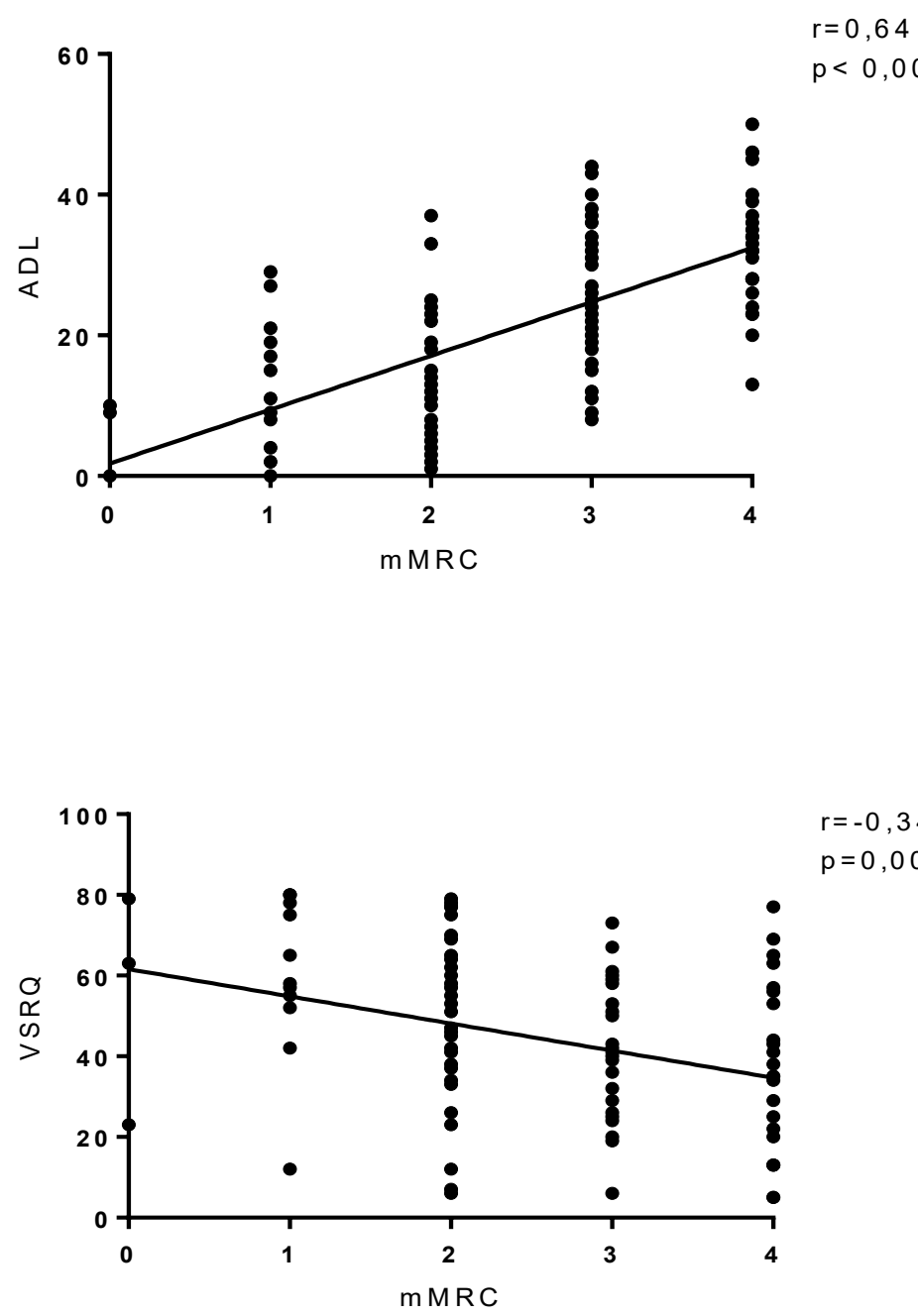

$r=-0,34$

$p=0,0002$

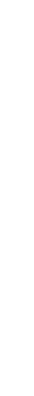

Le score SGRQ activité médian pour les 120 patients inclus est de 73 (54-93). Il existe une forte corrélation positive entre le stade de dyspnée évalué par l'échelle mMRC et 
le SGRQ activité $(r=0,69, \mathrm{p}<0,0001)$ (Figure II.3).

Figure II.3. Dyspnée et SGRQ Activité

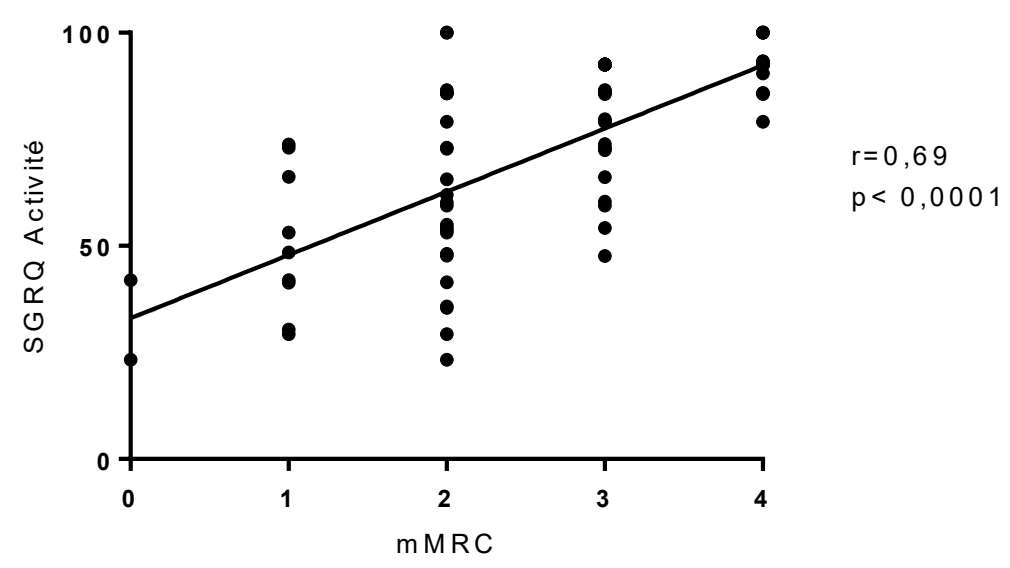

Concernant le lien entre la dyspnée et le score HAD, on retrouve une corrélation entre le stade de dyspnée évalué par l'échelle mMRC et les symptômes d'anxiété et de dépression détectés par le score HAD anxiété $(r=0,27 \mathrm{p}=0,0023)$ et le score HAD dépression $(r=0,5 \mathrm{p}<0,0001)$ (Figure II.4). 
Figure II.4. Dyspnée et score HAD anxiété et dépression
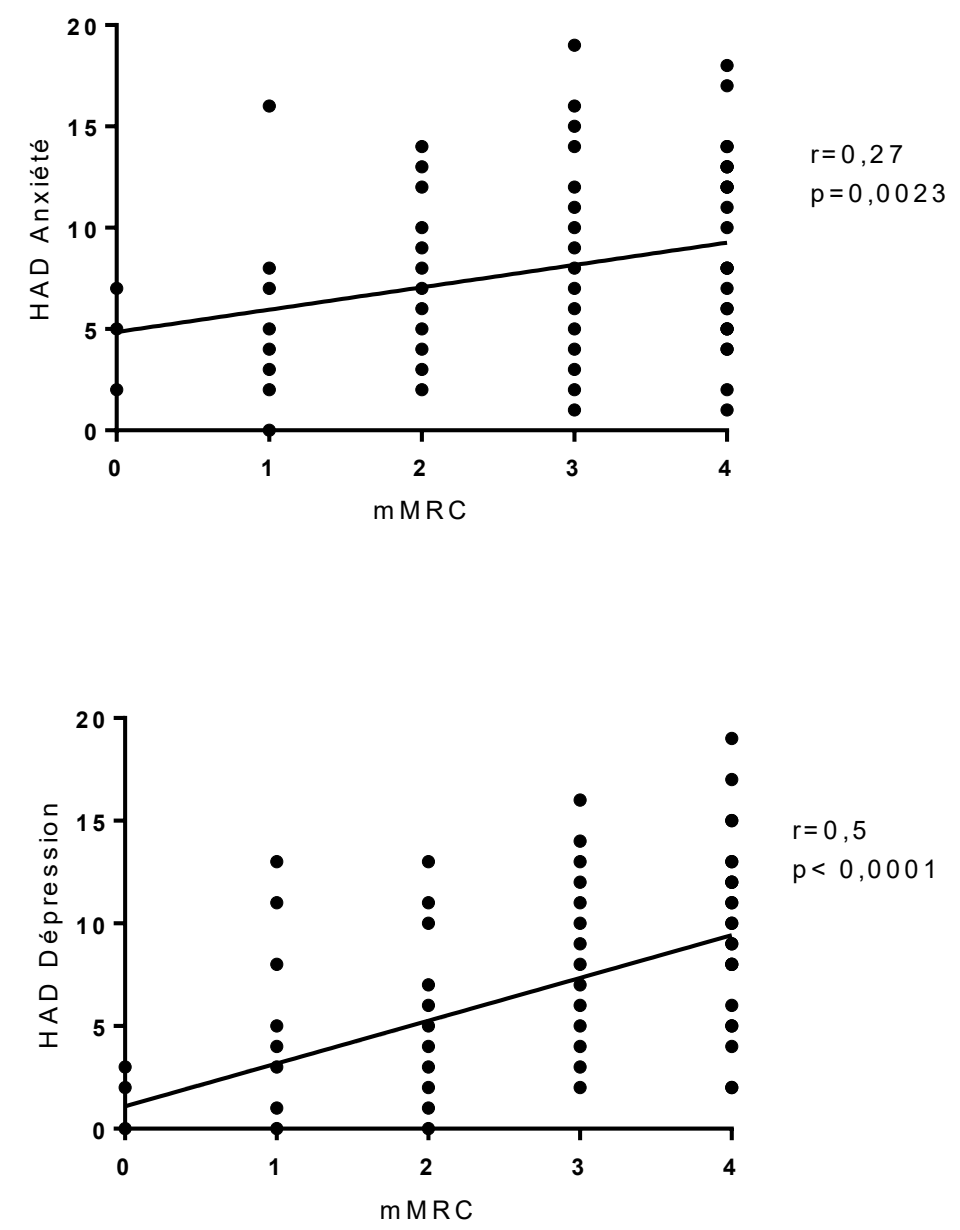

- Stade de dyspnée en fonction des échanges gazeux :

Concernant la gazométrie artérielle, on ne retrouve pas de corrélation entre la $\mathrm{PaO}_{2}$ et le stade de dyspnée, en revanche la capnie $\left(\mathrm{PaCO}_{2}\right)$ est faiblement et positivement corrélée au stade $\operatorname{mMRC}(r=0,24 \mathrm{p}=0,01)$ (Figure II.5). 
Figure II.5. Dyspnée en fonction de la capnie (mmHg).

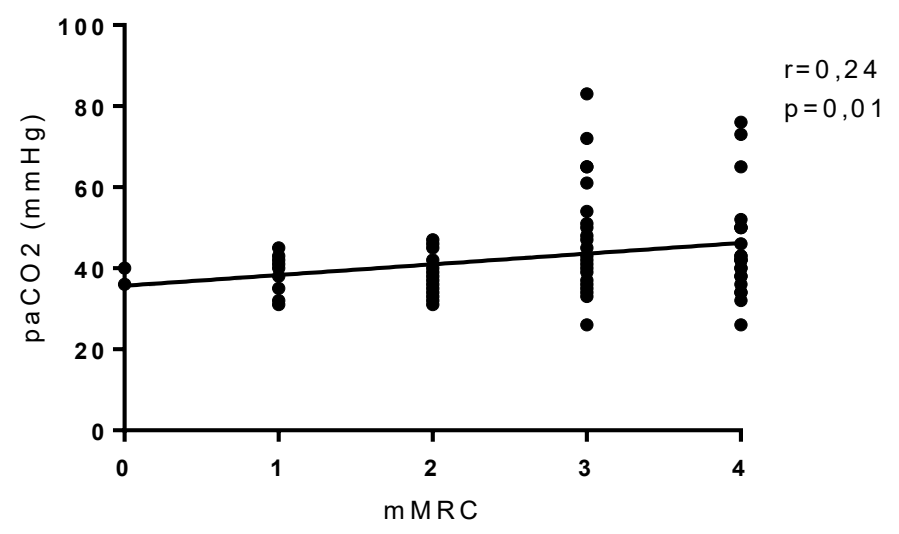

- Stade de dyspnée en fonction des épreuves fonctionnelles respiratoires (EFR) :

Il existe une corrélation entre le VEMS en pourcentage de la valeur prédite et le stade de dyspnée mMRC ( $\mathrm{r}=0,49 \mathrm{p}<0,0001)$ (Figure II.6).

Figure II.6. Dyspnée en fonction du VEMS (\%).

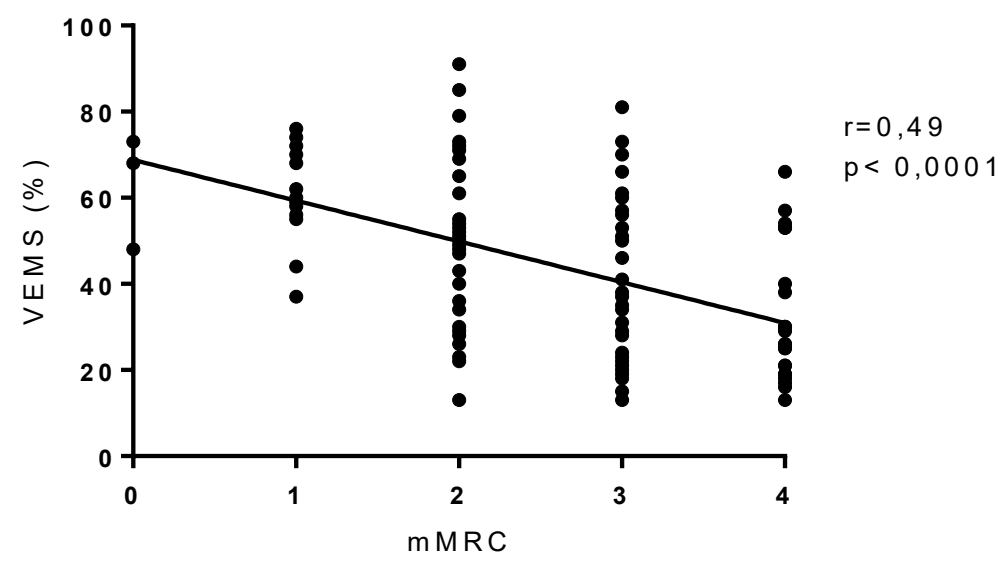




\section{3.1.3 Etude des patients dyspnéiques sévères et dyspnéiques réfractaires}

Parmi les 120 patients, 56 patients $(47 \%)$ ont une dyspnée faible à modérée $(\mathrm{mMRC}<3)$ et 64 patients $(53 \%)$ ont une dyspnée sévère $(\mathrm{mMRC}>3)$. Parmi ceux-ci 41 patients $(64 \%)$, soit 34\% de la population totale, présentent une dyspnée réfractaire (Figure II.7).

Figure II.7. Répartition des stades de dyspnée au sein des 120 patients de la cohorte iBPCO

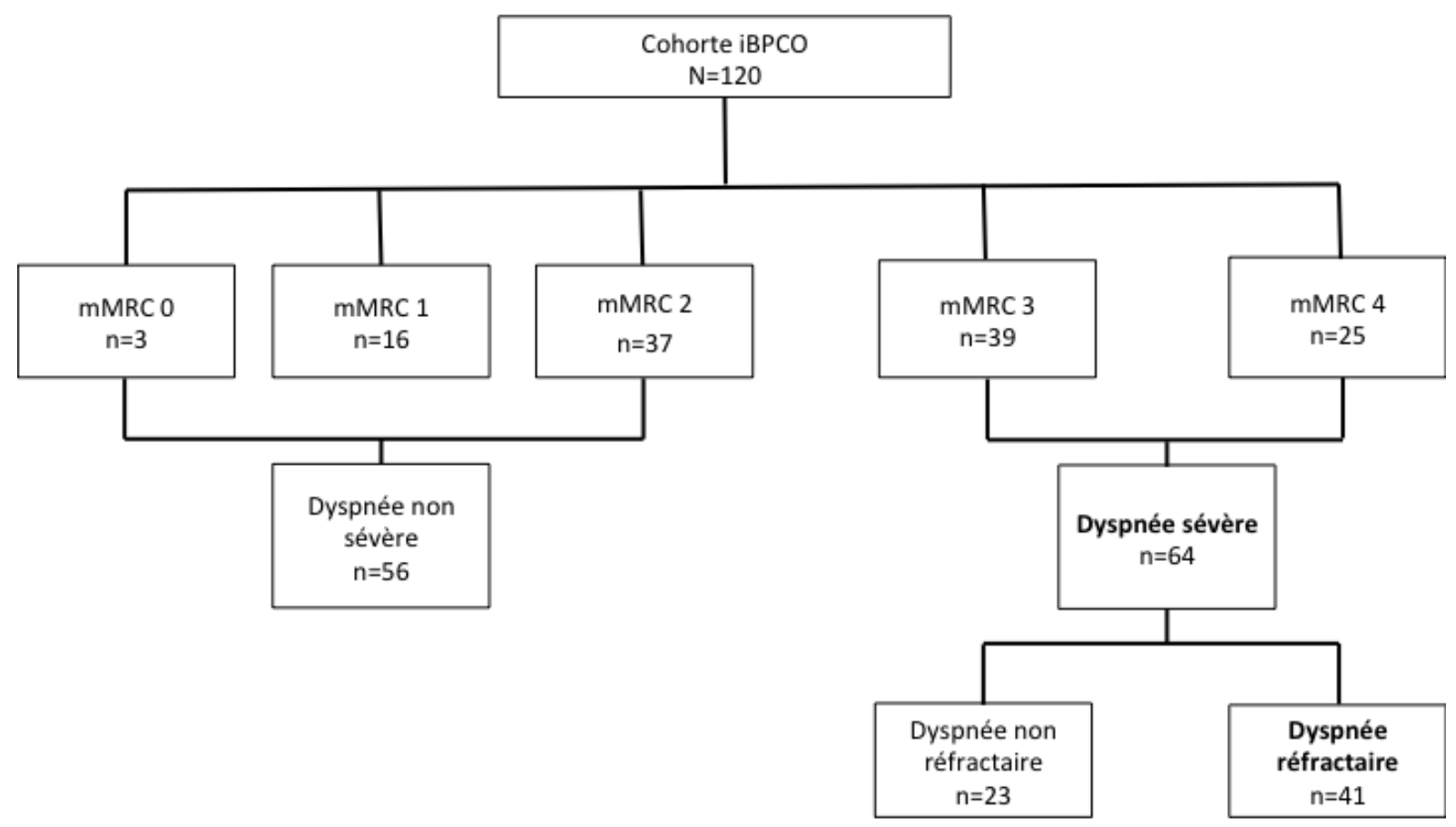

Les caractéristiques des patients ayant une dyspnée sévère sont présentées en Tableau 2. 
Tableau 2. Caractéristiques des 64 patients ayant une dyspnée sévère MRC 3-4

\begin{tabular}{|c|c|}
\hline Caractéristiques des patients dyspnéiques sévères & $\begin{array}{c}\text { Dyspnée sévère } \\
\text { mMRC } 3+4 \\
n=64\end{array}$ \\
\hline Dyspnée réfractaire & $64 \%(41)$ \\
\hline Age & $64(56-70)$ \\
\hline \multicolumn{2}{|l|}{ Sexe } \\
\hline Hommes & $62,5 \%(40)$ \\
\hline Femmes & $37,5 \%(26)$ \\
\hline \multicolumn{2}{|l|}{ BPCO GOLD } \\
\hline 1 & $1,5 \%(1)$ \\
\hline 2 & $25 \%(16)$ \\
\hline 3 & $8 \%(5)$ \\
\hline 4 & $65,5 \%(42)$ \\
\hline Exacerbateurs fréquents & $46,8 \%(30)$ \\
\hline \multicolumn{2}{|l|}{ Tabac } \\
\hline Non fumeurs & $3 \%(2)$ \\
\hline Actifs & $23,5 \%(15)$ \\
\hline Sevrés & $73,5 \%(47)$ \\
\hline \multicolumn{2}{|l|}{ IMC } \\
\hline Obèses IMC $>30$ kg.m ${ }^{2}$ & $42 \%(27)$ \\
\hline Maigres IMC $<18$ kg.m ${ }^{2}$ & $12,5 \%(8)$ \\
\hline
\end{tabular}

Les caractéristiques des patients ayant une dyspnée réfractaire sont présentées en Tableau 3.

Tableau 3. Caractéristiques des 41 patients ayant une dyspnée réfractaire

\begin{tabular}{l|c}
\hline Caractéristiques des patients dyspnéiques réfractaires & Dyspnée réfractaire \\
\hline Age & $64(56-68)$ \\
Sexe & \\
Hommes & $63,4 \%(26)$ \\
Femmes & $36,6 \%(15)$ \\
BPCO GOLD & $2,4 \%(1)$ \\
1 & $12,2 \%(5)$ \\
2 & $4,9 \%(2)$ \\
3 & $80,5 \%(33)$ \\
4 & $49 \%(20)$ \\
Exacerbateurs fréquents & $5 \%(2)$ \\
Tabac & $22 \%(9)$ \\
Non fumeurs & $73 \%(30)$ \\
Actifs & $34 \%(14)$ \\
Sevrés & $17 \%(7)$ \\
Poids & \\
Obèses IMC $>30 \mathrm{~kg} . \mathrm{m}^{2}$ & \\
Maigres IMC $<18 \mathrm{~kg} \cdot \mathrm{m}^{2}$ & \\
\hline
\end{tabular}




\section{3.1.4 Etude des comorbidités}

Le relevé des comorbidités des 120 patients, et en fonction du stade de dyspnée, est présenté en Tableau 4.

Tableau 4. Comorbidités et stade de dyspnée

\begin{tabular}{|c|c|c|c|}
\hline Comorbidités CV & $\begin{array}{l}\text { Population totale } \\
\qquad \mathrm{N}=120 \\
\end{array}$ & $\begin{array}{c}\text { Dyspnée sévère } \\
\text { mMRC 3-4 } \\
\text { n=64 } \\
\end{array}$ & $\begin{array}{r}\text { Dyspnée réfractaire } \\
n=41 \\
\end{array}$ \\
\hline $\begin{array}{l}\text { HTA } \\
\text { Coronaropathie } \\
\text { AVC } \\
\text { Troubles du rythme } \\
\text { Insuffisance cardiaque }\end{array}$ & $\begin{array}{c}50 \%(60) \\
14 \%(17) \\
5 \%(6) \\
20 \%(25) \\
16 \%(19)\end{array}$ & $\begin{array}{c}51,5 \%(33) \\
12,5 \%(8) \\
5 \%(3) \\
23 \%(15) \\
20 \%(13)\end{array}$ & $\begin{array}{c}49 \%(20) \\
7 \%(3) \\
7 \%(3) \\
15 \%(6) \\
17 \%(7)\end{array}$ \\
\hline Comorbidités autres & $\begin{array}{l}\text { Population totale } \\
\qquad \mathrm{N}=\mathbf{1 2 0}\end{array}$ & $\begin{array}{c}\text { Dyspnée sévère } \\
\text { mMRC 3-4 } \\
\text { n=64 }\end{array}$ & $\begin{array}{c}\text { Dyspnée réfractaire } \\
n=41\end{array}$ \\
\hline $\begin{array}{l}\text { Obésité } \\
\text { Diabète type } 2 \\
\text { HTP } \\
\text { SAHOS } \\
\text { Embolie pulmonaire }\end{array}$ & $\begin{array}{l}43 \%(52) \\
17 \%(21) \\
10 \%(12) \\
28 \%(34) \\
3 \%(4)\end{array}$ & $\begin{array}{c}42 \%(27) \\
19 \%(12) \\
14 \%(9) \\
31 \%(20) \\
5 \%(3)\end{array}$ & $\begin{array}{c}34 \%(14) \\
17 \%(7) \\
17 \%(7) \\
29 \%(12) \\
2 \%(1)\end{array}$ \\
\hline
\end{tabular}

$\mathrm{CV}=$ cardiovasculaires

L'étude des comorbidités chez les patients dyspnéiques sévères versus non sévères est détaillée Tableau 5. En analyse univariée, il y a significativement plus d'ostéoporose chez les patients ayant une dyspnée sévère $(\mathrm{p}=0.006)$. On ne retrouve pas de différence significative entre les deux groupes concernant les autres comorbidités, notamment concernant l'obésité, les pathologies cardiovasculaires ou l'hypertension pulmonaire. 
Tableau 5. Etude des comorbidités dyspnée sévère versus non sévère, analyse univariée.

\begin{tabular}{l|c|c|c}
\hline Comorbidités & $\begin{array}{c}\text { Dyspnée non sévère mMRC 0-1-2 } \\
\mathbf{n = 5 6}\end{array}$ & $\begin{array}{c}\text { Dyspnée sévère mMRC 3-4 } \\
\mathbf{n = 6 4}\end{array}$ & $\mathbf{p}$ \\
\hline \hline HTA & $48.2 \%(27)$ & $51.6 \%(33)$ & 0.714 \\
Coronaropathie & $16.1 \%(9)$ & $12.5 \%(8)$ & 0.576 \\
Troubles du rythme & $17.9 \%(10)$ & $23.4 \%(15)$ & 0.453 \\
Diabète type 2 & $16.1 \%(9)$ & $18.8 \%(12)$ & 0.700 \\
Insuffisance cardiaque & $10.7 \%(6)$ & $20.3 \%(13)$ & 0.151 \\
\hline \hline Obésité & $44.6 \%(25)$ & $42.2 \%(27)$ & 0.787 \\
HTP & $5.4 \%(3)$ & $14.1 \%(9)$ & 0.113 \\
SAHOS & $25.0 \%(14)$ & $31.3 \%(20)$ & 0.448 \\
Embolie pulmonaire & $1.8 \%(1)$ & $4.7 \%(3)$ & 0.622 \\
AVC & $5.4 \%(3)$ & $4.7 \%(3)$ & - \\
Cancer pulmonaire & $3.6 \%(2)$ & $3.1 \%(2)$ & - \\
Ostéoporose & $3.6 \%(2)$ & $20.3 \%(13)$ & 0.006 \\
Douleurs chroniques & $16.1 \%(9)$ & $14.1 \%(9)$ & 0.758 \\
\hline
\end{tabular}

Pour ces même paramètres, en analyse univariée, on ne retrouve pas de différence significative entre les patients dyspnéiques réfractaires et les non réfractaires, hormis les troubles du rythme $(p=0,026)$.

\section{3.1.5 Etude des autres symptômes}

On ne retrouve pas de différence significative entre les groupes dyspnée sévère versus non sévère et dyspnée réfractaire versus non réfractaire concernant les symptômes tels que la toux et les expectorations.

On ne retrouve pas non plus de différence significative entre ces groupes concernant la fréquence des exacerbations de BPCO. 


\section{3.1.6 Symptômes anxio-dépressifs (SAD) et prise en charge le cas échéant}

\section{- Prévalence des SAD :}

La prévalence des SAD chez les 120 patients de la cohorte iBPCO, et en fonction de la sévérité de la dyspnée est représentée Tableau 6.

Tableau 6. Symptômes anxio-dépressifs des 120 patients, et en fonction du stade de dyspnée

\begin{tabular}{l|c|c|c}
\hline Score HAD & $\begin{array}{c}\text { Population totale } \\
\mathbf{N}=\mathbf{1 2 0}\end{array}$ & $\begin{array}{c}\text { Dyspnée sévère mMRC 3-4 } \\
\mathbf{n = 6 4}\end{array}$ & $\begin{array}{c}\text { Dyspnée réfractaire } \\
\mathbf{n = 4 1}\end{array}$ \\
\hline HAD Anxiété $>\mathbf{1 0}$ & $23,3 \%(28)$ & $34,4 \%(22)$ & $36,6 \%(15)$ \\
HAD Dépression $>\mathbf{1 0}$ & $19,2 \%(23)$ & $29,7 \%(19)$ & $36,6 \%(15)$ \\
\hline
\end{tabular}

Les patients qui présentent une dyspnée sévère ont significativement plus de SAD évocateurs de syndrome anxio-dépressif (score HAD anxiété et/ou dépression $>10$ ) que les patients dyspnéiques non sévères (Tableau 7).

Tableau 7. Etude des symptômes anxio-dépressifs des patients dyspnéiques sévères versus non sévères.

\begin{tabular}{l|c|c|c}
\hline Score HAD & $\begin{array}{c}\text { Dyspnée non sévère mMRC 0-1-2 } \\
\mathbf{n}=\mathbf{5 6}\end{array}$ & $\begin{array}{c}\text { Dyspnée sévère mMRC 3-4 } \\
\mathbf{n}=\mathbf{6 4}\end{array}$ & $\mathbf{p}$ \\
\hline HAD anxiété $>\mathbf{1 0}$ & $88.9 \%(48) / 11.1 \%(6)$ & $65.6 \%(42) / 34.4 \%(22)$ & 0.003 \\
HAD dépression $>\mathbf{1 0}$ & $92.6 \%(50) / 7.4 \%(4)$ & $68.9 \%(42) / 31.1 \%(19)$ & 0.001 \\
\hline
\end{tabular}

En revanche on ne retrouve pas de différence significative concernant les SAD entre les patients dyspnéiques réfractaires et non réfractaires. 


\section{- $\quad$ Prise en charge des SAD :}

La prise en charge des $\mathrm{SAD}$ en fonction du tableau clinique dominé par l'anxiété ou la dépression pour les 120 patients, et en fonction de la gravité de la dyspnée est décrite Tableau 8.

Tableau 8. Prise en charge des symptômes anxio-dépressifs en fonction du score HAD anxiété, dépression, et en fonction de la gravité de la dyspnée

\begin{tabular}{l|c|c|c}
\hline HAD Anxiété $>\mathbf{1 0}$ & $\begin{array}{c}\text { Population } \\
\text { totale } \\
\mathbf{n = 2 8}\end{array}$ & $\begin{array}{c}\text { Dyspnée sévère mMRC 3-4 } \\
\mathbf{n = 2 2}\end{array}$ & $\begin{array}{c}\text { Dyspnée réfractaire } \\
\mathbf{n = 1 5}\end{array}$ \\
\hline Traitement anxiolytique & $25 \%(7)$ & $27,3 \%(6)$ & $20 \%(3)$ \\
\hline \hline HAD Dépression $>\mathbf{1 0}$ & $\begin{array}{c}\text { Population } \\
\mathbf{t o t a l e} \\
\mathbf{n}=\mathbf{2 3}\end{array}$ & $\begin{array}{c}\text { Dyspnée sévère } \mathbf{m M R C} \mathbf{3 - 4} \\
\mathbf{n}=\mathbf{1 9}\end{array}$ & $\begin{array}{c}\text { Dyspnée réfractaire } \\
\mathbf{n}=\mathbf{1 5}\end{array}$ \\
\hline Traitement antidépresseur & $13 \%(3)$ & $15,8 \%(3)$ & $13,3 \%(2)$ \\
\hline
\end{tabular}

\section{3.1.7 Prise en charge thérapeutique de la BPCO et de la dyspnée}

\section{- Recours à la RR :}

Parmi les 120 patients, 41 patients (34\%) ont bénéficié de RR.

Parmi les 64 patients dyspnéiques sévères, 26 patients (40,6\%) ont bénéficié de RR.

Parmi les 41 patients dyspnéiques réfractaires, 17 patients $(41,5 \%)$ ont bénéficié de RR.

- Traitement pharmacologique étiologique de la BPCO des patients atteints de dyspnée sévère et réfractaire :

Parmi les 64 patients qui ont une dyspnée sévère, 60 patients (94\%) bénéficient d'un traitement étiologique pharmacologique optimal en accord avec les recommandations de la 
SPLF 2009. Parmi les 30 patients qui ont une dyspnée sévère et un profil exacerbateur fréquent, 26 patients (87\%) bénéficient d'un traitement étiologique pharmacologique optimal. Parmi les 41 patients qui présentent une dyspnée réfractaire, tous ont, par définition, un traitement étiologique pharmacologique optimal. Parmi les 20 patients qui ont une dyspnée réfractaire et un phénotype exacerbateur fréquent, 1 seul patient ne bénéficie pas d'un traitement étiologique pharmacologique optimal.

\section{- Traitements des comorbidités :}

Les principales comorbidités de la BPCO sont les pathologies cardiovasculaires, l'obésité et le syndrome anxio-dépressif. Concernant la prise en charge de ces comorbidités, tous les patients qui ont des antécédents cardiovasculaires ont au moins un traitement à visée cardiovasculaire. L'obésité concerne $43 \%$ des 120 patients de notre cohorte, $42 \%$ des patients qui ont une dyspnée sévère et $34 \%$ des patients ayant une dyspnée réfractaire. Le traitement de l'obésité consiste en une démarche de perte de pondérale, nous ne disposons pas de données permettant d'évaluer cette prise en charge.

Les patients inclus sont en état stable. Aucun patient ne présente d'exacerbation de BPCO ni de tableau d'insuffisance cardiaque aiguë.

\section{- Recours aux morphiniques à visée eupnéisante :}

Aucun patient atteint de dyspnée sévère ou réfractaire ne bénéficie d'un traitement pharmacologique symptomatique par morphiniques à visée eupnéisante. Deux patients ont un traitement par morphine orale, initiée dans un contexte de douleurs chroniques (dyspnée de stade mMRC 2 dans les deux cas). 


\section{3.2 RESULTATS DEUXIEME PARTIE :}

\section{3.2.1 Population}

Entre le 27/02/2017 et le 27/03/2017, 46 médecins sur 88 médecins interrogés (soit 52\%) ont répondu au questionnaire.

Les caractéristiques des médecins répondeurs sont représentées Tableau 1a.

Tableau 1a. Caractéristiques des médecins répondeurs

\begin{tabular}{l|c}
\hline Caractéristiques médecins répondeurs (N=46) & \% (n) \\
\hline Age & $11 \%(5)$ \\
$\quad<30$ ans & $26 \%(12)$ \\
$30-39$ ans & $20 \%(9)$ \\
$40-49$ ans & $26 \%(12)$ \\
$50-59$ ans & $17 \%(8)$ \\
$\geq 60$ ans & \\
Sexe $\quad$ hommes & $63 \%(29)$ \\
femmes & $37 \%(17)$ \\
Ancienneté d'exercice & $20 \%(9)$ \\
$\quad<$ ans & $17 \%(8)$ \\
$5-9$ ans & $20 \%(9)$ \\
$10-19$ ans & $43 \%(20)$ \\
$\geq 20$ ans & $11 \%(5)$ \\
Lieu d'exercice & $35 \%(16)$ \\
Libéral & $30 \%(14)$ \\
CHU Nancy & $13 \%(6)$ \\
CHR & $11 \%(5)$ \\
Etablissement privé & \\
Activité mixte & $78 \%(36)$ \\
Spécificités d'activité (plusieurs réponses possibles) & $48 \%(22)$ \\
BPCO & $72 \%(33)$ \\
Fibrose pulmonaire & $22 \%(10)$ \\
Oncologie thoracique & $20 \%(9)$ \\
HTAP & $28 \%(13)$ \\
Pathologies neuromusculaires &
\end{tabular}

\section{3.2.2 Interventions non pharmacologiques à visée eupnéisante chez les patients}

atteints de BPCO : réponses au questionnaire

Le recours aux interventions non pharmacologiques à visée eupnéisante par les 46 médecins répondeurs est représenté Tableau $6 \mathrm{a}$. 
Tableau 6a. Recours aux interventions non pharmacologiques à visée eupnéisante par les 46 médecins répondeurs.

\begin{tabular}{l|c}
\hline Interventions non pharmacologiques & $\mathbf{\%}(\mathbf{n})$ \\
\hline RR & $96 \%(44)$ \\
Pratique sportive & $59 \%(27)$ \\
Réentrainement musculaire inspiratoire & $33 \%(15)$ \\
Relaxation & $33 \%(15)$ \\
Psychothérapie & $24 \%(11)$ \\
Yoga & $22 \%(10)$ \\
Hypnose médicale & $17 \%(8)$ \\
Sophrologie & $11 \%(5)$ \\
Distraction auditive & $2 \%(1)$ \\
Thérapie de pleine conscience & $2 \%(1)$ \\
Acupuncture & $2 \%(1)$ \\
Vibration paroi thoracique & 0 \\
Aucune & 0 \\
\hline Fréquence de prescription des interventions non pharmacologiques & \\
$\quad$ Souvent & $41 \%(19)$ \\
Parfois & $48 \%(22)$ \\
$\quad$ Rarement & $11 \%(5)$ \\
$\quad$ Jamais & 0 \\
\hline
\end{tabular}

\section{3.2.3 Traitement pharmacologique symptomatique de la dyspnée chez les patients} atteints de BPCO : réponses au questionnaire

- Traitement pharmacologique symptomatique de la dyspnée en fonction de la situation clinique :

Le recours aux traitements pharmacologiques à visée eupnéisante en fonction de la situation clinique par les 46 médecins répondeurs est représenté Tableau 2a. Les morphiniques par voie systémique sont le traitement le plus déclaré par les médecins répondeurs, particulièrement en situation palliative à $91 \%$. 
Tableau 2a. Recours aux traitements pharmacologiques symptomatiques en fonction de la situation clinique par les 46 médecins répondeurs.

\begin{tabular}{l|c|c|c}
\hline Traitements pharmacologiques à visée eupnéisante & $\begin{array}{c}\text { SITUATION } \\
\text { AMBULATOIRE }\end{array}$ & $\begin{array}{c}\text { SITUATION } \\
\text { HOSPITALIERE }\end{array}$ & $\begin{array}{c}\text { SITUATION } \\
\text { PALLIATIVE }\end{array}$ \\
\hline Morphiniques systémiques & $24 \%$ & $48 \%$ & $91 \%$ \\
Morphiniques en nébulisation & - & $4 \%$ & $20 \%$ \\
BZD & $13 \%$ & $46 \%$ & $70 \%$ \\
Oxygène en absence d'hypoxémie sévère & $17 \%$ & $30 \%$ & $52 \%$ \\
Furosémide inhalé & - & $4 \%$ & - \\
Aucun & $59 \%$ & - & - \\
\hline
\end{tabular}

Le recours aux traitements pharmacologiques à visée eupnéisante en fonction de la situation clinique et de l'ancienneté d'exercice est représenté Tableau 3a.

Tableau 3a. Recours aux traitements pharmacologiques symptomatiques à visée eupnéisante en fonction de la situation clinique et de l'ancienneté d'exercice.

\begin{tabular}{|c|c|c|c|c|c|c|}
\hline \multirow{2}{*}{$\begin{array}{l}\text { Traitements pharmacologiques à visée } \\
\text { eupnéisante }\end{array}$} & \multicolumn{2}{|c|}{$\begin{array}{c}\text { SITUATION } \\
\text { AMBULATOIRE }\end{array}$} & \multicolumn{2}{|c|}{ SITUATION HOSPITALIERE } & \multicolumn{2}{|c|}{ SITUATION PALLIATIVE } \\
\hline & $\begin{array}{c}>10 \text { ans } \\
n=29\end{array}$ & $\begin{array}{c}<10 \text { ans } \\
\mathbf{n}=17\end{array}$ & $\begin{array}{c}>10 \text { ans } \\
n=29\end{array}$ & $\begin{array}{c}<10 \text { ans } \\
\mathrm{n}=17\end{array}$ & $\begin{array}{c}>10 \text { ans } \\
n=29\end{array}$ & $\begin{array}{c}<10 \text { ans } \\
\mathbf{n}=17\end{array}$ \\
\hline Non concernés & $3,4 \%(1)$ & $5,9 \%(1)$ & $17,2 \%(5)$ & $35,3 \%(6)$ & $6,9 \%(2)$ & $11,8 \%(2)$ \\
\hline Morphiniques systémiques & $20,7 \%(6)$ & $29,4 \%(5)$ & $55,2 \%(16)$ & $35,3 \%(6)$ & $93,1 \%(27)$ & $88,2 \%(15)$ \\
\hline Morphiniques nébulisation & 0 & 0 & $3,4 \%(1)$ & $5,9 \%(1)$ & $24,1 \%(7)$ & $11,8 \%(2)$ \\
\hline BZD & $20,7 \%(6)$ & 0 & $48,3 \%(14)$ & $41,2 \%(7)$ & $72,4 \%(21)$ & $64,7 \%(11)$ \\
\hline Oxygène en absence d'hypoxémie sévère & $24,1 \%(7)$ & $5,9 \%(1)$ & $34,5 \%(10)$ & $23,5 \%(4)$ & $55,2 \%(16)$ & 0 \\
\hline Furosémide inhalé & 0 & 0 & $6,9 \%(2)$ & 0 & 0 & 0 \\
\hline Aucun & $58,6 \%(17)$ & $58,8 \%(10)$ & 0 & 0 & 0 & 0 \\
\hline
\end{tabular}

Le recours aux traitements pharmacologiques à visée eupnéisante en fonction de la situation clinique et du statut médecin inclueur ou non inclueur au questionnaire iBPCO est représenté Tableau 4a. 
Tableau 4a. Recours aux traitements pharmacologiques en fonction de la situation clinique et du statut médecin inclueur ou non inclueur.

\begin{tabular}{|c|c|c|c|c|c|c|}
\hline \multirow[b]{2}{*}{$\begin{array}{l}\text { Traitements pharmacologiques à visée } \\
\text { eupnéisante }\end{array}$} & \multicolumn{2}{|c|}{$\begin{array}{l}\text { SITUATION } \\
\text { AMBULATOIRE }\end{array}$} & \multicolumn{2}{|c|}{$\begin{array}{l}\text { SITUATION } \\
\text { HOSPITALIERE }\end{array}$} & \multicolumn{2}{|c|}{$\begin{array}{l}\text { SITUATION } \\
\text { PALLIATIVE }\end{array}$} \\
\hline & $\begin{array}{c}\text { Non } \\
\text { inclueurs } \\
\mathbf{n = 3 8}\end{array}$ & $\begin{array}{c}\text { Inclueurs } \\
\mathbf{n}=\mathbf{8}\end{array}$ & $\begin{array}{c}\text { Non } \\
\text { inclueurs } \\
\mathbf{n}=\mathbf{3 8}\end{array}$ & $\begin{array}{l}\text { Inclueurs } \\
\quad \mathbf{n}=\mathbf{8}\end{array}$ & $\begin{array}{c}\text { Non } \\
\text { inclueurs } \\
\mathbf{n}=\mathbf{3 8}\end{array}$ & $\begin{array}{l}\text { Inclueurs } \\
\qquad \mathrm{n}=\mathbf{8}\end{array}$ \\
\hline Non concernés & $5,3 \%(2)$ & 0 & $28,9 \%(11)$ & 0 & $10,5 \%(4)$ & 0 \\
\hline Morphiniques systémiques & $15,8 \%(6)$ & $62,5 \%(5)$ & $39,5 \%(15)$ & $87,5 \%(7)$ & $89,5 \%(34)$ & $100 \%(8)$ \\
\hline Morphiniques nébulisation & 0 & 0 & $2,6 \%(1)$ & $12,5 \%(1)$ & $21,1 \%(8)$ & $12,5 \%(1)$ \\
\hline BZD & $13,2 \%(5)$ & $12,5 \%(1)$ & $44,7 \%(17)$ & $50 \%(4)$ & $65,8 \%(25)$ & $87,5 \%(7)$ \\
\hline Oxygène en absence d'hypoxémie sévère & $13,2 \%(5)$ & $37,5 \%(3)$ & $26,3 \%(10)$ & $50 \%(4)$ & $50 \%(19)$ & $62,5 \%(5)$ \\
\hline Furosémide inhalé & 0 & 0 & $5,3 \%(2)$ & 0 & 0 & 0 \\
\hline Aucun & $63,2 \%(24)$ & $37,5 \%$ & 0 & 0 & 0 & 0 \\
\hline
\end{tabular}

- Recours aux morphiniques et aux BZD à visée eupnéisante :

Parmi les 46 médecins répondeurs, on retrouve 30\% des praticiens (14 médecins) réticents à l'utilisation de morphiniques et 48\% (22 médecins) réticents au recours aux BZD à visée eupnéisante pour les patients atteints de BPCO.

Les proportions de médecins réticents à la prescription de morphiniques et de BZD à visée eupnéisante pour les patients atteints de BPCO, en fonction de l'ancienneté d'exercice, du statut inclueur ou non et du secteur d'activité sont représentées Tableau 5a.

Tableau 5a. Proportions de médecins réticents à la prescription de morphiniques et de BZD à visée eupnéisante en fonction de l'ancienneté d'exercice, du statut inclueur ou non et du secteur d'activité

\begin{tabular}{|c|c|c|c|c|c|c|}
\hline \multirow{2}{*}{ Réticence à la prescription } & \multicolumn{2}{|c|}{ ANCIENNETE D'EXERCICE } & \multicolumn{2}{|c|}{$\begin{array}{l}\text { STATUT INCLUEUR NON } \\
\text { INCLUEUR }\end{array}$} & \multicolumn{2}{|c|}{ SECTEUR D'ACTIVITE } \\
\hline & $\begin{array}{l}>10 \text { ans } \\
n=29\end{array}$ & $\begin{array}{c}<10 \text { ans } \\
\mathbf{n}=17\end{array}$ & $\begin{array}{c}\text { Non inclueurs } \\
\mathbf{n}=\mathbf{3 8}\end{array}$ & $\begin{array}{c}\text { Inclueurs } \\
\mathrm{n}=\mathbf{8}\end{array}$ & $\begin{array}{c}\text { Hôpital } \\
n=36\end{array}$ & $\begin{array}{c}\text { Libéral } \\
n=5\end{array}$ \\
\hline Morphiniques systémiques & $17 \%(5)$ & $53 \%(9)$ & $32 \%(12)$ & $25 \%(2)$ & $25 \%(9)$ & $60 \%(3)$ \\
\hline BZD & $38 \%(11)$ & $71 \%(12)$ & $53 \%(20)$ & $38 \%(3)$ & $47 \%(17)$ & $80 \%(4)$ \\
\hline
\end{tabular}


Les raisons de ces réticences concernant les 46 médecins répondeurs sont répertoriées dans le Tableau 6a.

Tableau 6a. Motifs de réticence à la prescription de morphiniques et de BZD à visée eupnéisante pour les patients atteints de BPCO.

\begin{tabular}{l|c|c}
\hline Motifs de réticence à la prescription & MORPHINIQUES & BENZODIAZEPINES \\
\hline Dépression respiratoire & $66 \%$ & $74 \%$ \\
Somnolence & $53 \%$ & $35 \%$ \\
Effets secondaires digestifs & $26 \%$ & - \\
Absence d'AMM & $47 \%$ & $40 \%$ \\
Absence de recommandation & $40 \%$ & $30 \%$ \\
Absence d'efficacité & $13 \%$ & $9 \%$ \\
\hline
\end{tabular}

Pour $80 \%$ des répondeurs, la prise en charge de la dyspnée dépend de la pathologie sousjacente. 


\section{4 DISCUSSION}

\section{4.1 RESULTATS PRINCIPAUX}

La population étudiée via notre cohorte est représentative de la population française de patients atteints de BPCO (69).

La population de médecins répondeurs est représentative de la population des pneumologues de la région Lorraine avec une majorité de médecins ayant une ancienneté d'exercice élevée, exerçant préférentiellement en milieu hospitalier.

Comme dans l'étude de Betsall et al. (3) on retrouve une corrélation positive entre la dyspnée évaluée par l'échelle mMRC et la distance parcourue au test de marche de 6 minutes, le score HAD Anxiété et Dépression, le questionnaire ADL et le score SGRQ activité. Dans cette étude, il existait un lien entre la sévérité de la dyspnée et l'âge, que nous ne retrouvons pas. En revanche, notre étude objective une corrélation entre l'élévation de la capnie et la dyspnée. Selon l'intensité de la dyspnée, on distingue une population plus sévère tant sur le plan du retentissement fonctionnel (échelles de qualité de vie), gazométrique (hypoventilation alvéolaire) et spirométrique. Ces paramètres cliniques et fonctionnels sont altérés chez les patients dyspnéiques les plus sévères, c'est pourquoi nous avons souhaité étudier ce sousgroupe de population.

\section{4.1.1 Evaluation de la dyspnée}

Concernant l'évaluation de la dyspnée, il existe des échelles qui prennent en compte le retentissement de la dyspnée sur les activités de la vie quotidienne et sur la qualité de vie des 
patients, permettant d'en apprécier l'aspect multidimensionnel, comme le score Dyspnea 12 et le MDP. Dans une récente étude (70), obstruction bronchique, prévalence des symptômes dépressifs et activité physique sont déterminants dans la dimension affective de la dyspnée. Cette composante affective serait plus marquée chez les patients atteints de BPCO avec un score mMRC, HAD et un stade GOLD plus sévère.

Dans notre étude, l'évaluation de la dyspnée est unidimensionnelle via l'échelle mMRC. Cela reflète l'évaluation de la dyspnée en routine clinique qui ne prend pas en compte la part multidimensionnelle de la dyspnée : les questionnaires sont longs et fastidieux. Ainsi, dans la cohorte iBPCO, on remarque que les questionnaires longs et compliqués ne sont pas remplis en totalité par les malades. Par exemple, seuls $17 \%$ des patients ont renseigné complètement le questionnaire St Georges (SGRQ) qui comporte 17 items. L'adaptation de ces questionnaires fondamentaux à la pratique courante, au temps de consultation et à la compréhension des patients mérite d'être étudiée.

La dyspnée est un des maitres symptômes de la BPCO. L'appréciation de la dyspnée par le médecin est perturbée par sa subjectivité et sa complexité (39). En effet, la récente étude de Celli et al. (71) retrouve d'importantes différences entre la perception des patients atteints de BPCO concernant différents domaines de leur maladie et la perception qu'ont les médecins de leurs patients et de la BPCO, notamment concernant la qualité de vie et les symptômes. Il est mis en évidence également un manque de communication important entre soignants et soignés, particulièrement de la part des patients envers leur praticien concernant leurs symptômes. Cependant de nombreuses études de type enquêtes auprès des praticiens (72-75), mettent en évidence une volonté de la part des médecins de mieux prendre en charge la dyspnée. 


\section{4.1.2 Dyspnée et comorbidités}

Les relations entre dyspnée et comorbidités sont complexes. En effet, le symptôme dyspnée n'est pas spécifique de la maladie BPCO ici étudiée, de plus les comorbidités de la BPCO sont elles aussi pourvoyeuses de dyspnée. Les comorbidités, notamment cardiovasculaires, sont fréquentes dans notre cohorte (HTA 50\%, troubles du rythme $20 \%$ et insuffisance cardiaque 16\%), de même que l'obésité (43\%). L'étude en «vraie vie » permet d'étudier ces comorbidités. Dans notre étude on ne retrouve étonnamment pas de différence significative concernant les comorbidités entre les patients ayant une dyspnée sévère ou non sévère, ni entre les patients ayant une dyspnée réfractaire ou non réfractaire.

L'évaluation des patients atteints de BPCO doit néanmoins comporter l'étude des comorbidités. Cette évaluation guide les choix thérapeutiques concernant non seulement la pathologie respiratoire mais aussi les comorbidités (76).

L'ostéoporose est une des comorbidités fréquente de la BPCO avec une prévalence moyenne de 35\% (77). Dans le cadre de la BPCO, la sévérité de l'obstruction bronchique et la carence en vitamine $\mathrm{D}$ participent à la physiopathologie de l'ostéoporose. Chez ces patients, les facteurs de risque de développer une ostéoporose sont l'âge, le tabagisme, la maigreur, le recours aux traitements par CSI et l'absence d'activité physique (78). Dans notre étude, les patients du groupe dyspnée sévère ont plus d'ostéoporose que les patients du groupe non sévère $(p=0.006)$. Cela pourrait s'expliquer par la sévérité de l'obstruction bronchique, la dyspnée évaluée par l'échelle mMRC étant corrélée au degré d'obstruction bronchique, et par la sédentarité. En effet, les patients ayant une dyspnée sévère ont un handicap important avec diminution des activités physiques, ce qui représente un facteur de risque d'ostéoporose. 


\section{4.1.3 BPCO, dyspnée et symptômes anxio-dépressif (SAD)}

La prévalence du syndrome anxio-dépressif dans la population générale varie de 4 à 7\% (79), elle atteint environ $30 \%$ chez les patients atteints de BPCO (9) et reste sous diagnostiquée et de fait sous-traitée probablement par manque de données disponibles dans la littérature afin de guider cette prise en charge (80). Le score Hospital Anxiety and Depression (HAD), utilisé pour détecter les symptômes des troubles anxieux et dépressifs des patients, est validé dans la BPCO (10). Les patients atteints de BPCO et de SAD peuvent avoir une perception accrue de la dyspnée (81), alors que la dyspnée peut elle-même provoquer une souffrance psychologique de type anxiété et dépression. Le syndrome anxio-dépressif est reconnu comme une comorbidité de la BPCO qui impacte sur l'activité quotidienne, l'adhésion au traitement, la fréquence des exacerbations de BPCO, la fréquence des hospitalisations et la mortalité $(82,83)$. Il existe un lien entre la sévérité de la BPCO selon l'index BODE et la prévalence du syndrome anxio-dépressif (84). Dans notre étude, il existe une corrélation positive entre la dyspnée selon le stade mMRC et le score HAD anxiété et dépression. Et, les patients qui présentent une dyspnée sévère souffrent significativement plus de SAD (score HAD anxiété et/ou dépression >10) que les patients dyspnéiques non sévères (respectivement $\mathrm{p}=0,003$ et $\mathrm{p}=0,001$ ). Cependant, les $\mathrm{SAD}$ sont insuffisamment pris en charge. Ainsi, dans notre étude, parmi les 120 patients inclus, 28 patients (23\%) présentent un score HAD anxiété positif et 23 patients (19\%) un score HAD dépression positif, en faveur de SAD. Parmi les 64 patients souffrant de dyspnée sévère, cette proportion s'élève à $34,5 \%$ pour le score HAD anxiété et à $30 \%$ pour le score HAD dépression. Le traitement des SAD à prédominance « anxiété » par anxiolytiques concerne seulement un quart des patients dyspnéiques sévères et réfractaires. Concernant les $\mathrm{SAD}$ à prédominance «dépression» $15 \%$ des patients dyspnéiques sévères et réfractaires au score HAD dépression positif sont traités par antidépresseur. Nous ne disposons pas de donnée concernant la prise en charge 
complémentaire des SAD par suivi psychologique. Peu d'études sont disponibles concernant le traitement des SAD dans le cadre de la BPCO. La réduction de ces troubles par les traitements sont de niveau modéré pour la RR et très faible pour les thérapies cognitivocomportementales et pour les antidépresseurs (12).

Les SAD devraient être évalués chez tous les patients BPCO compte tenus des liens étroits entre les deux pathologies (12). Il semble donc nécessaire de rechercher systématiquement des arguments en faveur de SAD chez les patients atteints de BPCO au minimum par un entretien clinique, a fortiori si ceux-ci souffrent d'une dyspnée sévère, et de le traiter le cas échéant. De nouveaux essais cliniques concernant la prise en charge des SAD spécifiquement dans le cadre de la BPCO seraient souhaitables afin de clarifier la conduite à tenir thérapeutique, d'autant que notre enquête montre une réticence à la prescription de BZD par près de la moitié des pneumologues répondeurs.

\section{4.1.4 Recours aux interventions non pharmacologiques}

Selon les recommandations (12), la prise en charge non pharmacologique de la dyspnée des patients atteints de BPCO est la RR et la pratique sportive. La RR a pour objectif de réduire les symptômes tels que la dyspnée. Dans notre étude $41 \%$ des patients ayant une dyspnée sévère ou réfractaire ont bénéficié de RR. Même si ce résultat est meilleur que celui de la moyenne nationale estimée à 30\%, il reste insuffisant. De plus, les raisons de l'absence de RR ne sont pas connues. Parmi les interventions non pharmacologiques à visée eupnéisante proposées dans notre questionnaire, la RR occupe la première place. Le recours aux autres interventions est rare. Les prescriptions semblent être influencées par les moyens à disposition dans le centre dans lequel le médecin prescripteur exerce : la disponibilité de ces interventions diffère selon les structures pneumologiques et représente sans doute un frein à leur recours. 


\section{4.1.5 Traitement pharmacologique étiologique de la BPCO par bronchodilatateurs des patients atteints de dyspnée sévère et réfractaire}

Dans notre étude, le recours aux traitements pharmacologiques de la BPCO par doublet de bronchodilatateurs selon les recommandations de la SPLF (12) est satisfaisant. En effet, 94\% des patients qui souffrent d'une dyspnée sévère ont un traitement pharmacologique par bronchodilatateurs optimal. Et, tous les patients qui présentent une dyspnée réfractaire ont également, par définition, un traitement pharmacologique étiologique optimal. La dyspnée réfractaire est en effet définie par une dyspnée sévère qui persiste malgré un traitement optimal de la pathologie sous-jacente, durant plus de 3 mois, avec une intensité importante (44). Ainsi, chez ces patients qui ont une dyspnée réfractaire, un traitement symptomatique par morphinique peut être proposé à visée eupnéisante, hors AMM.

\section{4.1.6 Recours aux morphiniques à visée eupnéisante}

Concernant l'utilisation des morphiniques en pratique dans le cadre de la dyspnée, il est démontré que les morphiniques par voie systémique sont efficaces à faible dose, sans nécessité de dépasser $30 \mathrm{mg}$ par jour. En effet, la majorité des patients (70\%) est soulagée par une dose de $10 \mathrm{mg}$ par jour (85). Le traitement par morphinique à visée eupnéisante est actuellement hors AMM. La voie inhalée pour les morphiniques à visée eupnéisante n'est pas retenue $(59,60)$.

Les patients qui ne répondent pas à ce traitement ne sont pas ceux qui souffrent d'une dyspnée plus sévère, la réponse n'est pas non plus liée à la pathologie, en revanche on suggère la possible susceptibilité génétique chez les patients non répondeurs (85). Certains auteurs émettent cependant des réserves à l'utilisation des morphiniques chez le sujet 
âgé atteint de BPCO notamment, mettant en évidence la recrudescence d'effets indésirables $(86,87)$.

D'après les réponses au questionnaire adressé aux pneumologues de Lorraine, la prescription de morphinique à visée eupnéisante dans le cadre de la BPCO varie en fonction de la structure de prescription ( $24 \%$ en ambulatoire versus $48 \%$ en situation hospitalière) et ce quelle que soit l'ancienneté d'exercice. Le recours aux morphiniques augmente également au fur et à mesure que la maladie s'aggrave et ce quelle que soit l'ancienneté d'exercice. Parmi les 46 médecins répondeurs, 91\% des médecins déclarent prescrire de la morphinique dans le cadre des soins palliatifs versus $24 \%$ en ambulatoire, cette tendance se retrouve également dans l'étude anglaise de Hadjiphilippou et al. (72). Cependant la prescription de morphiniques dans le cadre de la BPCO au stade terminal est moindre qu'au cours d'un cancer bronchique de stade palliatif (88). Dans notre étude parmi les 64 patients qui ont une dyspnée sévère, dont 41 patients souffrant d'une dyspnée réfractaire, aucun patient ne bénéficie d'un traitement par morphiniques systémiques.

La morphine est de façon générale peu prescrite pour soulager la dyspnée réfractaire (50), possiblement par crainte des effets indésirables respiratoires (62,72-75). Cependant, la morphine prescrite à de faibles doses n'entraînerait pas d'effet secondaire respiratoire tels qu'une insuffisance respiratoire aiguë hypercapnique ou une surmortalité, y compris chez des patients atteints de BPCO sévère ou au stade d'insuffisance respiratoire terminale (62). La récente revue de la littérature Cochrane (89) concernant le recours aux morphiniques à visée eupnéisante n'a pas retrouvé de modification significative de la gazométrie artérielle en faveur d'une hypoventilation alvéolaire secondaire. On note en revanche des effets secondaires d'ordre digestif à type de nausées, vomissements et constipation, ou neurologiques avec confusion et vertiges $(59,60,85)$. Dans notre étude, parmi les 46 médecins répondeurs, 30\% expriment une réticence à la 
prescription de morphiniques à visée eupnéisante dans le cadre d'une BPCO. Soixante-six pour cent d'entre eux justifient cette réticence par crainte des effets secondaires respiratoires à type de dépression respiratoire. Cette proportion est retrouvée dans la littérature au sein de plusieurs études de petits effectifs et concerne environ 55\% des médecins interrogés $(72,75)$. Cependant, dans l'étude de Janssen et al. de 2015 (73), 20\% des 146 pneumologues interrogés décrivaient une réserve à la prescription de morphiniques à visée eupnéisante. Une récente étude publiée en 2017 (74) concernant l'évaluation des pratiques des jeunes pneumologues australiens retrouve également une proportion moindre à $25 \%$. Dans notre étude, les jeunes médecins semblent, au contraire, plus réticents que leurs aînés à la prescription de morphiniques dans ce contexte.

Par ailleurs, deux études $(73,75)$ développent la notion de «morphinophobie » exprimée par les patients comme un frein à la prescription de morphiniques à visée eupnéisante. Cette notion n'a pas été étudiée dans notre questionnaire.

Dans l'étude de Young et al. (75), les médecins interrogés reportaient un manque de connaissance, de formation et de recommandations concernant la prescription de morphiniques dans ce contexte. Dans notre étude, 40\% des médecins réticents à l'utilisation de morphiniques le justifient par manque de recommandations. Un essai de phase 3 est actuellement en cours (90), les résultats de cette étude permettront une avancée concernant le recours aux morphiniques à visée eupnéisante.

Le recours aux BZD à visée eupnéisante ne fait pas l'objet de recommandations spécifiques. 


\section{4.2 FORCES ET LIMITES}

Notre étude est un travail prospectif via la cohorte iBPCO. Il s'agit d'une cohorte multicentrique, utilisant de nombreux scores validés dans le cadre de la BPCO. Le questionnaire a permis d'interroger les pneumologues de la région Lorraine, quelque soit leur mode et leur spécificité d'exercice. Nous avons recueilli un nombre satisfaisant de réponses $(52 \%)$.

Cette étude se décline en deux parties complémentaires. En effet, les pratiques professionnelles ont pu être objectivement décrites via la cohorte iBPCO et ces pratiques ont été explicitées grâce au questionnaire.

Le caractère transversal de l'étude est un facteur limitant.

Nous ne disposons pas de données suffisantes concernant la distension pulmonaire. La deuxième partie de notre étude résulte d'une enquête auprès des pneumologues de Lorraine concernant la prise en charge de la dyspnée chez les patients atteints de BPCO. Le caractère déclaratif de cette enquête représente une limite à notre étude, comme en témoignent les discordances entre la prise en charge des patients de la cohorte iBPCO et les réponses au questionnaire. Il faut néanmoins différencier intention de prescription et faisabilité clinique. Par exemple, 96\% des praticiens disent avoir recours à la RR or seulement $34 \%$ des patients de la cohorte en ont bénéficié. Cela peut s'expliquer par de nombreux obstacles à la réalisation de la RR comme les difficultés d'accès à la RR (faible disponibilité des kinésithérapeutes, manque de structures), les douleurs chroniques, le manque de motivation ou le refus du patient. Les raisons de non la réalisation de la RR ne sont pas connues ici. 


\section{5 CONCLUSION}

La dyspnée est un symptôme important de la BPCO, elle est souvent multifactorielle. D'après notre étude, la sévérité de la dyspnée ou son caractère réfractaire semblent associés à la sévérité de l'obstruction bronchique et à la présence de symptômes anxio-dépressifs plus nombreux. En revanche, il n'existe pas de lien entre la sévérité de la dyspnée et l'existence de comorbidités.

La prise en charge non pharmacologique du patient atteint de BPCO par la RR, notamment en cas de dyspnée sévère ou réfractaire, est satisfaisante.

Concernant la prise en charge pharmacologique étiologique du patient atteint de BPCO avec dyspnée sévère, un traitement optimal selon les recommandations de la SPLF 2009 est délivré à la quasi-totalité des patients. En revanche, aucun patient avec dyspnée sévère et/ou réfractaire n'est traité par morphinique à visée eupnéisante. Le recours aux morphiniques, bien que connu des pneumologues semble dépendre de la structure de prescription et de la gravité de la pathologie sous-jacente, et ce quelle que soit l'ancienneté d'exercice.

Notre étude met en exergue l'intérêt d'une plus large communication concernant la dyspnée dans le cadre de la BPCO, ses liens étroits avec les symptômes anxio-dépressifs et sa prise en charge pharmacologique symptomatique par la morphine.

Cohorte iBPCO avec le soutien de Boeringher Ingelheim France 


\section{BIBLIOGRAPHIE :}

1. Vestbo J, Hurd SS, Agustí AG, Jones PW, Vogelmeier C, Anzueto A, et al. Global Strategy for the Diagnosis, Management, and Prevention of Chronic Obstructive Pulmonary Disease: GOLD Executive Summary. Am J Respir Crit Care Med. 15 févr 2013;187(4):347-65.

2. Global initiative for chronic obstructive lung disease (GOLD). Global strategy for the diagnosis, management and prevention of COPD (2017 report). Disponible sur : http://www.goldcopd.org.

3. Bestall J, Paul E, Garrod R, Garnham R, Jones P, Wedzicha J. Usefulness of the Medical Research Council (MRC) dyspnoea scale as a measure of disability in patients with chronic obstructive pulmonary disease. Thorax. juill 1999;54(7):581-6.

4. Eisner MD, Anthonisen N, Coultas D, Kuenzli N, Perez-Padilla R, Postma D, et al. An official American Thoracic Society public policy statement: Novel risk factors and the global burden of chronic obstructive pulmonary disease. Am J Respir Crit Care Med. 1 sept 2010;182(5):693-718.

5. Charlson M, Charlson RE, Briggs W, Hollenberg J. Can Disease Management Target Patients Most Likely to Generate High Costs? The Impact of Comorbidity. J Gen Intern Med. avr 2007;22(4):464-9.

6. Burgel P-R, Escamilla R, Perez T, Carré P, Caillaud D, Chanez P, et al. Impact of comorbidities on COPD-specific health-related quality of life. Respir Med. févr 2013;107(2):233-41.

7. Sin DD, Wu L, Man SP. The relationship between reduced lung function and cardiovascular mortality: a population-based study and a systematic review of the literature. Chest. 2005;127(6):1952-1959.

8. Burgel D, Burgel P-R. Rôle des comorbidités dans l'évolution de la BPCO. Rev Mal Respir. 1 nov 2008;25(9):11-5.

9. Bock K, Bendstrup E, Hilberg O, Løkke A. Screening tools for evaluation of depression in Chronic Obstructive Pulmonary Disease (COPD). A systematic review. Eur Clin Respir J. 12 juin 2017 [cité 29 juill 2017];4(1).

10. Zigmond AS, Snaith RP. The Hospital Anxiety and Depression Scale. Acta Psychiatr Scand. juin 1983;67(6):361-70.

11. Cuvelier A. Facteurs pronostiques de la BPCO: du VEMS à l'index BODE. Rev Mal Respir. 2004;21(5):17-19. 
12. Recommandations de la Société de Pneumologie de Langue Française sur la prise en charge de la BPCO (mise à jour 2009). Presse Médicale. sept 2010;39(9):895-8.

13. Anthonisen NR, Wright EC, Hodgkin JE. Prognosis in chronic obstructive pulmonary disease. Am Rev Respir Dis. janv 1986;133(1):14-20.

14. Groenewegen KH, Schols AM, Wouters EF. Mortality and mortality-related factors after hospitalization for acute exacerbation of COPD. CHEST J. 2003;124(2):459-467.

15. Nichimura K, Izumi T, Tsukino M, Oga T. Dyspnea is a better predictor of 5-year survival than airway obstruction in patients with COPD. Chest. 2002;121(5):1434.

16. Chailleux E, Laaban J-P, Veale D. Prognostic value of nutritional depletion in patients with COPD treated by long-term oxygen therapy: data from the ANTADIR observatory. Chest. mai 2003;123(5):1460-6.

17. Marti S. Body weight and comorbidity predict mortality in COPD patients treated with oxygen therapy. Eur Respir J. 2 févr 2006;27(4):689-96.

18. Vestbo J, Prescott E, Almdal T, Dahl M, Nordestgaard BG, Andersen T, et al. Body mass, fat-free body mass, and prognosis in patients with chronic obstructive pulmonary disease from a random population sample: findings from the Copenhagen City Heart Study. Am J Respir Crit Care Med. 1 janv 2006;173(1):79-83.

19. Celli BR, Cote CG, Marin JM, Casanova C, Montes de Oca M, Mendez RA, et al. The body-mass index, airflow obstruction, dyspnea, and exercise capacity index in chronic obstructive pulmonary disease. N Engl J Med. 2004;350(10):1005-1012.

20. Rodriguez-Roisin R. Toward a consensus definition for COPD exacerbations. Chest. mai 2000;117:398S-401S.

21. Papi A, Luppi F, Franco F, Fabbri LM. Pathophysiology of exacerbations of chronic obstructive pulmonary disease. Proc Am Thorac Soc. mai 2006;3(3):245-51.

22. Fuhrman C, Delmas M-C, pour le groupe épidémiologie et recherche clinique de la SPLF. Epidemiology of chronic obstructive pulmonary disease in France. Rev Mal Respir. févr 2010;27(2):160-8.

23. WHO | Chronic obstructive pulmonary disease (COPD) [Internet]. WHO. [cité 6 août 2017]. Disponible sur: http://www.who.int/mediacentre/factsheets/fs315/en/

24. Mathers CD, Loncar D. Projections of global mortality and burden of disease from 2002 to 2030. Plos Med. 2006;3(11):e442.

25. Barnes PJ, Shapiro SD, Pauwels RA. Chronic obstructive pulmonary disease: molecular and cellularmechanisms. Eur Respir J. oct 2003;22(4):672-88. 
26. Physiopathologie des BPCO - EM|consulte [Internet]. [cité 18 avr 2017]. Disponible sur: http://www.em-consulte.com/rmr/article/157181

27. Gan W, Man S, Senthilselvan A, Sin D. Association between chronic obstructive pulmonary disease and systemic inflammation: a systematic review and a meta-analysis. Thorax. juill 2004;59(7):574-80.

28. Anthonisen NR, Skeans MA, Wise RA, Manfreda J, Kanner RE, Connett JE, et al. The effects of a smoking cessation intervention on 14.5-year mortality: a randomized clinical trial. Ann Intern Med. 15 févr 2005;142(4):233-9.

29. Scanlon PD, Connett JE, Waller LA, Altose MD, Bailey WC, Buist AS, et al. Smoking cessation and lung function in mild-to-moderate chronic obstructive pulmonary disease. The Lung Health Study. Am J Respir Crit Care Med. févr 2000;161(2 Pt 1):381-90.

30. Tonnesen P, Carrozzi L, Fagerstrom KO, Gratziou C, Jimenez-Ruiz C, Nardini S, et al. Smoking cessation in patients with respiratory diseases: a high priority, integral component of therapy. Eur Respir J. 27 sept 2006;29(2):390-417.

31. Strategies therapeutiques aide sevrage tabagique_rapport - [Internet]. [cité 20 févr 2017]. Disponible sur: http://www.has-sante.fr/portail/upload /docs/application /pdf/strategies_therapeutiques_aide_sevrage_tabagique_rapport_2007_01_22_16_28_1 4_826.pdf

32. Recommandations de la Société de Pneumologie de Langue Française sur la réhabilitation du malade atteint de BPCO - EM|consulte [Internet]. [cité 18 avr 2017]. Disponible sur: http://www.em-consulte.com/rmr/article/157152

33. Puhan MA, Scharplatz M, Troosters T, Steurer J. Respiratory rehabilitation after acute exacerbation of COPD may reduce risk for readmission and mortality - a systematic review. Respir Res. 2005;6(1):54.

34. Mahler DA, Decramer M, D'Urzo A, Worth H, White T, Alagappan VKT, et al. Dual bronchodilation with QVA149 reduces patient-reported dyspnoea in COPD: the BLAZE study. Eur Respir J. 1 juin 2014;43(6):1599-609.

35. Roche N. Position of the French Language Society of Pulmonology regarding the 2011 version of the GOLD document. Rev Mal Respir. mai 2012;29(5):637-9.

36. Zysman M, Chabot F, Devillier P, Housset B, Panzini CM, Roche N. Pharmacological treatment optimization for stable chronic obstructive pulmonary disease. Proposals from the Société de Pneumologie de Langue Française. Rev Mal

37. Long term domiciliary oxygen therapy in chronic hypoxic cor pulmonale complicating chronic bronchitis and emphysema. Report of the Medical Research Council Working Party. Lancet Lond Engl. 28 mars 1981;1(8222):681-6. 
38. Lewis CA, Fergusson W, Eaton T, Zeng I, Kolbe J. Isolated nocturnal desaturation in COPD: prevalence and impact on quality of life and sleep. Thorax. févr 2009;64(2):133-8.

39. Morelot-Panzini C, Similowski T. Dyspnée : des concepts aux thérapeutiques. Rev Mal Respir Actual. mars 2016;8(1):60-8.

40. Parshall MB, Schwartzstein RM, Adams L, Banzett RB, Manning HL, Bourbeau J, et al. An Official American Thoracic Society Statement: Update on the Mechanisms, Assessment, and Management of Dyspnea. Am J Respir Crit Care Med. 15 févr 2012;185(4):435-52.

41. Lansing RW, Im BS-H, Thwing JI, Legedza ATR, Banzett RB. The Perception of Respiratory Work and Effort Can Be Independent of the Perception of Air Hunger. Am J Respir Crit Care Med. 1 nov 2000;162(5):1690-6.

42. Lansing RW, Gracely RH, Banzett RB. the multiple dimensions of dyspnea: review and hypotheses. Respir Physiol Neurobiol. 30 mai 2009;167(1):53-60.

43. Kroenke K, Stump T, Clark DO, Callahan CM, McDonald CJ. Symptoms in hospitalized patients: outcome and satisfaction with care. Am J Med. nov 1999;107(5):425-31.

44. Wiseman R, Rowett D, Allcroft P, Abernethy A, Currow D. Chronic refractory dyspnoea: Evidence based management. Aust Fam Physician. 2013;42(3):137.

45. Widdicombe J. Lung afferent activity: implications for respiratory sensation. Respir Physiol Neurobiol. 30 mai 2009;167(1):2-8.

46. Banzett RB, Mulnier HE, Murphy K, Rosen SD, Wise RJ, Adams L. Breathlessness in humans activates insular cortex. Neuroreport. 14 juill 2000;11(10):2117-20.

47. Davenport PW, Vovk A. Cortical and subcortical central neural pathways in respiratory sensations. Respir Physiol Neurobiol. 30 mai 2009;167(1):72-86.

48. Banzett RB, O’Donnell CR, Guilfoyle TE, Parshall MB, Schwartzstein RM, Meek PM, et al. Multidimensional Dyspnea Profile: an instrument for clinical and laboratory research. Eur Respir J. 2015;ERJ-00389.

49. Yorke J, Moosavi SH, Shuldham C, Jones PW. Quantification of dyspnoea using descriptors: development and initial testing of the Dyspnoea-12. Thorax. janv 2010;65(1):21-6.

50. Janssen DJA, Spruit MA, Uszko-Lencer NH, Schols JMGA, Wouters EFM. Symptoms, comorbidities, and health care in advanced chronic obstructive pulmonary disease or chronic heart failure. J Palliat Med. juin 2011;14(6):735-43. 
51. Blinderman CD, Homel P, Andrew Billings J, Tennstedt S, Portenoy RK. Symptom Distress and Quality of Life in Patients with Advanced Chronic Obstructive Pulmonary Disease. J Pain Symptom Manage. juill 2009;38(1):115-23.

52. O'Donnell DE, Banzett RB, Carrieri-Kohlman V, Casaburi R, Davenport PW, Gandevia SC, et al. Pathophysiology of Dyspnea in Chronic Obstructive Pulmonary Disease: A Roundtable. Proc Am Thorac Soc. 1 mai 2007;4(2):145-68.

53. Rennard S, Decramer M, Calverley PMA, Pride NB, Soriano JB, Vermeire PA, et al. Impact of COPD in North America and Europe in 2000: subjects' perspective of Confronting COPD International Survey. Eur Respir J. 1 oct 2002;20(4):799-805.

54. Comment mesurer et suivre la distension pulmonaire au cours de la BPCO EM|consulte [Internet]. [cité 23 août 2017]. Disponible sur: http://www.emconsulte.com/rmr/article/212445/

55. O'Donnell DE, Flüge T, Gerken F, Hamilton A, Webb K, Aguilaniu B, et al. Effects of tiotropium on lung hyperinflation, dyspnoea and exercise tolerance in COPD. Eur Respir J. juin 2004;23(6):832-40.

56. O'Donnell DE, Voduc N, Fitzpatrick M, Webb KA. Effect of salmeterol on the ventilatory response to exercise in chronic obstructive pulmonary disease. Eur Respir J. 1 juill 2004;24(1):86-94.

57. Banzett RB, Lansing RW, Reid MB, Adams L, Brown R. « Air hunger » arising from increased PCO2 in mechanically ventilated quadriplegics. Respir Physiol. avr 1989;76(1):53-67.

58. Moosavi SH, Golestanian E, Binks AP, Lansing RW, Brown R, Banzett RB. Hypoxic and hypercapnic drives to breathe generate equivalent levels of air hunger in humans. J Appl Physiol Bethesda Md 1985. janv 2003;94(1):141-54.

59. Jennings A-L, Davies AN, Higgins JPT, Gibbs JSR, Broadley KE. A systematic review of the use of opioids in the management of dyspnoea. Thorax. 11 janv 2002;57(11):93944.

60. Ekström M, Nilsson F, Abernethy AA, Currow DC. Effects of opioids on breathlessness and exercise capacity in chronic obstructive pulmonary disease. A systematic review. Ann Am Thorac Soc. juill 2015;12(7):1079-92.

61. Banzett RB, Adams L, O'Donnell CR, Gilman SA, Lansing RW, Schwartzstein RM. Using Laboratory Models to Test Treatment. Am J Respir Crit Care Med. 15 oct 2011;184(8):920-7. 
62. Ekstrom MP, Bornefalk-Hermansson A, Abernethy AP, Currow DC. Safety of benzodiazepines and opioids in very severe respiratory disease: national prospective study. BMJ. 30 janv 2014;348(jan30 2):g445-g445.

63. Faymonville M-E, Boly M, Laureys S. Functional neuroanatomy of the hypnotic state. J Physiol Paris. juin 2006;99(4-6):463-9.

64. Moosavi SH, Binks AP, Lansing RW, Topulos GP, Banzett RB, Schwartzstein RM. Effect of inhaled furosemide on air hunger induced in healthy humans. Respir Physiol Neurobiol. 16 avr 2007;156(1):1-8.

65. Jensen D, Amjadi K, Harris-McAllister V, Webb KA, O'Donnell DE. Mechanisms of dyspnoea relief and improved exercise endurance after furosemide inhalation in COPD. Thorax. juill 2008;63(7):606-13.

66. Abernethy AP, McDonald CF, Frith PA, Clark K, Herndon JE, Marcello J, et al. Effect of palliative oxygen versus room air in relief of breathlessness in patients with refractory dyspnoea: a double-blind, randomised controlled trial. Lancet Lond Engl. 4 sept 2010;376(9743):784-93.

67. Galbraith S, Fagan P, Perkins P, Lynch A, Booth S. Does the use of a handheld fan improve chronic dyspnea? A randomized, controlled, crossover trial. J Pain Symptom Manage. mai 2010;39(5):831-8.

68. Kanezaki M, Ebihara S. Effect of the cooling sensation induced by olfactory stimulation by L-menthol on dyspnoea: a pilot study. Eur Respir J. avr 2017;49(4).

69. Agusti A, Calverley PM, Celli B, Coxson HO, Edwards LD, Lomas DA, et al. Characterisation of COPD heterogeneity in the ECLIPSE cohort. Respir Res. 2010;11(1):122.

70. Morélot-Panzini C, Gilet H, Aguilaniu B, Devillier P, Didier A, Perez T, et al. Reallife assessment of the multidimensional nature of dyspnoea in COPD outpatients. Eur Respir J. 1 juin 2016;47(6):1668-79.

71. Celli B, Blasi F, Gaga M, Singh D, Vogelmeier C, Pegoraro V, et al. Perception of symptoms and quality of life \&ndash; comparison of patients\&rsquo; and physicians\&rsquo; views in the COPD MIRROR study [Internet]. International Journal of Chronic Obstructive Pulmonary Disease. 2017 [cité 31 juill 2017].

72. Hadjiphilippou S, Odogwu S-E, Dand P. Doctors' attitudes towards prescribing opioids for refractory dyspnoea: a single-centred study. BMJ Support Palliat Care. 1 juin 2014;4(2):190-2.

73. Janssen DJ, de Hosson S, bij de Vaate E, Mooren KJ, Baas AA. Attitudes toward opioids for refractory dyspnea in COPD among Dutch chest physicians. Chron Respir Dis. mai 2015;12(2):85-92. 
74. Smallwood N, Gaffney N, Gorelik A, Irving L, Le B, Philip J. Junior doctors' attitudes to opioids for refractory breathlessness in patients with advanced chronic obstructive pulmonary disease. Intern Med J. 6 juin 2017;

75. Young J, Donahue M, Farquhar M, Simpson C, Rocker G. Using opioids to treat dyspnea in advanced COPD. Can Fam Physician. juill 2012;58(7):e401-7.

76. Burgel P-R. Des définitions aux phénotypes de BPCO. Presse Médicale. déc 2014;43(12):1337-43.

77. Inoue D, Watanabe R, Okazaki R. COPD and osteoporosis: links, risks, and treatment challenges. Int J Chron Obstruct Pulmon Dis. 29 mars 2016;11:637-48.

78. Lehouck A, Boonen S, Decramer M, Janssens W. COPD, Bone Metabolism, and Osteoporosis. Chest. mars 2011;139(3):648-57.

79. Ferrari AJ, Charlson FJ, Norman RE, Patten SB, Freedman G, Murray CJL, et al. Burden of Depressive Disorders by Country, Sex, Age, and Year: Findings from the Global Burden of Disease Study 2010. PLoS Med. 5 nov 2013 ;10(11).

80. Maurer J, Rebbapragada V, Borson S, Goldstein R, Kunik ME, Yohannes AM, et al. Anxiety and Depression in COPD. Chest. oct 2008;134(4 Suppl):43S-56S.

81. Chan P-YS, von Leupoldt A, Bradley MM, Lang PJ, Davenport PW. The effect of anxiety on respiratory sensory gating measured by respiratory-related evoked potentials. Biol Psychol. oct 2012;91(2):185-9.

82. Papaioannou AI, Bartziokas K, Tsikrika S, Karakontaki F, Kastanakis E, Banya W, et al. The impact of depressive symptoms on recovery and outcome of hospitalised COPD exacerbations. Eur Respir J. avr 2013;41(4):815-23.

83. Laviolette L, Laveneziana $\mathrm{P}$, on behalf of the ERS Research Seminar Faculty. Dyspnoea: a multidimensional and multidisciplinary approach. Eur Respir J. 1 juin 2014;43(6):1750-62.

84. Al-shair K, Dockry R, Mallia-Milanes B, Kolsum U, Singh D, Vestbo J. Depression and its relationship with poor exercise capacity, BODE index and muscle wasting in COPD. Respir Med. oct 2009;103(10):1572-9.

85. Currow DC, McDonald C, Oaten S, Kenny B, Allcroft P, Frith P, et al. Once-Daily Opioids for Chronic Dyspnea: A Dose Increment and Pharmacovigilance Study. J Pain Symptom Manage. sept 2011;42(3):388-99.

86. Vozoris NT, Wang X, Fischer HD, Bell CM, O’Donnell DE, Austin PC, et al. Incident opioid drug use and adverse respiratory outcomes among older adults with COPD. Eur Respir J. 13 juill 2016;ERJ-01967-2015. 
87. Vozoris NT, Wang X, Fischer HD, Gershon AS, Bell CM, Gill SS, et al. Incident opioid drug use among older adults with chronic obstructive pulmonary disease: a populationbased cohort study. Br J Clin Pharmacol. 1 janv 2016;81(1):161-70.

88. Gore JM, Brophy CJ, Greenstone MA. How well do we care for patients with end stage chronic obstructive pulmonary disease (COPD)? A comparison of palliative care and quality of life in COPD and lung cancer. Thorax. 2000;55(12):1000-1006.

89. Barnes H, McDonald J, Smallwood N, Manser R. Opioids for the palliation of refractory breathlessness in adults with advanced disease and terminal illness. Cochrane Database Syst Rev. 31 mars 2016;3:CD011008.

90. Currow D, Watts GJ, Johnson M, McDonald CF, Miners JO, Somogyi AA, et al. A pragmatic, phase III, multisite, double-blind, placebo-controlled, parallel-arm, dose increment randomised trial of regular, low-dose extended-release morphine for chronic breathlessness: Breathlessness, Exertion And Morphine Sulfate (BEAMS) study protocol. BMJ Open. juill 2017;7(7):e018100. 
ANNEXES 
Annexe 1 


\title{
ETUDE DE COHORTE PROSPECTIVE DYNAMIQUE MULTICENTRIQUE DES BRONCHOPNEUMOPATHIES CHRONIQUES OBSTRUCTIVES
}

\section{Document d'information et de consentement}

\author{
INFORMATION DU PATIENT
}

Madame, Mademoiselle, Monsieur,

Le pneumologue que vous consultez a diagnostiqué une maladie appelée BPCO ou bronchopneumopathie chronique obstructive.

Cette maladie se caractérise par un rétrécissement des bronches, gênant le passage de l'air. Ses symptômes sont une toux et des crachats et un essoufflement. Ils sont inconstants. La BPCO touche environ 4 à 10\% de la population adulte. C'est donc une maladie très fréquente. Elle peut devenir une source de handicap en raison de l'essoufflement qu'elle peut entraîner. Elle constitue donc un enjeu majeur de santé publique. Plusieurs traitements et mesures d'hygiène de vie peuvent limiter le risque de handicap. Néanmoins, les connaissances sur la BPCO doivent progresser pour que la prise en charge de cette maladie soit encore plus efficace. En particulier, il est nécessaire de chercher si certains groupes de malades ont des caractéristiques ou une évolution particulières qui pourraient justifier une prise en charge spécifique.

Dans ce but, une Association de médecins pneumologues (Association « Initiatives BPCO ») a mis en place une étude qui doit porter sur un grand nombre de malades (l'objectif initial est de 5000 personnes). Les malades étudiés constituent une " cohorte ». C'est pourquoi l'étude porte le nom de « cohorte prospective dynamique BPCO » ou COPD-BPCO. Elle est effectuée dans XX centres en France.

Votre pneumologue vous a proposé de faire partie de cette étude.

Que vous acceptiez ou refusiez ne changera rien à votre relation avec votre pneumologue, ni aux examens ou traitements qu'il sera éventuellement amené à vous proposer : si vous faites partie de la cohorte, votre pneumologue transmettra simplement les données vous concernant, sans mention de votre identité, à un fichier pour analyse. Ces données seront celles que votre pneumologue juge nécessaire dans le cadre de la prise en charge habituelle des patients atteints de BPCO, qu'ils fassent ou non partie de la cohorte COPD-BPCO. Parmi ces données, votre origine ethnique sera consignée, comme cela est systématiquement indispensable pour le calcul des valeurs normales des fonctions respiratoires et, donc, l'interprétation de vos résultats.

Votre participation restera anonyme : les informations recueillies sont traitées confidentiellement par informatique. Le fichier informatique utilisé a fait l'objet d'une autorisation de la CNIL (Commission Nationale de l'Informatique et des Libertés) en application des articles 40-1 et suivants de la loi "Informatique et libertés", dernière modification en date du 6 août 2004. Conformément à la loi, vous pouvez exercer votre droit d'accès auprès de votre pneumologue qui vous communiquera directement ou par l'intermédiaire d'un autre médecin de votre choix les données vous concernant. Vous pouvez également exercer votre droit de rectification auprès de votre pneumologue. Vous êtes libre de refuser de participer à cette cohorte et ceci tout au long de l'étude.

Le présent formulaire est le seul document où votre nom est mentionné. II ne sera pas transmis au centre de traitement des données. Un exemplaire vous sera remis par votre pneumologue, qui gardera l'autre dans votre dossier médical. 


\section{CONSENTEMENT}

Numéro du centre :

Numéro du patient :

En application de la loi « Informatique et libertés » du 6 janvier 1978 dernières modifications en date du 6 août 2004 par la loi 2004-801, j'accepte que les données enregistrées à l'occasion de cette étude, y compris mes données ethniques, puissent faire l'objet d'un traitement informatisé par l'Association Initiatives BPCO ou pour son compte.

J'ai bien noté que le droit d'accès (article 39 et 43) et de rectification (article 40) que m'ouvrent les textes susvisés, pourront s'exercer à tout moment auprès du $\mathrm{Dr}$ de l'Association Initiatives BPCO , 191 avenue Aristide Briand, 94234 Cachan Cedex, et que les données me concernant pourront m'être communiquées directement ou par l'intermédiaire d'un médecin de mon choix.

Je certifie avoir reçu un exemplaire original de ce formulaire de consentement daté et signé.

Je consens volontairement à participer à cette étude.

Le patient : (à remplir par le patient personnellement au moment du consentement)

Nom :

(majuscules)
Prénom :

(majuscules)

Date :

Signature :

(à remplir par le patient au moment du consentement)

\section{Le médecin :}

Je confirme avoir expliqué la nature, le but et les effets prévisibles de l'étude et la nécessite de recueillir les données ethniques au patient identifié ci-dessus.

Nom :

(majuscules)

\section{Prénom :}

(majuscules)

\section{Signature :}

Date :

L'Association Initiatives BPCO vous remercie d'avoir pris connaissance de ces éléments et, si tel est le cas, d'avoir accepté de faire partie de cette étude. 


\section{Patient :}

Nom (les 2 premieres lettres)

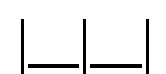

Prénom (es 3 premierese eltres)

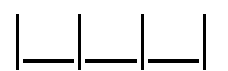

Date de la consultation :

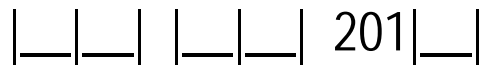

\section{COHORTE I. BPCO \\ (COPD - BPCO)}

Merci de renseigner les échelles et questionnaires contenus dans ce document

- MRC

- HAD

- St George's Respiratory Questionnaire

- ADL

- CASA-Q

- VSRQ

- EPWORTH

Et le remettre à votre médecin 


\section{ECHELLE MRC}

Echelle MRC (veuillez entourer le chiffre correspondant à votre situation) :

0 : Essoufflé seulement pour des efforts intenses

1 : Essoufflé en hâtant le pas ou en montant une légère côte

2 : Marche sur un terrain plat plus lentement que les sujets de son âge ou doit s'arrêter en marchant à son rythme sur terrain plat

3 : Doit s'arrêter après $100 \mathrm{~m}$ ou quelques minutes de marche

4 : Trop essoufflé pour sortir de la maison 
Pourquoi ces questions?

Les médecins savent que les émotions jouent un rôle important dans la plupart des maladies. Si votre médecin est au courant des émotions que vous éprouvez, il pourra mieux vous aider. Ce questionnaire a été conçu de façon à permettre à votre médecin de se familiariser avec ce que vous éprouvez vous-même sur le plan émotif.

Ne faites pas attention aux chiffres et aux lettres imprimés à gauche du questionnaire. Lisez chaque série de questions et soulignez la réponse qui exprime le mieux ce que vous avez éprouvé au cours de la semaine qui vient de s'écouler. Ne vous attardez pas sur la réponse à faire, votre réaction immédiate à chaque question fournira probablement une meilleure indication de ce que vous éprouvez, qu'une réponse longue et méditée.
A Je me sens tendu(e) ou énervé(e)
3 La plupart du temps
2 Souvent
1 De temps en temps
0 Jamais
D Je prends plaisir aux mêmes choses qu'autrefois
0 Oui, tout autant
1 Pas autant
2 Un peu seulement
3 Presque plus

A J'ai une sensation de peur comme si quelque chose d'horrible allait m'arriver

3 Oui, très nettement

2 Oui, mais ce n'est pas trop grave

1 Un peu, mais cela ne m'inquiète pas

0 Pas du tout

D Je ris facilement et vois le bon côté des choses

$0 \quad$ Autant que par le passé

1 Plus autant qu'avant

2 Vraiment moins qu'avant

3 Plus du tout

A Je me fais du souci

3 Très souvent

2 Assez souvent

1 Occasionnellement

0 Très occasionnellement

D Je suis de bonne humeur

3 Jamais

2 Rarement

1 Assez souvent

0 La plupart du temps

A Je peux rester tranquillement assis(e) à ne rien faire et me sentir décontracté(e)

0 Oui, quoi qu'il arrive

1 Oui, en général

2 Rarement

3 Jamais
D J'ai l'impression de fonctionner au ralenti

3 Presque toujours

2 Très souvent

1 Parfois

0 Jamais

A J'éprouve des sensations de peur et j'ai l'estomac noué

0 Jamais

1 Parfois

2 Assez souvent

3 Très souvent

D Je ne m'intéresse plus à mon apparence

3 Plus du tout

2 Je n'y accorde pas autant d'attention que je le devrais

1 II se peut que je n'y fasse plus autant attention

0 J'y prête autant d'attention que par le passé

A J'ai la bougeotte et n'arrive plus à tenir en place

3 Oui, c'est tout à fait le cas

2 Un peu

1 Pas tellement

0 Pas du tout

D Je me réjouis d'avance à l'idée de faire certaines choses

0 Autant qu'avant

1 Un peu moins qu'avant

2 Bien moins qu'avant

3 Presque jamais

A J'éprouve des sensations soudaines de panique

3 Vraiment très souvent

2 Assez souvent

1 Pas très souvent

0 Jamais

D Je peux prendre plaisir à un bon livre ou à une bonne émission radio ou de télévision

0 Souvent

1 Parfois

2 Rarement

3 Très rarement 


\section{1ère PARTIE}

Les questions qui suivent cherchent à déterminer l'importance des problèmes respiratoires que vous avez pu ressentir AU COURS DES 12 DERNIERS MOIS

(Mettez une croix dans la case correspondant à votre réponse à chaque question)

\begin{tabular}{ccccc}
$\begin{array}{c}\text { Presque } \\
\text { tous les }\end{array}$ & $\begin{array}{c}\text { Plusieurs } \\
\text { jours par } \\
\text { jours de la } \\
\text { semaine }\end{array}$ & $\begin{array}{c}\text { Quelques } \\
\text { jours par } \\
(2-4 \text { jours })\end{array}$ & $\begin{array}{c}\text { Seulement } \\
\text { mondant une } \\
\text { infection } \\
\text { respiratoire }\end{array}$ & $\begin{array}{c}\text { Pas } \\
\text { du } \\
\text { tout }\end{array}$ \\
\hline
\end{tabular}

1) Au cours des 12 derniers mois, avez-vous toussé ?

2) Au cours des 12 derniers mois, avez-vous craché ?

3) Au cours des 12 derniers mois, avez-vous été essoufflé(e)?

4) Au cours des 12 derniers mois, avez-vous eu des crises de sifflement dans la poitrine?

5) Au cours des 12 derniers mois, combien de fois avez-vous eu de crises graves?

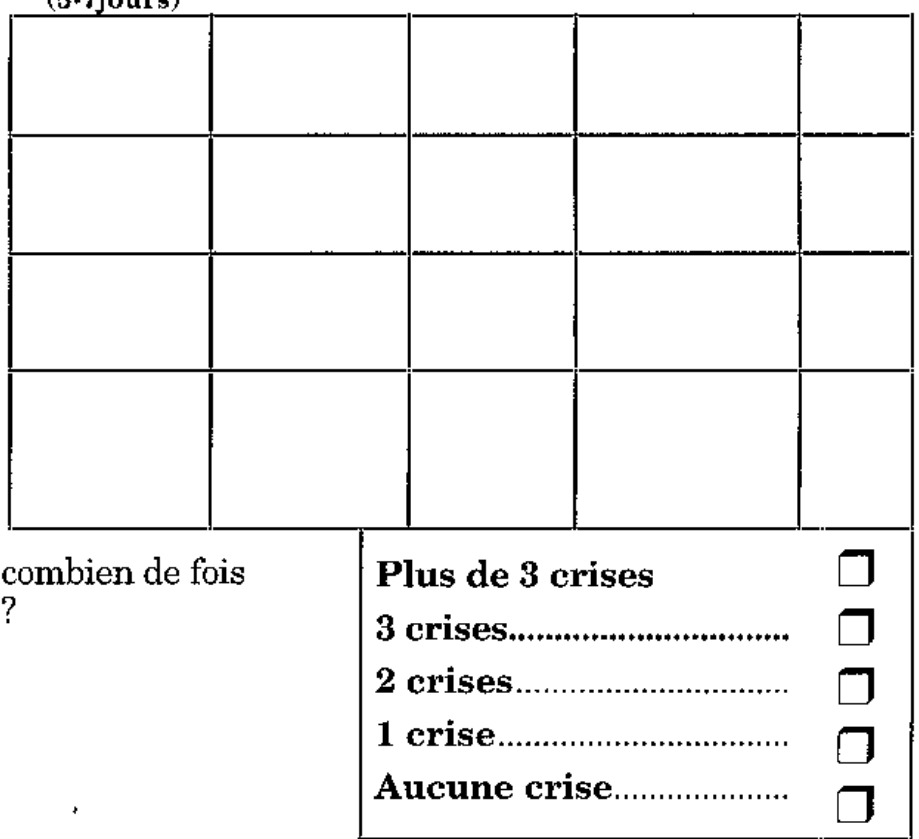

(passez à la question 7 si vous n'avez pas eu de crise grave)

6) Au cours des 12 derniers mois, combien de temps a duré la crise la plus pénible?

7) Au cours des 12 derniers mois, dans une semaine ordinaire, combien avez-vous eu de journées sans grand problème respiratoire?

8) Quand vous avez des sifflements, est-ce pire le matin?

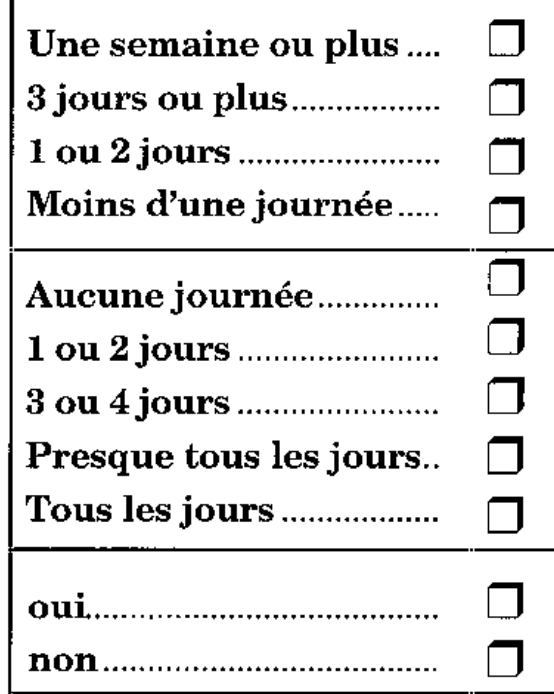




\section{2ème PARTIE}

\section{SECTION 1 Mettez une croix dans la case correspondant à votre réponse à chaque question.}

\section{QUE PENSEZ-VOUS DE VOTRE ETAT RESPIRATOIRE ?}

C'est mon plus gros problème.

Cela me pose pas mal de problèmes.

Cela me pose quelques problèmes.

Cela ne me pose aucun problème

\section{SI VOUS AVEZ OU SI VOUS AVEZ EU UNE ACTTVITE PROFESSIONNELLE}

Mes problèmes respiratoires m'ont obligé(e) à ne plus travailler.

Mes problèmes respiratoires m'empêchent parfois de travailler.

Mes problèmes respiratoires ne m'empêchent pas de travailler.

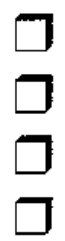

SECTION 2 VOICI QUELQUES SITUATIONS QUI, HABITUELLEMENT, VOUS ESSOUFFLENT.

Répondez en mettant une croix dans la case correspondant à votre situation ces jours-ci

Etre assis au repos

VRAI FAUX

Faire sa toilette ou s'habiller.

Marcher dans la maison.

Marcher à l'extérieur sur terrain plat.

Monter un étage.

Monter une côte.

Pratiquer une activité physique ou sportive

$\begin{array}{ll}\square & \square \\ \square & \square \\ \square & \square \\ \square & \square\end{array}$


SECTION 3 VOICI ENCORE QUELQUES SITUATIONS CONCERNANT VOTRE TOUX ET VOTRE ESSOUFFLEMENT.

Voulez-vous signaler celles qui correspondent à votre état ces jours-ci?

Ca me fait mal quand je tousse VRAI FAUX

Ca me fatigue quand je tousse.

Je suis essoufflé quand je parle

Je suis essoufflé quand je me penche.

Ma toux ou ma respiration perturbe mon sommeil.

$\square \square$

Je m'épuise vite en faisant une activité quotidienne (par exemple :

toilette, habillement, ménage)......

\section{SECTION 4 VOICI D'AUTRES EFFETS QUE VOS PROBLÈMES} RESPIRATOIRES PEUVENT ENTRAÎNER CHEZ VOUS.

Voulez-vous signaler celles qui s'appliquent à vous ces jours-ci?

Devant les autres je me sens gêné de tousser ou d'être essoufflé.

VRAI FAUX

Mes problèmes respiratoires gênent ma famille, mes amis ou mon

voisinage.

J'ai peur ou je panique quand je n'arrive plus à respirer

Je sens que je ne peux pas contrôler ma respiration

Je pense que mon état respiratoire ne va pas s'améliorer

Je suis devenu un grand malade en raison de mon état respiratoire

L'exercice physique est dangereux pour moi......

Tout me demande un effort.

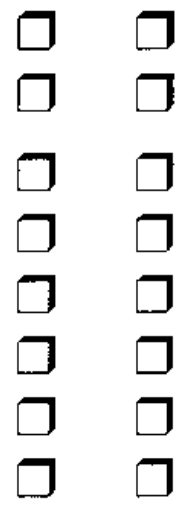

\section{SECTION 5 CETTE SECTION CONCERNE VOTRE TRAITEMENT (Médicaments, aérosols, oxygène, kinésithérapie...)}

Mon traitement ne m'aide pas beaucoup.

\section{VRAI FAUX}

Devant les autres, je me sens gêné de suivre mon traitement

Mon traitement a des effets désagréables chez moi.

Mon traitement me gêne beaucoup dans ma vie de tous les jours.

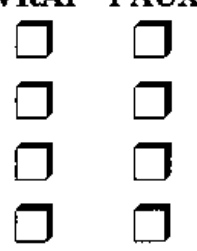




\section{SECTION 6 CETTE SECTION CONCERNE LES ACTIVITES QUOTIDIENNES QUI POURRAIENT ETRE GENEES PAR VOTRE RESPIRATION}

J'ai besoin de beaucoup de temps pour faire ma toilette ou pour m'habiller.

Je ne peux pas prendre un bain ou une douche, ou alors j'ai besoin de beaucoup de temps pour le faire...

VRAI FAUX

Je marche plus lentement que les autres ou je m'arrête pour me reposer

Des travaux comme le ménage me prennent beaucoup de temps ou je dois m'arrêter pour me reposer.

Si je monte un étage, je dois aller lentement ou m'arrêter.

Si je me dépêche ou si je marche vite, je dois m'arrêter ou ralentir.

Ma respiration rend pénible les activités telles que monter une côte, porter des objets en montant un étage, effectuer des travaux légers de jardinage, danser, jouer aux boules.

Ma respiration rend pénible les activités telles que porter des charges lourdes, bêcher le jardin, déblayer la neige, faire du jogging ou marcher rapidement, jouer au tennis, nager

Ma respiration rend pénible les activités telles que les travaux manuels lourds, la course à pied, le vélo, la natation rapide ou les sports de compétition.

$\square \square$
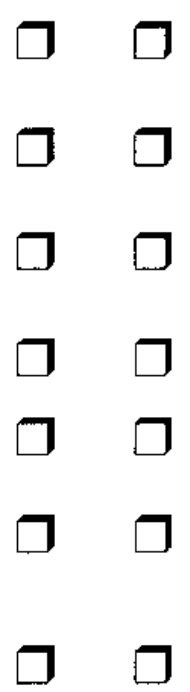

SECTION 7 DECRIVEZ AVEC QUELLE INTENSITE VOTRE ETAT RESPIRATOIRE RETENTIT DÕORDINAIRE SUR VOTRE VIE QUOTIDIENNE?

Je ne peux pratiquer aucun sport

VRAI FAUX

Je ne peux pas sortir pour me distraire ou me détendre

Je ne peux pas sortir faire les courses

Je ne peux pas faire le ménage ou bricoler.

Je ne peux pas m'éloigner beaucoup de mon lit ou de mon fauteuil.

$\begin{array}{ll}\square & \square \\ \square & \square \\ \square & \square \\ \square & \square\end{array}$


MAINTENANT, POURRIEZ-VOUS COCHER LA CASE CORRESPONDANT À CE QUI DÉCRIT LE MIEUX, SELON VOUS, LA MANIERE DONT VOTRE ETAT RESPIRATOIRE VOUS GENE

(Ne cochez qu'une seule case)

Cela ne m'empêche pas de faire ce que je veux

Cela m'empêche de faire une ou deux choses que j'aurais envie de faire.... $\square$

Cela m'empêche de faire la plupart des choses que j'aurais envie de faire. $\square$

Cela m'empêche de faire tout ce que j'aurais envie de faire.

Merci d'avoir rempli ce questionnaire.

Avant de terminer, veuillez vérifier que vous avez bien répondu à toutes les questions. 
ADL

\section{BPCO et Activités quotidiennes}

Votre médecin vous propose de remplir ce questionnaire car la mesure de votre souffle a montré des anomalies qui correspondent à une maladie respiratoire chronique qu'on appelle BPCO (dont vous avez peut-être entendu parler sous le nom de bronchite chronique). Ces problèmes de souffle peuvent vous gêner dans votre vie de tous les jours. Ce questionnaire permettra à votre médecin de vous proposer un meilleur suivi.

Pour répondre à ce questionnaire vous penserez à vos différentes activités quotidiennes et comment vous les effectuez habituellement.

1. Vous arrive-t-il d'être gêné(e) pour parler, discuter ?
$\square_{0}$
$\square_{1}$
$\square_{2}$
$\square_{3}$
Jamais
Parfois
Souvent
Tout le temps

2. Vous arrive-t-il d'être gêné(e) pour faire votre toilette, vous habiller ?
$\square_{0}$
$\square_{1}$
$\square_{2}$
$\square_{3}$
Jamais
Parfois
Souvent
Tout le temps

3. Vous arrive-t-il d'être gêné(e) pour faire vos courses dans votre quartier ?

\begin{tabular}{|c|c|c|c|c|c|}
\hline $\begin{array}{c}\square_{0} \\
\text { Jamais }\end{array}$ & $\begin{array}{c}\square_{1} \\
\text { Parfois }\end{array}$ & $\begin{array}{c}\square_{2} \\
\text { Souvent }\end{array}$ & $\begin{array}{c}\quad \square_{3} \\
\text { Tout le temps }\end{array}$ & $\begin{array}{c}\qquad \square_{4} \\
\text { Je ne peux } \\
\text { plus les faire }\end{array}$ & $\begin{array}{c}\square_{9} \\
\text { Ce n'est pas moi } \\
\text { qui m'occupe des }\end{array}$ \\
\hline
\end{tabular}

4. Vous arrive-t-il d'être gêné(e) pour faire le ménage ou du bricolage dans la maison ?

\begin{tabular}{|c|c|c|c|c|c|}
\hline $\begin{array}{c}\square_{0} \\
\text { Jamais }\end{array}$ & $\begin{array}{c}\square_{1} \\
\text { Parfois }\end{array}$ & $\begin{array}{c}\square_{2} \\
\text { Souvent }\end{array}$ & $\begin{array}{c}\quad \square_{3} \\
\text { Tout le temps }\end{array}$ & $\begin{array}{l}\qquad \square_{4} \\
\text { Je ne peux } \\
\text { plus le faire }\end{array}$ & $\begin{array}{c}\square_{9} \\
\text { Je ne fais ni } \\
\text { ménage, ni } \\
\text { bricolage }\end{array}$ \\
\hline
\end{tabular}

5. Vous arrive-t-il d'être gêné(e) au cours de vos déplacements à pied ?
$\square_{0}$
Jamais
$\square_{1}$
$\square_{2}$
$\square_{3}$
$\square_{4}$
Je ne peux plus le faire

6. Vous arrive-t-il d'être gêné(e) pour monter les escaliers ?
$\square_{0}$
$\square_{1}$
$\square_{2}$
$\square_{3}$
$\square_{4}$
Jamais Parfois Souvent Tout le temps Je ne peux

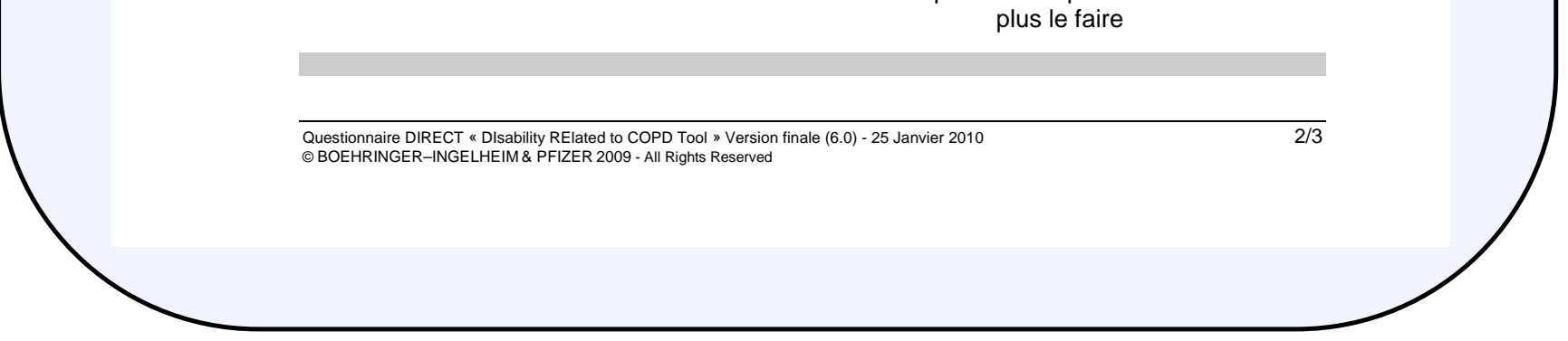


7. Vos difficultés respiratoires vous limitent-elles dans ce que vous aimez faire dans votre vie de tous les jours?
$\square_{0}$
$\square_{1}$
$\square_{2}$
Jamais
Parfois
Souvent
$\square_{3}$
Tout le temps

8. Vos difficultés respiratoires vous limitent-elles dans vos relations avec les autres (dans votre vie de tous les jours ou votre vie professionnelle)?
$\square_{0}$
$\square_{1}$
$\square_{2}$
$\square_{3}$
Jamais Parfois Souvent Tout le temps

\section{Vos difficultés respiratoires vous obligent-elles à compter sur les autres pour faire} certaines tâches?
$\square_{0}$
$\square_{1}$
$\square_{2}$
$\square_{3}$
Jamais Parfois Souvent Tout le temps

10. Vos difficultés respiratoires vous obligent-elles à vous reposer pendant la journée?
$\square_{0}$
$\square_{1}$
$\square_{2}$
$\square_{3}$
Jamais Parfois
Souvent
Tout le temps

11. Vos difficultés respiratoires vous limitent-elles dans vos relations intimes, votre vie sexuelle?

$\begin{array}{cccccc}\square_{0} & \square_{1} & \square_{2} & \square_{3} & \square_{4} & \begin{array}{c}\square_{9} \\ \text { Jamais }\end{array} \\ \text { Parfois } & \text { Souvent } & \text { Tout le temps } & \begin{array}{c}\text { Je ne peux } \\ \text { plus en avoir }\end{array} & \begin{array}{c}\text { Je n'ai pas de } \\ \text { relations sexuelles }\end{array}\end{array}$

12. Vos difficultés respiratoires sont-elles un handicap pour vous au quotidien?
$\square_{0}$
$\square_{1}$
$\square_{2}$
$\square_{3}$
Jamais
Parfois
Souvent
Tout le temps

\section{MERCI DE VOTRE PARTICIPATION}




\section{Cough And Sputum Assessment Questionnaire (CASA-Q)}

Ce questionnaire a été conçu pour nous permettre de mieux comprendre comment la toux et les glaires affectent votre vie de tous les jours.

Nous vous remercions de lire chaque question attentivement.

Répondez aux questions du mieux que vous pouvez, sans vous faire aider, et choisissez la réponse en cochant d'une croix $囚$ la case qui correspond le mieux à votre cas.

Il n'y a pas de réponse "juste" ou "fausse".

Toutes les informations que vous nous fournirez resteront confidentielles.

Le temps de remplissage de ce questionnaire est de 10 minutes environ. 


\section{CASA - Q}

\section{TOUX}

Les questions suivantes concernent votre toux. Nous vous remercions d'essayer de penser uniquement à votre toux quand vous répondez à ces questions.

1. Durant les 7 derniers jours, avez-vous toussé le matin au lever ?

\begin{tabular}{|c|c|c|c|c|}
\hline Pas du tout & Un petit peu & Moyennement & Beaucoup & Énormément \\
\hline$\square_{1}$ & $\square_{2}$ & $\square_{3}$ & $\square_{4}$ & $\square_{5}$ \\
\hline
\end{tabular}

2. Durant les 7 derniers jours, avez-vous toussé dans la journée ?

\begin{tabular}{|c|c|c|c|c|}
\hline Jamais & Rarement & $\begin{array}{c}\text { De temps en } \\
\text { temps }\end{array}$ & Souvent & Toujours \\
\hline$\square_{1}$ & $\square_{2}$ & $\square_{3}$ & $\square_{4}$ & $\square_{5}$ \\
\hline
\end{tabular}

3. Durant les 7 derniers jours, avez-vous eu des quintes de toux ?

\begin{tabular}{|c|c|c|c|c|}
\hline Jamais & Rarement & $\begin{array}{c}\text { De temps en } \\
\text { temps }\end{array}$ & Souvent & Toujours \\
\hline$\square_{1}$ & $\square_{2}$ & $\square_{3}$ & $\square_{4}$ & $\square_{5}$ \\
\hline
\end{tabular}

4. Durant les 7 derniers jours, avez-vous été fatigué(e) après avoir toussé ?

\begin{tabular}{|c|c|c|c|c|}
\hline Jamais & Rarement & $\begin{array}{c}\text { De temps en } \\
\text { temps }\end{array}$ & Souvent & Toujours \\
\hline$\square_{1}$ & $\square_{2}$ & $\square_{3}$ & $\square_{4}$ & $\square_{5}$ \\
\hline
\end{tabular}

5. Durant les 7 derniers jours, avez-vous été essoufflé(e) après avoir toussé ?

\begin{tabular}{|c|c|c|c|c|}
\hline Jamais & Rarement & $\begin{array}{c}\text { De temps en } \\
\text { temps }\end{array}$ & Souvent & Toujours \\
\hline$\square_{1}$ & $\square_{2}$ & $\square_{3}$ & $\square_{4}$ & $\square_{5}$ \\
\hline
\end{tabular}




\section{CASA - Q}

\section{TOUX}

6. Durant les 7 derniers jours, avez-vous été vous-même gêné(e) par votre toux?

\begin{tabular}{|c|c|c|c|c|}
\hline Pas du tout & Un petit peu & Moyennement & Beaucoup & Énormément \\
\hline$\square_{1}$ & $\square_{2}$ & $\square_{3}$ & $\square_{4}$ & $\square_{5}$ \\
\hline
\end{tabular}

7. Durant les 7 derniers jours, avez-vous limité vos sorties dans des lieux publics (bus, train, magasins, cinéma, restaurant, ...) à cause de votre toux ?

\begin{tabular}{|c|c|c|c|c|}
\hline Jamais & Rarement & $\begin{array}{c}\text { De temps en } \\
\text { temps }\end{array}$ & Souvent & Toujours \\
\hline$\square_{1}$ & $\square_{2}$ & $\square_{3}$ & $\square_{4}$ & $\square_{5}$ \\
\hline
\end{tabular}

8. Durant les 7 derniers jours, avez-vous dû interrompre par moments vos activités habituelles (conduire, faire le ménage ou la cuisine, faire des petits travaux dans la maison et autour de la maison, faire les courses...) à cause de votre toux ?

\begin{tabular}{|c|c|c|c|c|}
\hline Jamais & Rarement & $\begin{array}{c}\text { De temps en } \\
\text { temps }\end{array}$ & Souvent & Toujours \\
\hline$\square_{1}$ & $\square_{2}$ & $\square_{3}$ & $\square_{4}$ & $\square_{5}$ \\
\hline
\end{tabular}

9. Durant les 7 derniers jours, avez-vous dû interrompre par moments des conversations avec d'autres personnes à cause de votre toux ?

\begin{tabular}{|c|c|c|c|c|}
\hline Jamais & Rarement & $\begin{array}{c}\text { De temps en } \\
\text { temps }\end{array}$ & Souvent & Toujours \\
\hline$\square_{1}$ & $\square_{2}$ & $\square_{3}$ & $\square_{4}$ & $\square_{5}$ \\
\hline
\end{tabular}


CASA - Q

TOUX

10. Durant les 7 derniers jours, votre toux vous a -t-elle réveillé ou empêché de vous endormir ou de vous rendormir?

\begin{tabular}{|c|c|c|c|c|}
\hline Jamais & Rarement & $\begin{array}{c}\text { De temps en } \\
\text { temps }\end{array}$ & Souvent & Toujours \\
\hline$\square_{1}$ & $\square_{2}$ & $\square_{3}$ & $\square_{4}$ & $\square_{5}$ \\
\hline
\end{tabular}

11. Durant les 7 derniers jours, avez-vous été mal à l'aise à l'idée de gêner les gens en toussant?

\begin{tabular}{|c|c|c|c|c|}
\hline Jamais & Rarement & $\begin{array}{c}\text { De temps en } \\
\text { temps }\end{array}$ & Souvent & Toujours \\
\hline$\square_{1}$ & $\square_{2}$ & $\square_{3}$ & $\square_{4}$ & $\square_{5}$ \\
\hline
\end{tabular}

\section{GLAIRES}

Les questions suivantes concernent vos glaires. Nous vous remercions d'essayer de penser uniquement à vos glaires quand vous répondez à ces questions.

12. Durant les 7 derniers jours, quelle a été la consistance de vos glaires ?

\begin{tabular}{|c|c|c|c|c|}
\hline $\begin{array}{c}\text { Pas du tout } \\
\text { épaisse }\end{array}$ & Peu épaisse & $\begin{array}{c}\text { De temps en } \\
\text { temps }\end{array}$ & Assez épaisse & $\begin{array}{c}\text { Extrêmement } \\
\text { épaisse }\end{array}$ \\
\hline$\square_{1}$ & $\square_{2}$ & $\square_{3}$ & $\square_{4}$ & $\square_{5}$ \\
\hline
\end{tabular}

13. Durant les 7 derniers jours, avez-vous eu des glaires ?

\begin{tabular}{|c|c|c|c|c|}
\hline Jamais & Rarement & $\begin{array}{c}\text { De temps en } \\
\text { temps }\end{array}$ & Souvent & Toujours \\
\hline$\square_{1}$ & $\square_{2}$ & $\square_{3}$ & $\square_{4}$ & $\square_{5}$ \\
\hline
\end{tabular}




\section{CASA - Q}

\section{GLAIRES}

14. Durant les 7 derniers jours, avez-vous eu des difficultés pour respirer à cause de vos glaires?

\begin{tabular}{|c|c|c|c|c|}
\hline Jamais & Rarement & $\begin{array}{c}\text { De temps en } \\
\text { temps }\end{array}$ & Souvent & Toujours \\
\hline$\square_{1}$ & $\square_{2}$ & $\square_{3}$ & $\square_{4}$ & $\square_{5}$ \\
\hline
\end{tabular}

15. Durant les 7 derniers jours, avez-vous eu des difficultés pour faire remonter les glaires dans votre gorge?

\begin{tabular}{|c|c|c|c|c|}
\hline Pas du tout & Un petit peu & Moyennement & Beaucoup & Énormément \\
\hline$\square_{1}$ & $\square_{2}$ & $\square_{3}$ & $\square_{4}$ & $\square_{5}$ \\
\hline
\end{tabular}

16. Durant les 7 derniers jours, avez-vous été mal à l'aise à l'idée de gêner les gens quand vous avez eu des remontées de glaires?

\begin{tabular}{|c|c|c|c|c|}
\hline Jamais & Rarement & $\begin{array}{c}\text { De temps en } \\
\text { temps }\end{array}$ & Souvent & Toujours \\
\hline$\square_{1}$ & $\square_{2}$ & $\square_{3}$ & $\square_{4}$ & $\square_{5}$ \\
\hline
\end{tabular}

17. Durant les 7 derniers jours, avez-vous été vous-même gêné(e) par vos glaires ?

\begin{tabular}{|c|c|c|c|c|}
\hline Pas du tout & Un petit peu & Moyennement & Beaucoup & Énormément \\
\hline$\square_{1}$ & $\square_{2}$ & $\square_{3}$ & $\square_{4}$ & $\square_{5}$ \\
\hline
\end{tabular}

18. Durant les 7 derniers jours, est-ce que vos glaires vous ont gêné pour parler ?

\begin{tabular}{|c|c|c|c|c|}
\hline Jamais & Rarement & $\begin{array}{c}\text { De temps en } \\
\text { temps }\end{array}$ & Souvent & Toujours \\
\hline$\square_{1}$ & $\square_{2}$ & $\square_{3}$ & $\square_{4}$ & $\square_{5}$ \\
\hline
\end{tabular}


CASA - Q

\section{GLAIRES}

19. Durant les 7 derniers jours, avez-vous limité vos sorties dans des lieux publics (bus, train, magasins, cinéma, restaurant, ...) à cause de vos glaires ?

\begin{tabular}{|c|c|c|c|c|}
\hline Jamais & Rarement & $\begin{array}{c}\text { De temps en } \\
\text { temps }\end{array}$ & Souvent & Toujours \\
\hline$\square_{1}$ & $\square_{2}$ & $\square_{3}$ & $\square_{4}$ & $\square_{5}$ \\
\hline
\end{tabular}

20. Durant les 7 derniers jours, avez-vous dû interrompre par moments vos activités habituelles (conduire, faire le ménage ou la cuisine, faire des petits travaux dans la maison et autour de la maison, faire les courses...) pour vous débarrasser de vos glaires ?

\begin{tabular}{|c|c|c|c|c|}
\hline Jamais & Rarement & $\begin{array}{c}\text { De temps en } \\
\text { temps }\end{array}$ & Souvent & Toujours \\
\hline$\square_{1}$ & $\square_{2}$ & $\square_{3}$ & $\square_{4}$ & $\square_{5}$ \\
\hline
\end{tabular}

Nous vous remercions de votre participation 


\section{VSRQ}

\section{VISITE 1}

\section{ÉVALUATION DE LA QUALITÉ DE VIE Questionnaire VSRQ}

Madame, Mademoiselle, Monsieur,

Ce questionnaire a été conçu pour connaître les difficultés que vous pouvez avoir au quotidien à cause de votre essoufflement et les conséquences que cela a sur votre vie de tous les jours.

\section{Comment répondre au questionnaire?}

- Nous vous remercions de remplir ce questionnaire dans un endroit calme et si possible seul(e).

- Pour chacun des 8 items présentés ci-dessous, veuillez entourer le chiffre qui correspond à votre situation actuelle entre « 0 » pour une limitation extrême et « 10 » pour l'absence complète de limitation.

Nous vous remercions de votre participation

. Essoufflement :

$$
\text { |_0_|_1_||_2_|_3_||_4_|_5_||_6_|_7_||_8_|_9_||_10_| }
$$

. Activités de la vie quotidienne:

|_0_|_1_||_2_|_3_||_4_|_5_||6_|_7_||_8_|_9_||_10_|

Vie sociale :

|_0_|_1_||_2__3_||_4_|_5_||_6_|_7_||8_|_9_||_10_|

Qualité du sommeil :

|_0_|_1_||_2_|_3_||_4_|_5_||_6_|_7_||_8_|_9_||_10_|

. Plaisir :

|_0_|_1_||_2_|_3_||_4_|_5_||_6_|_7_||_8_|_9_||_10_|

. Energie :

|_0_|_1_||_2_|_3_||_4_|_5_||_6_|_7_||_8_|_9_||_10_|

. Inquiétude :

|_0_|_1_||_2_|_3_||_4_|_5_||_6_|_7_||_8_|_9_||_10_|

. Activité sexuelle :

|_0_|_1_||_2_|_3_||_4_|_5_||_6_|_7_||_8_|_9_||_10_|

TOTAL : 


\section{ECHELLE D'EPWORTH POUR L'ÉVALUATION DE LA SOMNOLENCE}

Dans les 8 circonstances suivantes, avez-vous un risque de vous endormir dans la journée?

- Si ce risque est inexistant : cochez 0

- Si ce risque est minime $\quad$ : cochez 1

- Si ce risque est modéré $\quad$ : cochez 2

- Si ce risque est important $\quad$ : cochez 3

\section{Exemple:}

Si le risque de vous endormir « assis en lisant un livre ou le journal » est modéré : cochez 2

1) Assis en lisant un livre ou le journal

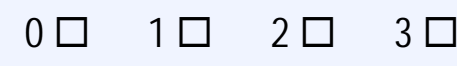

2) En regardant la télévision

3) Assis, inactif, dans un lieu public

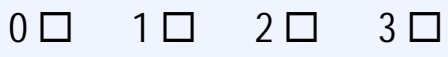
(cinéma, théâtre, salle d'attente)

4) Si vous êtes passager d'une voiture pour un trajet d'une heure

5) En étant allongé après le repas de midi

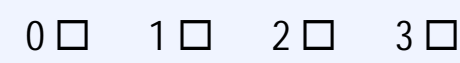

6) En étant assis, en parlant avec quelqu'un

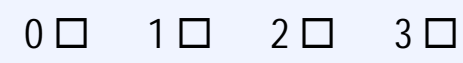

7) En étant assis, après un repas sans boisson alcoolisée

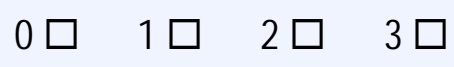

8) En étant au volant de la voiture, au cours d'un arrêt

$0 \square \quad 1 \square \quad 2 \square \quad 3 \square$
de la circulation de quelques minutes

TOTAL : 


\section{HOPITAL}

\section{SERVICE}

\section{FICHE BPCO}

\section{AUTOQUESTIONNAIRE}

\section{(JOINDRE LES RESULTATS D'EFR)}

NOM, prénom :

Date :

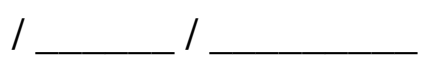

Remettre à votre médecin avec les questionnaires duement rempli Tél : 
Date : / l

Quelle est votre taille, en $\mathrm{cm}$ ?

Votre poids, en $\mathrm{kg}$ ?

Si vous êtes né dans un pays autre que la France, le noter ici :

Avez-vous eu au cours de votre vie une des maladies suivantes :

- asthme

- rhume des foins

- eczéma $\square$ Oui

$\square$ Oui

$\square$ Oui $\square$ Non

$\square$ Non

$\square$ Non

Avez-vous exercé une profession vous exposant à des fumées, gaz, toxiques, poussières ?

$\square$ Oui

$\square$ Non

Si oui, laquelle?

Entre quelle année et quelle année?

Quelle est la profession que vous avez effectué le plus longtemps (si différente de la précédente le cas échéant)?

Fumez-vous actuellement?

$\square$ Oui

$\square$ Non

Si oui, année de début

Avez-vous fumé auparavant ?

$\square$ Oui

Non

Si oui, entre quelle année et quelle année ?

En moyenne, combien de cigarettes par jour?

$\mathrm{CHU}$ : 

1

Nom : les $3 \overline{1 \text { ères lettres }}$

Prénom : les 2 1ères lettres

Combien de bronchites avez-vous eues l'année passée ?

Combien de fois, au cours de l'année écoulée, avez-vous du, à cause d'une bronchite :

- Prendre un antibiotique

- Prendre de la cortisone

- Consulter en urgence

- Etre hospitalisé

- Etre hospitalisé en réanimation

Toussez-vous régulièrement (en particulier le matin),

$\square$ Oui

$\square$ Non

Si oui, depuis combien de temps?

Avec parfois des crachats ?

Oui

$\square$ Non

Si oui, depuis combien de temps ?

Etes vous essoufflé lorsque vous faites des efforts?

Oui Non

Si oui, depuis combien de temps?

Souffrez-vous d'une des maladies suivantes:

- Insuffisance cardiaque

$\square$ Oui

$\square$ Non

- Arythmie

$\square$ Oui

$\square$ Non

- Angine de poitrine

$\square$ Oui

$\square$ Non

- Hypertension artérielle

$\square$ Oui

$\square$ Non

- Reflux gastro-oesophagien

$\square$ Oui

$\square$ Non

- Cancer du poumon

$\square$ Oui

$\square$ Non

- Diabète

$\square$ Oui

$\square$ Non

- Apnées du sommeil

$\square$ Oui

$\square$ Non

- Arthrose ou autre rhumatisme invalidant

$\square$ Oui

$\square$ Non

- Ostéoporose

$\square$ Oui

$\square$ Non

- Excès de cholestérol

$\square$ Oui

$\square$ Non

- Polyarthrite rhumatoïde

$\square$ Oui

$\square$ Non

$\mathrm{CHU}$ : 
Quels traitements prenez-vous actuellement de façon régulière ?

- En comprimés

- Par inhalation

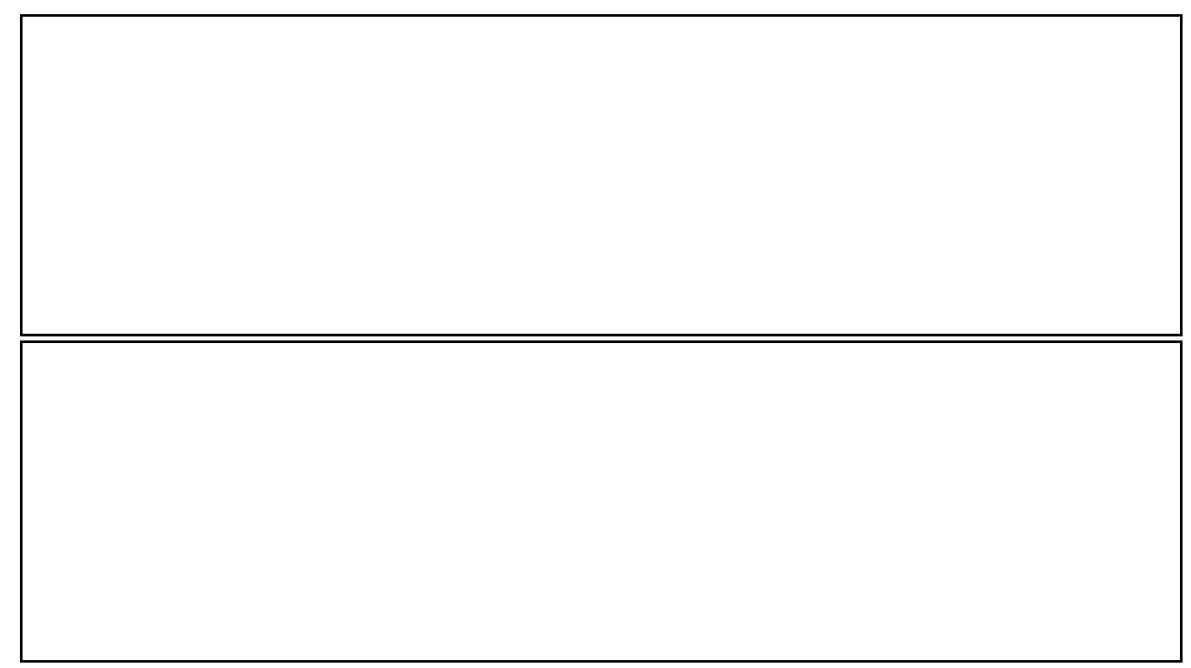

Etes vous vacciné contre la grippe ?

$\square$ Oui

$\square$ Non

Contre le pneumocoque?

$\square$ Oui

Non

Avez-vous de manière habituelle ou répétée :

Le nez bouché

Le nez qui coule

Des éternuements

Une perte d'odorat $\square$ Oui

$\square$ Oui

$\square$ Oui

$\square$ Oui $\square$ Non

$\square$ Non

Non

$\square$ Non

Si oui à une des questions précédentes, le ou les symptômes sont-ils rythmés par les saisons

$\square$ Oui

$\square$ Non

EFR

Résultats ci-joint

Date de l'examen:

$\mathrm{CHU}$ : 
Annexe 2 


\section{Questionnaire Dyspnée BPCO}

Chers Collègues,

Dans le cadre du travail de thèse d'exercice d'Hortense Carette, interne de Pneumologie à Nancy, nous nous intéressons à la prise en charge de la dyspnée chez les patients atteints de BPCO.

Ce questionnaire a pour objectif d'apprécier les pratiques actuelles de prise en charge de la dyspnée chez les patients atteints de BPCO tous stades confondus, par les Pneumologues de Lorraine, quelque soit le mode d'exercice.

Nous vous serions reconnaissants de prendre quelques minutes pour répondre à ce questionnaire dont les données seront exploitées anonymement.

Temps estimé : 5 minutes

A noter :

Nous considérons comme traitement de la dyspnée, toute intervention destinée à traiter spécifiquement le symptôme en dehors des traitements "physiopathologiques" des mécanismes en cause. Par exemple, nous considérons l'utilisation d'un bronchodilatateur inhalé chez un patient présentant une dyspnée en rapport avec une exacerbation de BPCO comme un traitement "physiopathologique" et non comme un traitement symptomatique de la dyspnée.

\section{*Obligatoire}

\section{Adresse e-mail *}

\section{Q0 - Avant de débuter ce sondage, nous souhaiterions relever quelques données démographiques:}

Les données de ce questionnaire sont exploitées de manière anonyme.

\section{Age : *}

Une seule réponse possible.

$$
\begin{aligned}
& <30 \text { ans } \\
& 30-40 \text { ans } \\
& 40-50 \text { ans } \\
& 50-60 \text { ans } \\
& >60 \text { ans }
\end{aligned}
$$

\section{Sexe : *}

Une seule réponse possible.

Homme

Femme 


\section{Ancienneté d'exercice :}

Une seule réponse possible.
$<5$ ans
5-10 ans
10-20 ans
$>20$ ans

\section{Lieu d'exercice : *}

Plusieurs réponses possibles.

Libéral

CHU

$\square \mathrm{CHR}$

Etablissement Privé

Exercice Mixte

6. Spécificité d'activité : *

Plusieurs réponses possibles.

BPCO

Fibrose Pulmonaire

Oncologie

HTAP

Pathologie Neuromusculaire

Soins palliatifs 
7. Q1 - Parmi les interventions thérapeutiques suivantes non pharmacologiques, lesquelles prescrivez/conseillez-vous pour soulager de façon symptomatique la dyspnée? *

Plusieurs réponses possibles.

Sophrologie

Relaxation

Yoga

Thérapie de Pleine Conscience (= Mindfulness ou MBSR)

Acupuncture

Hypnose Médicale

Distraction auditive

Prise en charge par un psychotérapeute

$\square$ Vibration au niveau de la paroi thoracique

Pratique sportive

Réhabilitation respiratoire

Réentrainement musculaire inspiratoire

Aucun

Autre :

8. Q2 - A quelle fréquence prescrivez-vous ces traitements (non pharmacologiques) dans le but spécifique de soulager la dyspnée? *

Une seule réponse possible.

Souvent

Parfois

Rarement

Jamais

9. Q3 - Chez les patients ambulatoires, parmi les traitements suivants, lesquels prescrivez-vous pour soulager la dyspnée? *

Plusieurs réponses possibles.

Non Concerné

Opioïdes par voie systémique

Opioïdes en nébulisation

Benzodiazépines

Oxygène (en l'absence d'hypoxémie sévère)

Furosémide Inhalé

Diurétiques (hors Insuffisance Cardiaque)

$\square$ Aucun

Autre : 
10. Q4 - Chez les patients hospitalisés, parmi les traitements suivants, lesquels prescrivez-vous pour soulager la dyspnée? *

Plusieurs réponses possibles.

\section{Non Concerné}

Opioïdes par voie systémique

Opioïdes en nébulisation

Benzodiazépines

Oxygène (en l'absence d'hypoxémie sévère)

Furosémide Inhalé

$\square$ Diurétiques (hors Insuffisance Cardiaque)

Autre :

11. Q5 - Chez les patients en fin de vie en soins palliatifs, parmi les traitements suivants, lesquels prescrivez-vous pour soulager la dyspnée? *

Plusieurs réponses possibles.

\section{Non Concerné}

Opioïdes par voie systémique

Opioïdes en nébulisation

Benzodiazépines

Oxygène (en l'absence d'hypoxémie sévère)

Furosémide Inhalé

$\square$ Diurétiques (hors Insuffisance Cardiaque)

$\square$ Autre :

12. Q6 - A quelle fréquence prescrivez-vous des traitements médicamenteux dans le but spécifique de soulager la dyspnée? (C'est à dire : des substances pharmacologiques qui agissent directement sur la perception de ce symptôme et qui ne sont pas destinées à améliorer la physiopathologie sous-jacente). * Une seule réponse possible.

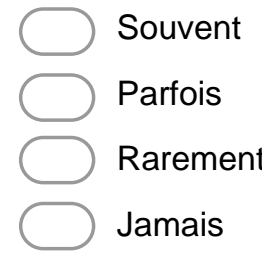

13. Q7 - Votre prise en charge de la dyspnée dépend-elle de la pathologie sous jacente? *

Une seule réponse possible.

Oui

Non 
14. Si oui, précisez :

15. Q8 - Etes-vous réticent à prescrire des Opioïdes systémiques pour soulager la dyspnée? *

Une seule réponse possible.

Oui

Non

16. Si Oui, veuillez indiquer les raisons de votre réticence :

Plusieurs réponses possibles.

Risque d'addiction

Risque de développer une tolérance aux opioïdes

$\square$ Risque de dépression respiratoire

$\square$ Risque de constipation/nausées/vomissements

$\square$ Risque de réduction de la durée de vie

Risque de somnolence

Absence d'AMM

Absence d'efficacité

Absence de recommandation concernant les doses

Autre :

17. Q9 - Etes-vous réticent à prescrire des Benzodiazépines pour soulager la dyspnée? Une seule réponse possible.

Oui

$\bigcirc$ Non 
18. Si Oui, veuillez indiquer les raisons de votre réticence :

Plusieurs réponses possibles.

Risque d'addiction

Risque de développer une tolérance aux benzodiazépines

Risque de dépression respiratoire

Risque de réduction de la durée de vie

Risque de somnolence

Absence d'AMM

$\square$ Absence d'efficacité

$\square$ Absence de recommandation concernant les doses

$\square$ Autre :

19. Q10 - Si vous n'avez JAMAIS prescrit de traitement pour soulager la dyspnée, veuillez en indiquer les raisons.

Plusieurs réponses possibles.

II n'y a aucun traitement efficace

II y a trop de risque d'effets secondaires

En raison du risque de dépression respiratoire

En raison du risque de somnolence

$\square$ En raison de risque d'addiction

En l'absence d'AMM

Car il existe d'autres interventions non médicamenteuses

$\square$ Autre :

20. Q11 - Pour vous, que recouvre le terme "dyspnée réfractaire" ? *

21. Q12 - Quel terme proposeriez-vous pour définir une dyspnée intense, chronique qui persiste après le traitement de la pathologie sous-jacente? * 



\section{RÉSUMÉ DE LA THÈSE}

Objectif : L'objectif principal de cette étude est la description du symptôme de dyspnée et de sa prise en charge chez les patients atteints de BPCO en Lorraine.

Méthodes : Notre étude est réalisée en deux parties. La première partie est une étude prospective descriptive via la cohorte iBPCO. La population étudiée concerne les 120 premiers patients inclus, au sein du CHU de Nancy du 01/06/15 au 25/02/16. La seconde partie est une étude déclarative multicentrique via un questionnaire réalisé par le groupe dyspnée de la SPLF, adapté aux patients BPCO, envoyé aux pneumologues de Lorraine. La dyspnée sévère est définie par un stade $m M R C \geq 3$. Parmi les patients dyspnéiques sévères, une dyspnée réfractaire est retenue si la dyspnée sévère persiste plus de 3 mois, malgré un traitement optimal de la pathologie sous-jacente, avec une intensité importante.

Résultats : Parmi les 120 patients inclus, 52 patients (43\%) sont obèses et 19 patients $(16 \%)$ ont une insuffisance cardiaque. Soixante-quatre patients $(53 \%)$ ont une dyspnée sévère, dont 41 patients (soit $64 \%$ d'entre eux) ont une dyspnée réfractaire. Concernant les comorbidités, il n'y a pas de différence significative entre le groupe dyspnée sévère et non sévère. Les patients qui ont une dyspnée sévère ont significativement plus de symptômes anxio-dépressifs (SAD) que les non sévères : score HAD anxiété positif $(>10) 34,4 \%$ versus $11,1 \%(p=0,003)$; score HAD dépression positif $(>10) 31,1 \%$ versus $7,4 \%(p=0,001)$. Parmi les 120 patients inclus, 41 patients $(34 \%)$ ont eu une réhabilitation respiratoire (RR). Concernant les patients avec une dyspnée sévère, $41 \%$ ont effectué une RR et $94 \%$ ont un traitement pharmacologique étiologique considéré optimal selon les recommandations de la SPLF. Les patients qui ont une dyspnée réfractaire ont eu une RR pour 41,5\% d'entre eux. Aucun patient n'a de traitement symptomatique par morphinique à visée eupnéisante. D'après le questionnaire, la prise en charge non pharmacologique de la dyspnée concerne essentiellement la RR et la pratique sportive. Les morphiniques à visée eupnéisante par voie systémique sont le traitement pharmacologique le plus déclaré, particulièrement en situation palliative à $91 \%$. Les jeunes médecins ( $<10$ ans d'expérience) semblent plus réticents que leurs aînés à la prescription de morphiniques dans ce contexte. Quarante pour cent des médecins réticents à l'utilisation de morphiniques le justifient par manque de recommandations.

Conclusion : La prise en charge de la dyspnée et des comorbidités fréquentes comme les SAD dans le cadre de la BPCO n'est pas optimale. Promouvoir la RR et les traitements pharmacologiques symptomatiques représentent un élément déterminant dans la prise en charge globale de la BPCO.

TITRE EN ANGLAIS: Management of dyspnea in COPD, real life study in Lorraine.

THÈSE : MÉDECINE SPÉCIALISÉE - ANNÉE 2017

MOTS CLES : BPCO ; dyspnée ; syndrome anxio-dépressif ; traitement.

INTITULÉ ET ADRESSE : UNIVERSITÉ DE LORRAINE

Faculté de Médecine de Nancy

9, avenue de la Forêt de Haye

54505 VANDOEUVRE LES NANCY Cedex 